\title{
Scattering amplitudes in the light-front formalism
}

\author{
C. Cruz-Santiago, ${ }^{1}$ P. Kotko, ${ }^{1}$ A.M. Staśto, ${ }^{1,2}$ \\ ${ }^{1}$ Department of Physics, Davey Laboratory, The Pennsylvania State University, \\ University Park, PA 16802, United States \\ ${ }^{2}$ H. Niewodniczański Institute of Nuclear Physics, Polish Academy of Sciences, \\ Kraków, Poland
}

July 22,2015

\begin{abstract}
We review the methods of the computation of the scattering amplitudes in the light-front formalism. Basics of the light-front quantization, as well as helicity methods and different types of recursion relations for scattering amplitudes are reviewed. Computation of the wave-functions and fragmentation functions within the light-front theory are presented. Methods for obtaining offshell scattering amplitudes are presented together with the new recursion relations which include the gauge invariant extensions of the off-shell amplitudes. Finally, relation between the formalism which makes use of the Wilson lines and light-front formalism is discussed.
\end{abstract}




\section{Contents}

1 Introduction $\quad 3$

2 Light-front formalism $\quad 6$

2.1 Light-front coordinates . . . . . . . . . . . . . . . . . . . . . . . 6

2.2 Poincaré group . . . . . . . . . . . . . . . . . . . . . . . . . . . . 7

2.3 Old-fashioned time ordered perturbation theory f . . . . . . . . . . . . 8

2.4 Light-front rules for the scalar theory . . . . . . . . . . . . . . . . . . . 12

2.5 Light-front formalism for the gauge theory . . . . . . . . . . . . . . . . 13

2.5.1 Gluon propagator in the light-front theory . . . . . . . . . . . . . . 13

2.5.2 Light-front quantization for QCD . . . . . . . . . . . . . . . . 14

2.5.3 Light-front rules for the QCD . . . . . . . . . . . . . . . . . . . 17

$\begin{array}{llr}3 & \text { Scattering amplitudes in the helicity formalism } & 18\end{array}$

3.1 Helicity formalism . . . . . . . . . . . . . . . . . . . . . . . 18

3.1 .1 Polarization vectors . . . . . . . . . . . . . . . . . . . . 20

3.1 .2 Color ordering . . . . . . . . . . . . . . . . . . . . . . 22

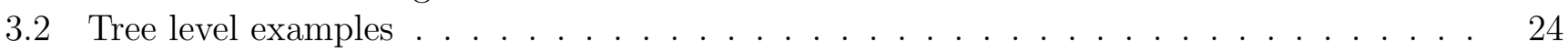

3.3 Berends-Giele recursion relations for off-shell currents . . . . . . . . . . . . . . . . 26

3.4 BCFW recursion relations for tree-level amplitudes . . . . . . . . . . . . . . . . 28

4 Wave functions and fragmentation functions on the light-front 31

4.1 Wave functions on the light-front . . . . . . . . . . . . . . . . . 32

4.2 Relation between spinor helicity formalism and light-front variables . . . . . . . . . 38

4.3 Factorization of the fragmentation functions . . . . . . . . . . . . . . . 39

4.4 Cluster decomposition . . . . . . . . . . . . . . . . . . . . . . 41

4.5 Fragmentation functions for simple helicity configurations . . . . . . . . . . . . . . . 43

5 Scattering amplitudes in the light-front formalism 44

5.1 From fragmentation functions to $2 \rightarrow n$ amplitudes $\ldots \ldots \ldots \ldots$

5.1.1 Vanishing of $\mathcal{M}(-+\cdots+)$ and $\mathcal{M}(++\cdots+)$ on the light front . . . . . . 51

5.2 Berends-Giele recursion relations in the light-front framework . . . . . . . . . . . 52

$5.3 \mathrm{MHV}$ amplitudes from light-front recursion . . . . . . . . . . . . . . . . 54

5.4 Wilson lines and gauge invariance of the off-shell amplitudes . . . . . . . . . . . 57

5.5 Relation of Wilson lines to the light-front ampliudes . . . . . . . . . . . . . . . . 60

5.6 The Ward identity for light-front amplitudes . . . . . . . . . . . . . . . . . . 62 


\section{Introduction}

One of the most important and fundamental questions in the field of elementary particle physics is to understand and unveil the details of the structure of hadrons, such as protons and neutrons, in terms of the quark and gluon degrees of freedom. Since several decades there have been quite a number of collider experiments which allowed to perform various tests of the particle content and interactions of the Standard Model, among them strong interactions. These experiments delivered a lot of important information about the hadron structure and hadron interactions in terms of quark and gluons degrees of freedom. Strong interactions which are responsible for the hadron structure are described by the nonabelian gauge theory of Quantum Chromodynamics (QCD). Perturbative calculations in QCD which involve processes at large transverse momenta have been extensively developed and the predictions have been tested in these high-energy collisions.

Nevertheless, Quantum Chromodynamics despite many tests and decades of progress in calculations, is still a theory which has not been completely understood and solved. Its non-abelian nature, leading to complex nonlinear interactions, many emergent phenomena like confinement and hadron spectra escape yet a complete understanding. One of the properties of QCD that is usually stressed is the complexity of the QCD vacuum, which in part is responsible for the emergent phenomena, like for example chiral symmetry breaking in QCD. It is therefore a legitimate question as to whether there exists another formulation of quantum field theory, in particular QCD, such that it would be more suitable for the description of the high energy phenomena alluded to above and would hopefully be simpler.

The light-front quantization potentially provides such a reformulation of the quantum field theory. The quantization procedure on the the light-front (or the null-plane) was first proposed a long time ago by Dirac [1] as an alternative approach to the more standard instant-time quantization. Dirac considered different forms for the relativistic dynamics: the instant form, the light-front form and the point form. As pointed out in this original work [1] one of the interesting features of the light-front quantization is the presence of only three dynamical Poincaré generators which describe the evolution of a system in light-front time, see also for example [2]. The 10 generators of the Poincaré group can be divided into kinematical, i.e. such that leave the quantization surface invariant and the dynamical ones, or the Hamiltonians which move this surface. Typically the latter are the more complicated ones. The standard instant form has 6 kinematical and 4 dynamical generators while the front-form has 7 kinematical and 3 dynamical ones. Thus, one may hope that the light-front formalism may lead to a simpler solution of problems in relativistic quantum mechanics than other quantization schemes which typically possess larger number of dynamical operators. The other advantage of the light-front form is the absence of the square root in the Hamiltonians, which can pose some mathematical difficulties in the instant time approach.

Another interesting feature of the light front formulation is the fact that the Poincare group in this case has a subgroup which exhibits algebraic structure isomorphic to the Galilean symmetry group of non-relativistic quantum mechanics in two dimensions $[3,4]$. There is two dimensional transverse momentum and the role of the mass is played by the longitudinal momentum fraction of the particles.

It has been also stated in many works, see for example [5-8], that the vacuum on the light-front is essentially structureless. In fact the arguments about simplicity of the vacuum have been provided first by the direct analysis of diagrams in the infinite momentum frame [9]. It has been shown, that in this limit the diagrams basically fall into two distinctive groups. The first one is the group with vacuum fluctuations, and it turns out that these diagrams vanish in the infinite momentum frame. As a result the only non-vanishing diagrams on the light-front, come from the second group where the particles in these diagrams all have positive $p^{+}$momenta. In the case of the theory with scalar particles this dramatically reduces the number of diagrams which are needed to be considered and eliminates vacuum diagrams, i.e. diagrams where the particles and antiparticles are created out of the vacuum (in the theory with spin degrees of freedom there are additional instantaneous interactions which add to the 
overall number of diagrams).

To be precise, the vacuum on the light-front is structureless up to zero modes, that is states where $p^{+} \rightarrow 0$ for which a special treatment may be necessary, like discrete quantization which isolates these modes. This has been investigated in much detail in [10]. It was also argued that the zero modes contribute to the Higgs vacuum expectation value in the Standard Model [11].

This simplicity of the light-front vacuum allows to define unambiguously the partonic content of hadrons. Due to the simplicity of the light-front vacuum the Fock expansion provides the complete relativistic basis for diagonalizing the theory. Another consequence of the structureless vacuum is that it may lead to a new insight into the understanding of the spontaneous breaking of the chiral symmetry in QCD, for a recent nice review see [12]. Recently, it has been argued that in the light-front formalism the quark condensates must have finite support within the hadrons. It is normally assumed that the vacuum quark condensate has a non-zero value due to the complexity of the QCD vacuum and this is responsible for the dynamical chiral symmetry breaking. However, the fact that such non-zero vacuum condensates exist, in principle poses a big problem for the light-front formulation where the ground state is a structureless Fock vacuum. It has thus been argued, see [13-16], that the quark condensates thus must have support only within the hadrons and as such the dynamical chiral symmetry is a property of the hadron wave function and not of the QCD vacuum itself [5].

The light-front framework has been used to investigate the hadron dynamics from AdS/CFT correspondence [17]. In the high-energy approximation, the light-front methods allowed to compute the soft gluon component of the heavy onium wave function and to obtain a correspondence with the hard Pomeron in QCD [18].

Thus the light-front formalism, thanks to the simpler vacuum structure and the isomorphism with the Galilean subgroup, can provide a unique insight into some convoluted aspects of QCD. It is therefore natural to pose the question as to whether it can offer some insight into the structure of the scattering amplitudes in QCD. Of particular interest are the computations of the scattering amplitudes with several number of external legs in perturbative Quantum Chromodynamics and for the specified helicity configurations. The non-vanishing amplitudes for the simplest configurations of these helicities are known as the Maximally Helicity Violating (MHV) amplitudes. These amplitudes can then be used to evaluate the cross section for the production of many particles in the final state, like the (multi)jet production, which has been extensively measured in the hadronic colliders and provides one of the best test of the QCD.

In the recent decades there has been an outstanding progress in the computation of the scattering amplitudes. Starting from the famous conjecture by Parke and Taylor [19], where the multi-gluon amplitudes with special helicity configurations have been argued to have a very simple form when expressed in terms of the spinor products. This conjecture was rigorously proved in the later works by use of the Berends-Giele recursion relations [20], which allowed for the computation of the helicity amplitudes with arbitrary number of external legs. Later on, new recursion relations have been discovered, the BrittoCachazo-Feng-Witten (BCFW) [21, 22] recursion relations which involve only on-shell amplitudes with shifted complex momenta. Presently, there exists a number of automated tools for the evaluation of the multi-parton amplitudes for different species of particles, see for example [23-28].

Light-front methods were used to compute the gluon wave functions and fragmentation functions $[29,30]$. Gluon wave functions are off-shell amplitudes which describe the transition from 1 to $n$ gluons, where the initial particle is on-shell and all the outgoing are off-shell. The fragmentation function is a similar transition but in this case the initial gluon is off-shell and the final particles are on-shell. In the case of the light-front theory they differ by the energy denominators which are kept non-zero in the final state (for gluon wave functions) or for the initial state (for the fragmentation functions). One can then formulate recursion relations for these objects on the light-front, and in some specific cases of helicity configurations these recursion relations can be solved exactly $[29,30]$. The fragmentation functions differ from the wave functions by the fact that the recursion relation utilizes the factorization 
property (otherwise known as a cluster decomposition [31]). It can be thus shown that this factorization property and the resulting recurrence relation for the fragmentation functions are light-front analogs of the Berends-Giele recursion relation [20].

Later on, [32] it was shown that the amplitudes can be obtained from the fragmentation functions or wave functions in a simple way. The recursion relations have been solved for off-shell amplitudes with one leg off-shell in the $(--+\cdots+)$ configurations. In the on-shell limit these amplitudes coincide with the well known Parke-Taylor amplitudes. The off-shell amplitude which solves the recursion relation has an interesting property in that it is equal to the sum of another off-shell amplitude which has the functional form identical to the MHV amplitude and the term which is proportional to the energy denominator and is a product of the amplitudes with lower number of legs.

This interesting relation has been thoroughly examined in the recent work [33]. It was demonstrated, that its origin stems from the gauge invariance properties of QCD. Namely, it was shown in [34] that, for any off-shell amplitude one can construct its gauge invariant extension, using matrix elements which involve straight infinite Wilson lines. As it turns out, those Wilson lines also encode certain recursion which turns out to be identical to the recurrence found in the light-front theory. Thus the new off-shell amplitude that appears in the light-front recursion, which has the functional form identical to MHV, is actually gauge invariant. To be precise, it satisfies the Ward identity with respect to the on-shell legs [33]. In addition, it was demonstrated that in order to satisfy Ward identities explicitly on the lightfront, one needs to reformulate the methods of the light-front perturbation theory. This stems from the fact that the minus-components of the momenta do appear in the numerators of the amplitudes in the light-front formulation when the Ward identity is computed. As a result, new instantaneous terms need to be taken into account for the Ward identity to hold and they restore the full momentum conservation in the vertices, for all light cone components.

The structure of this review is as follows. Next section begins with the introduction of the light-front formalism, the basic notation and the variables. We discuss the Poincaré group on the light-front and we point out the existence of the Galilean subgroup. Then, we remind the methods of the old-fashioned perturbation theory in the scalar case and how those rules need to be modified when going into the infinite momentum frame. We comment on the vanishing of the vacuum diagrams. At the end of this section we introduce the propagator in the gauge theory and discuss the light-front rules for QCD.

In Sec. 3 basic facts about the helicity formalism are discussed like the notation, spinor products, definition of the polarization vectors and color ordering. Next, simple examples are given for the lowest order helicity amplitudes and for the simplest cases of the helicity configurations, together with the Parke-Taylor amplitudes. Furthermore, Berends-Giele recursion relations for the off-shell currents in QCD are introduced and discussed. Sec. 3 is concluded with the brief discussion of the BCFW recursion for the tree-level on-shell amplitudes and some comments about its extension to the off-shell case. Sec. 4 begins with the explicit construction of the gluon-wave function on the light-front through the corresponding recurrence relations. Later on, the fragmentation functions are introduced and the factorization property is discussed together with the related property of the cluster decomposition. Explicit form for the simple helicity configuration for the fragmentation function is given. An interesting duality between the wave functions and the fragmentation functions is discussed at the end of this section. Finally, Sec. 5 is devoted to the derivation and the analysis of the properties of the off-shell amplitudes on the light-front. The section begins with the arguments for using the crossing properties on the light-front which enables to utilize the $1 \rightarrow n$ fragmentation functions for the computation of the $2 \rightarrow n$ scattering amplitudes. Next, recursion relations for the off-shell amplitudes on the light-front are discussed which are analogous to the Berends-Giele recursion relations. It is shown that they are solved by another recursion relation which involves an off-shell object which has the exact same functional form as the MHV amplitude. Later on, Wilson lines and the recurrence relations which involve them are introduced and they turn out to be identical to the recurrence relations on the light-front. This section ends with the discussion of the Ward identity on the light-front together with the explicit check 
of this identity for the case of the simplest amplitude.

\section{Light-front formalism}

\section{$2.1 \quad$ Light-front coordinates}

We begin with the definition of the variables that are used on the light front [35]. Let $x^{\mu}$ be a contravariant space-time coordinate four-vector,

$$
x^{\mu}=\left(x^{0}, x^{1}, x^{2}, x^{3}\right),
$$

with the metric tensor in the standard convention $g^{00}=1, g^{11}=g^{22}=g^{33}=-1$. One can then perform the transformation to a new coordinate basis and introduce a new time-like component, referred to as the light-front time,

$$
x^{+}=x^{0}+x^{3},
$$

and a corresponding space-like component

$$
x^{-}=x^{0}-x^{3} .
$$

Therefore in the new basis four-vectors are written as $x^{\mu}=\left(x^{+}, x^{1}, x^{2}, x^{-}\right)$and the scalar product of two four-vectors is defined as

$$
x \cdot p=x^{\mu} p_{\mu}=\frac{1}{2}\left(x^{+} p^{-}+x^{-} p^{+}\right)-\vec{x}_{\perp} \cdot \vec{p}_{\perp},
$$

where $x_{\perp}^{\mu}=\left(0, x^{1}, x^{2}, 0\right)=\left(0, \vec{x}_{\perp}, 0\right)$ and $\vec{x}_{\perp}=\left(x_{1}, x_{2}\right)$. In particular we have

$$
x^{2}=x^{+} x^{-}-\vec{x}_{\perp}^{2}
$$

The covariant four-vectors are obtained with the use of the metric tensor, which in the light-front basis is defined as

$$
g_{\mu \nu}=\left(\begin{array}{cccc}
0 & 0 & 0 & \frac{1}{2} \\
0 & -1 & 0 & 0 \\
0 & 0 & -1 & 0 \\
\frac{1}{2} & 0 & 0 & 0
\end{array}\right), \quad g^{\mu \nu}=\left(\begin{array}{cccc}
0 & 0 & 0 & 2 \\
0 & -1 & 0 & 0 \\
0 & 0 & -1 & 0 \\
2 & 0 & 0 & 0
\end{array}\right) .
$$

Note that, with the above definition of the metric tensor we have $x_{+}=\frac{1}{2} x^{-}, x_{-}=\frac{1}{2} x^{+}$. In general to change from the standard basis to the light-front basis one needs to perform the following transformation

$$
x^{\mu} \rightarrow L_{\nu}^{\mu} x^{\nu}, \quad A^{\mu \nu} \rightarrow L_{\alpha}^{\mu} A^{\alpha \beta} L_{\beta}^{\nu},
$$

with the transformation matrix

$$
L_{\nu}^{\mu}=\left(\begin{array}{cccc}
1 & 0 & 0 & 1 \\
0 & 1 & 0 & 0 \\
0 & 0 & 1 & 0 \\
1 & 0 & 0 & -1
\end{array}\right)
$$




\subsection{Poincaré group}

The standard generators of the infinitesimal Lorentz transformations form Poincaré algebra, for which the conventional relations hold in the standard basis

$$
\begin{aligned}
{\left[P_{\mu}, P_{\nu}\right] } & =0 \\
{\left[M_{\mu \nu}, P_{\rho}\right] } & =i\left(g_{\nu \rho} P_{\mu}-g_{\mu \rho} P_{\nu}\right) \\
{\left[M_{\mu \nu}, M_{\rho \sigma}\right] } & =i\left(g_{\mu \sigma} M_{\nu \rho}+g_{\nu \rho} M_{\mu \sigma}-g_{\mu \rho} M_{\nu \sigma}-g_{\nu \sigma} M_{\mu \rho}\right) .
\end{aligned}
$$

The generators for the boosts are $M_{i 0}=K_{i}$ and for the rotations $M_{i j}=\epsilon_{i j k} J_{k}$, where $i, j=1,2,3$ and $\epsilon_{i j k}$ is the antisymmetric tensor.

In the light-front basis the generators can be obtained by performing the transformation given by (7) and (8), in which case they have the following form

$$
P^{\mu}=\left(P^{+}, P^{1}, P^{2}, P^{-}\right)
$$

where

$$
P^{+}=P^{0}+P^{3}, \quad P^{-}=P^{0}-P^{3}, \quad P_{\perp}=\left(P_{1}, P_{2}\right)
$$

and

$$
M_{\mu \nu}=\left(\begin{array}{cccc}
0 & -S_{1} & -S_{2} & \frac{1}{2} K_{3} \\
S_{1} & 0 & J_{3} & B_{1} \\
S_{2} & -J_{3} & 0 & B_{2} \\
-\frac{1}{2} K_{3} & -B_{1} & -B_{2} & 0
\end{array}\right)
$$

with the new generators defined as

$$
\begin{aligned}
S_{1} & =\frac{1}{2}\left(K_{1}-J_{2}\right), \\
S_{2} & =\frac{1}{2}\left(K_{2}+J_{1}\right), \\
B_{1} & =\frac{1}{2}\left(K_{1}+J_{2}\right), \\
B_{2} & =\frac{1}{2}\left(K_{2}-J_{1}\right) .
\end{aligned}
$$

The relations among the operators $P^{-}, P^{1}, P^{2}, P^{+}, J_{3}, B_{1}, B_{2}$ are the same as the relations between the symmetry operators of the non-relativistic Galilean dynamics in two dimensions [4, 35]. We thus have

$$
\begin{aligned}
{\left[P^{+}, P_{\perp}\right] } & =\left[P^{-}, P_{\perp}\right]=\left[P^{+}, P^{-}\right]=\left[J_{3}, P^{-}\right]=\left[J_{3}, P^{+}\right]=\left[B_{1,2}, P^{+}\right]=0, \\
{\left[J_{3}, P^{k}\right] } & =i \epsilon_{k l} P^{l} \\
{\left[J_{3}, B^{k}\right] } & =i \epsilon_{k l} B^{l} \\
{\left[B_{k}, P^{-}\right] } & =-i P^{k} \\
{\left[B_{k}, P^{l}\right] } & =-i \delta_{k l} P^{+}
\end{aligned}
$$

with $\epsilon_{12}=-\epsilon_{21}=1$ and $\epsilon_{11}=\epsilon_{22}=0$. We can thus identify the different operators with the Galilean dynamics if we make the correspondence $P^{-} \rightarrow H, P^{+} \rightarrow$ mass, $\vec{P}_{\perp} \rightarrow 2$-dim. momentum, $J_{3} \rightarrow$ angular momentum and $B_{1}$ and $B_{2}$ the generators of the Galilean boosts in transverse directions 1 and 2 respectively. Thus there is an isomorphism between the subgroup of the Poincaré group in the front-form and the Galilean symmetry group in two dimensions [3, 4, 35]. 
An example of this analogy can be illustrated in terms of the mass shell condition for the single particle

$$
m^{2}=p^{\mu} p_{\mu}=p^{+} p^{-}-\vec{p}_{\perp}^{2},
$$

which, upon solving for $p^{-}$, gives the Hamiltonian for the free particle in the form

$$
p^{-}=H=\frac{\vec{p}_{\perp}^{2}}{p^{+}}+V_{0},
$$

where $V_{0}=\frac{m^{2}}{p^{+}}$is the constant potential. In the Lorentz group one can identify a kinematical subgroup for each of the reference frames. This subgroup leaves the space-like hyper surface of the constant time invariant. In the instant time formulation the kinematical subgroup is formed by the translations and rotations and thus there are 6 kinematical generators. The remaining generators are referred to as the dynamical generators.

In the case of the light-front formulation, the kinematical generators are

$$
P^{1}, P^{2}, P^{+}, J_{3}, K_{3}, B_{1}, B_{2},
$$

and note that there are 7 of these in contrast with 6 for the instant time $[1,2,4,35]$. The remaining ones are $S_{1}, S_{2}, P^{-}$which are the dynamical ones. Finally, we shall mention that the operator $K_{3}$ has the property that it rescales the other operators in the following manner

$$
\begin{aligned}
\exp \left(i \omega K_{3}\right) \eta \exp \left(-i \omega K_{3}\right) & =e^{\omega} P^{+} \\
\exp \left(i \omega K_{3}\right) \vec{P}_{\perp} \exp \left(-i \omega K_{3}\right) & =\vec{P}_{\perp} \\
\exp \left(i \omega K_{3}\right) P^{-} \exp \left(-i \omega K_{3}\right) & =e^{-\omega} P^{-} \\
\exp \left(i \omega K_{3}\right) J_{3} \exp \left(-i \omega K_{3}\right) & =J_{3} \\
\exp \left(i \omega K_{3}\right) \vec{B} \exp \left(-i \omega K_{3}\right) & =e^{\omega} \vec{B} \\
\exp \left(i \omega K_{3}\right) \vec{S} \exp \left(-i \omega K_{3}\right) & =e^{-\omega} \vec{S}
\end{aligned}
$$

\subsection{Old-fashioned time ordered perturbation theory}

In order to formulate quantum theory on the light-front one can use two approaches. One can directly perform the coordinate change and find the Hamiltonian on the light-front, together with the corresponding equations of motion. The commutation relations for the fields will be formulated for the equal light-front time [4, 35-38]. One can then find the corresponding rules for the perturbation theory. The other approach is to start from the old-fashioned perturbation theory and develop the rules from the form of the expansion in the infinite momentum frame $[4,9,35,37]$. In the following we shall use the second approach for the scalar theory and later on consider the gauge theory.

Following [4] we shall consider first a scalar theory which has the interaction of $g \int d^{3} x \phi^{3}(x)$, where $g$ is the coupling. The Hamiltonian is equal to $H=H_{0}+V$ where the free part is defined as

$$
H_{0}=\int \frac{d^{3} \vec{k}}{E_{k}} \phi^{+}(k) \phi^{-}(k) E_{k}
$$

and the operators $\phi^{+}$and $\phi^{-}$are related to the usual creation and annihilation operators via

$$
\phi^{+}(k)=\sqrt{2 E_{k}} a^{\dagger}(k), \quad \phi^{-}(k)=\sqrt{2 E_{k}} a^{-}(k),
$$

where the energy has been defined as

$$
E_{k}=\sqrt{\vec{k}^{2}+m^{2}}
$$


Here, $\vec{k}$ is the 3 -momentum and $m$ the mass of the scalar particle. The standard commutation relations are defined as

$$
\left[\phi^{-}(k), \phi^{+}\left(k^{\prime}\right)\right]=2 E_{k} \delta^{3}\left(\vec{k}-\vec{k}^{\prime}\right) .
$$

Using these operators one can express the interaction in the following form

$$
V=g \int \frac{d^{3} \vec{k}}{2 E_{k}} \int \frac{d^{3} \vec{l}}{2 E_{l}} \int \frac{d^{3} \vec{q}}{2 E_{q}}\left[\phi^{+}(k) \phi^{+}(l) \phi^{+}(q)+3 \phi^{+}(k) \phi^{+}(l) \phi^{-}(q)\right] \delta^{3}(\vec{k}+\vec{l}+\vec{q})+\text { h.c. }
$$

One can then develop perturbation theory by computing the transition amplitude for the process $i \rightarrow f$

$$
\langle f|T| i\rangle,
$$

where the scattering matrix operator $T$ has the following perturbative expansion in the LippmanSchwinger formalism

$$
T=V+V \frac{1}{E_{i}-H_{0}+i \epsilon} V+V \frac{1}{E_{i}-H_{0}+i \epsilon} V \frac{1}{E_{i}-H_{0}+i \epsilon} V+\ldots
$$

The $E_{i}$ is the energy of the initial state. It is understood that the complete set of Fock states are inserted between the vertices $V$. The operators $\frac{1}{E_{i}-H_{0}+i \epsilon}$ which act on the intermediate states will give energy denominators

$$
\frac{1}{E_{i}-\sum_{\mathrm{int}} E_{\mathrm{int}}}
$$

where $\sum_{\text {int }} E_{\text {int }}$ is the sum of energies in the corresponding intermediate state. Inspecting the structure of the interaction given in Eq. 32 we see that there could be four different types of transitions, all of which are illustrated in Fig. 1.
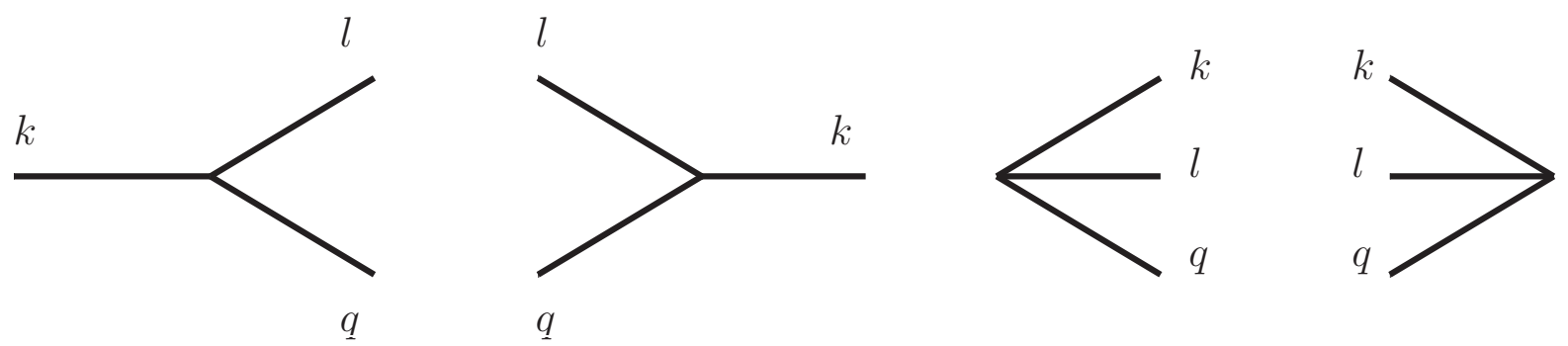

Figure 1: Four types of transitions for the scalar $\phi^{3}$ theory.

The perturbation rules by which one evaluates the individual contributions to the transition amplitude are the following

1. Draw all possible diagrams with given number of vertices, say $n$, for the process under consideration.

2. For each topologically distinct diagram, draw all the possible time-ordered diagrams, with different time-orderings for the vertices. There will be $n$ ! such time-ordered graphs.

3. For each vertex include the 3-momentum conserving delta function.

4. Each intermediate state will contribute a factor $\frac{1}{E_{i}-\sum_{\mathrm{int}} E_{\mathrm{int}}}$.

5. For each internal line include the phase space factor $\frac{d^{3} \vec{k}}{2 E_{k}}$ 
6. Integrate over each internal momenta in the loops.

7. Sum over time orderings.

8. Sum over all the topologically distinct diagrams.

In the old-fashioned time-ordered perturbation theory the individual diagrams are not Lorentz invariant, however the sum over all the time orderings is Lorentz invariant. This can be easily shown on the following example shown in Fig. 2 for which the contributions from both time-ordered diagrams can be written as

$$
\left\langle p_{1}^{\prime}, p_{2}^{\prime}\left|T_{A}\right| p_{1}, p_{2}\right\rangle=\frac{g^{2}}{2 E\left(p_{1}+p_{2}\right)\left(E\left(p_{1}\right)+E\left(p_{2}\right)-E\left(p_{1}+p_{2}\right)\right)}
$$

and

$$
\left\langle p_{1}^{\prime}, p_{2}^{\prime}\left|T_{B}\right| p_{1}, p_{2}\right\rangle=\frac{g^{2}}{2 E\left(p_{1}+p_{2}\right)\left(-E\left(p_{1}\right)-E\left(p_{2}\right)-E\left(p_{1}+p_{2}\right)\right)}
$$

The sum of these diagrams gives

$$
\begin{gathered}
\left\langle p_{1}^{\prime}, p_{2}^{\prime}\left|T_{A}+T_{B}\right| p_{1}, p_{2}\right\rangle=\frac{g^{2}}{-2 E\left(p_{1}+p_{2}\right)} \frac{2 E\left(p_{1}+p_{2}\right)}{E^{2}\left(p_{1}+p_{2}\right)-\left(E\left(p_{1}\right)+E\left(p_{2}\right)\right)^{2}}= \\
g^{2} \frac{1}{\left(E\left(p_{1}\right)+E\left(p_{2}\right)\right)^{2}-\left(p_{1}+p_{2}\right)^{2}-m^{2}}=\frac{g^{2}}{s-m^{2}},
\end{gathered}
$$

which is manifestly Lorentz invariant.
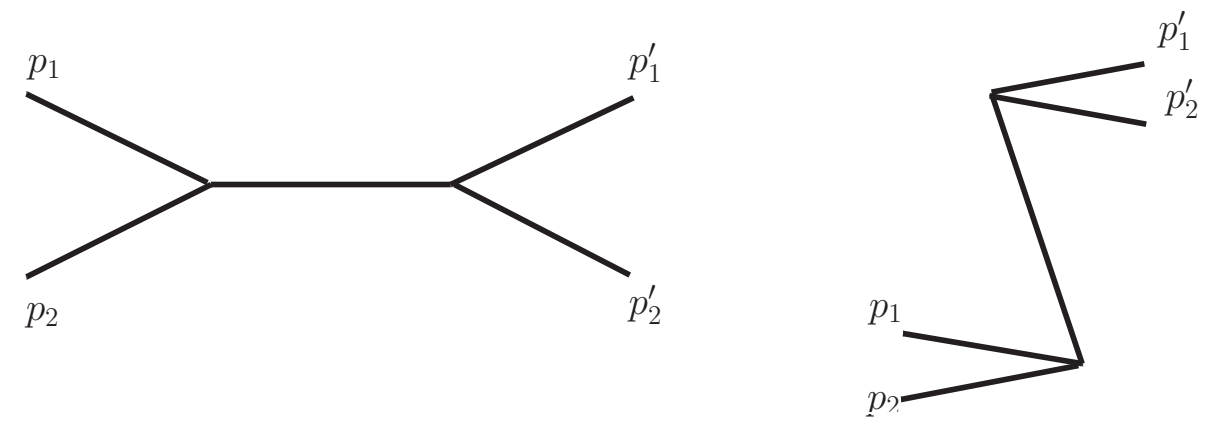

Figure 2: Lowest order time-ordered diagrams for $2 \rightarrow 2$ in the scalar $\phi^{3}$ theory. The time flows from the left to the right side of each diagram. The diagram on the right-hand side contains intermediate state in which the particle moves backward in time. In the infinite momentum frame the contribution from this diagram vanishes and only the diagram on the left hand-side contributes to the total amplitude.

Now, since the individual diagrams are not Lorentz invariant, one can ask what happens to them when different frame is chosen [9]. Let us therefore consider a situation when the reference frame is chosen such that the total momentum $\vec{P}$ is very large. In particular, we shall specify that the component along the $z$ axis is very large. This corresponds to the observer moving with the $\vec{P}$ momentum in the, opposite, $-z$ direction. In that case, the $\vec{p}_{k}$ which is the three momentum of the particle $k$ can be written as

$$
\vec{p}_{k}=z_{k} \vec{P}+\vec{p}_{k \perp},
$$

Here, $\vec{p}_{k \perp}$ is the 2-dimensional transverse momentum such that

$$
\vec{P} \cdot \vec{p}_{k \perp}=0 .
$$


For example, we can set $\vec{P}=\left(0,0, P_{z}\right)$ and $\vec{p}_{k \perp}=\left(p_{k}^{(1)}, p_{k}^{(2)}, 0\right)$. Since $P_{z}$ is kept large, and it is total momentum, it will be convenient in the following to use $z_{k}$ which are fractions of the longitudinal momentum carried by the particles. Because $P_{z}$ is the total momentum we shall have

$$
\sum_{k} \vec{p}_{k \perp}=0, \quad \sum_{k} z_{k}=1
$$

where the sum goes over all the particles in the initial or final state. Now we shall perform the limit of the infinite momentum frame, which means we take the limit of $P_{z} \rightarrow \infty$. In that case, from the point of the view of the observer, the particles are all moving with the large $z$ components of their momenta, such that $z_{k}>0$. The energy of the intermediate state can be expressed as

$$
E_{\text {int }}=\sum_{k} \sqrt{z_{k}^{2} P_{z}^{2}+\vec{p}_{k \perp}^{2}+m_{k}^{2}}=\sum_{k}\left(\left|z_{k}\right| P_{z}+\frac{\vec{p}_{k \perp}^{2}+m_{k}^{2}}{2\left|z_{k}\right| P_{z}}\right)+\mathcal{O}\left(P_{z}^{-2}\right) .
$$

If all the intermediate values of longitudinal momenta are positive, $z_{k}>0$, then we can rewrite the energy of the intermediate state as

$$
E_{\mathrm{int}}=P_{z}+\sum_{k} \frac{\vec{p}_{k \perp}^{2}+m_{k}^{2}}{2 z_{k} P_{z}}+\mathcal{O}\left(P_{z}^{-2}\right)
$$

where we have used the statement of the conservation of the longitudinal momentum fraction $\sum_{k} z_{k}=1$. This is also true for the initial and final state. Therefore if one takes the energy denominator which is the difference between the initial and intermediate states energy we obtain

$$
E_{\text {initial }}-E_{\text {int }}=\sum_{k, \text { initial }} \frac{\vec{p}_{k \perp}^{2}+m_{k}^{2}}{2 z_{k} P_{z}}-\sum_{k, \text { int }} \frac{\vec{p}_{k \perp}^{2}+m_{k}^{2}}{2 z_{k} P_{z}}
$$

we see that the large momentum $P_{z}$ canceled out, and we have kept the leading terms in the large $P_{z}$ limit (the subleading terms will not contribute to the amplitudes if we take the infinite momentum limit $\left.P_{z} \rightarrow \infty\right)$. In that case the diagram which contains such denominator will get a factor

$$
\frac{1}{E_{\text {initial }}-E_{\text {int }}}=\frac{2 P_{z}}{\sum_{k, \text { initial }} \frac{\vec{p}_{k \perp}^{2}+m_{k}^{2}}{z_{k}}-\sum_{k, \text { int }} \frac{\vec{p}_{k \perp}^{2}+m_{k}^{2}}{z_{k}}} .
$$

Consider now the situation, when some intermediate state has $z_{k}<0$, i.e. backward going line. In this case we shall have

$$
\sum_{k, \text { initial }} z_{k}-\sum_{k, \text { int }}\left|z_{k}\right| \neq 0
$$

and consequently there will be a large term in the energy differences, i.e.

$$
E_{\text {initial }}-E_{\text {int }}=P_{z}\left(1-\sum_{k, \text { int }}\left|z_{k}\right|\right)+\sum_{k, \text { initial }} \frac{\vec{p}_{k \perp}^{2}+m_{k}^{2}}{2 z_{k} P_{z}}-\sum_{k, \text { int }} \frac{\vec{p}_{k \perp}^{2}+m_{k}^{2}}{2\left|z_{k}\right| P_{z}}
$$

Therefore the leading behavior of the energy denominators in the $P_{z} \rightarrow \infty$ limit is

$$
\frac{1}{E_{\text {initial }}-E_{\text {int }}}=\frac{1}{P_{z}\left(1-\sum_{k, \text { int }}\left|z_{k}\right|\right)} .
$$

We see that the (leading) contribution of this diagram is suppressed by a power of $P_{z}^{2}$ with respect to the previous diagram. Combining all the phase space factors together with the energy denominators, it 
can be shown that the diagrams where all the internal lines have $z_{k}>0$ are finite, whereas the diagrams in which $l$ intermediate lines are having $z_{k}<0$ are suppressed by power of $1 / P_{z}^{2 l}$ and vanish in the infinite momentum limit of $P_{z} \rightarrow \infty$. Therefore in the infinite momentum frame the vacuum diagrams of the type shown on the right-hand side in Fig. 2, where particles are created or destroyed out of vacuum, vanish and they do not need to be taken into account. Thus this structure of the perturbation theory when viewed in the infinite momentum frame is similar to the old-fashioned perturbation theory applied to the non-relativistic problems. This non-relativistic structure of the perturbation theory is ultimately related to the Galilean subgroup of the Poincaré group on the light-front.

\subsection{Light-front rules for the scalar theory}

We have thus seen in the previous section that the limit of the Lorentz frames in which the $P_{z}$ is taken to infinity results in great simplifications as large class of the vacuum diagrams in time-ordered perturbation theory give vanishing contributions. Thus based on the analysis of the preceding section one can easily write down the new rules of the perturbation theory. We shall do it here for the case of the scalar theory. Gauge theory is discussed in the next section.

1. Write down all possible light-front time ordered diagrams for a given order of perturbation theory. Do not draw the diagrams in which the momenta are flowing backwards in light-front time.

2. Each line will have 2-dimensional transverse momentum $\vec{k}_{\perp}$ and the longitudinal momentum fraction $z_{k}>0$.

3. For each vertex include the conservation of the longitudinal and transverse components

$$
g \delta\left(z_{1}-z_{2}-z_{3}\right) \delta^{(2)}\left(\vec{k}_{1 \perp}-\vec{k}_{2 \perp}-\vec{k}_{3 \perp}\right)
$$

4. For each internal line include the phase space factor

$$
d^{2} \vec{k}_{\perp} \frac{d z}{z} \theta(z)
$$

5. For every intermediate state include the following energy denominator

$$
\frac{1}{E_{\text {initial }}^{-}-E_{\text {int }}^{-}}
$$

where

$$
E^{-} \equiv \sum_{j} \frac{\vec{k}_{j \perp}^{2}+m_{j}^{2}}{z_{j}}
$$

where the sum goes over the different particles in the given state.

6. Integrate over all the internal momenta.

7. Sum over all the time orderings for a given topology of diagram and finally sum over the diagrams.

For the on-shell initial and final states the energies $E^{-}$are conserved (in addition to the conservation of the transverse and longitudinal momenta). As an example we evaluate the contribution from graph 
on the left side of Fig. 2, since under new rules this is the only one which contributes. The rules will give

$$
\begin{gathered}
\left\langle p_{1}^{\prime}, p_{2}^{\prime}\left|T_{A}\right| p_{1}, p_{2}\right\rangle_{L F}=g^{2} \frac{1}{E^{-}\left(p_{1}\right)+E^{-}\left(p_{2}\right)-E^{-}\left(p_{1}+p_{2}\right)}= \\
\frac{g^{2}}{\left(z_{1}+z_{2}\right)\left(E^{-}\left(p_{1}\right)+E^{-}\left(p_{2}\right)\right)-\left(p_{1 \perp}+p_{2 \perp}\right)^{2}-m^{2}}=\frac{g^{2}}{s-m^{2}}
\end{gathered}
$$

which is the same as before. We see that the whole contribution to the scattering comes now from one diagram alone, when one performs the computation in the infinite momentum frame.

\subsection{Light-front formalism for the gauge theory}

\subsubsection{Gluon propagator in the light-front theory}

In the case of the gauge theory, additional complications arise due to the choice of the gauge and the polarization. It turns out that in additional to the standard propagators which are taken care of by the energy denominators one needs to include the instantaneous terms. These are the terms which are like Coulomb terms for the Coulomb gauge choice in the quantum field theory. To find out the decomposition for the gluon propagator let us consider the following expression

$$
D^{\mu \nu}(x)=\frac{1}{(2 \pi)^{4}} \int d^{4} k \exp \left(-i k_{\alpha} x^{\alpha}\right) \frac{-g^{\mu \nu}}{k_{\lambda} k^{\lambda}+i \epsilon},
$$

We shall try to rewrite first this propagator in the form which will allow to derive time-ordered diagrams

$$
D^{\mu \nu}=D_{+}^{\mu \nu} \theta\left(x^{+}\right)+D_{-}^{\mu \nu} \theta\left(-x^{+}\right),
$$

where $x^{+}$is the light-front time. We work in the light-cone gauge, $A^{+}=0$ which is defined by $\eta \cdot A=0$, with vector $\eta$ which is light like and equal to

$$
\eta=(0,0,0,2) .
$$

This choice of the gauge defines the polarization four-vectors of the gluon with four-momentum $k$

$$
\varepsilon_{i}^{ \pm \mu}(\eta) \equiv \varepsilon_{i}^{ \pm \mu}=\varepsilon_{\perp}^{ \pm \mu}+\frac{\vec{\varepsilon}_{\perp}^{ \pm} \cdot \vec{k}_{i \perp}}{k_{i} \cdot \eta} \eta^{\mu},
$$

with

$$
\varepsilon_{\perp}^{ \pm \mu}=\frac{1}{\sqrt{2}}(0,1, \pm i, 0)
$$

It is easy to check that the polarization vectors satisfy the following properties

$$
\varepsilon_{i}^{ \pm} \cdot\left(\varepsilon_{i}^{ \pm}\right)^{*}=-1, \quad \varepsilon_{i}^{ \pm} \cdot\left(\varepsilon_{i}^{\mp}\right)=-1, \quad \varepsilon_{i}^{ \pm} \cdot\left(\varepsilon_{i}^{ \pm}\right)=\varepsilon_{i}^{ \pm} \cdot\left(\varepsilon_{j}^{ \pm}\right)=0, \quad\left(\varepsilon_{i}^{ \pm}\right)^{*}=\varepsilon_{i}^{\mp} .
$$

With this definitions of the gauge and polarization vectors one can find the following decomposition of the projector

$$
d^{\mu \nu} \equiv \sum_{\lambda= \pm} \varepsilon^{\mu}(k, \lambda)\left(\varepsilon^{\nu}(k, \lambda)\right)^{*}=-g^{\mu \nu}+\frac{\eta^{\mu} k^{\nu}+\eta^{\nu} k^{\mu}}{\eta \cdot k}-\frac{\eta^{\mu} \eta^{\nu}}{(\eta \cdot k)^{2}} k^{2} .
$$

It is straightforward to verify that this projector is doubly transverse, i.e. it is transverse both to the vector $\eta$ as well as to the momentum $k$

$$
d^{\mu \nu} \eta_{\nu}=d^{\mu \nu} k_{\nu}=0
$$


Using the projector we thus find that

$$
D^{\mu \nu}(x)=\frac{1}{(2 \pi)^{4}} \int d^{4} k \exp \left(-i k^{\mu} x_{\mu}\right) \frac{d^{\mu \nu}}{k_{\mu} k^{\mu}+i \epsilon}+\frac{1}{(2 \pi)^{4}} \eta^{\mu} \eta^{\nu} \int d^{4} k \exp \left(-i k^{\mu} x_{\mu}\right) \frac{1}{\eta^{2}} \frac{k^{2}}{k_{\mu} k^{\mu}+i \epsilon},
$$

We have omitted the terms proportional to $\eta^{\mu} k^{\nu}$ since they will not contribute to any physical process due to the current conservation.

Writing out explicitly first integral in terms of $k^{+}, k^{-}$and $k_{\perp}$ we can rewrite the propagator as

$$
D^{\mu \nu}(x)=D_{1}^{\mu \nu}(x)+D_{2}^{\mu \nu}(x)
$$

where the two terms are

$$
\begin{gathered}
D_{1}^{\mu \nu}(x)=\frac{1}{2(2 \pi)^{4}} \int d^{2} k_{\perp} d k^{+} d k^{-} \exp \left(-\frac{i}{2} k^{+} x^{-}\right) \exp \left(-\frac{i}{2} k^{-} x^{+}\right) \exp \left(i k_{\perp} \cdot x_{\perp}\right) \frac{d^{\mu \nu}}{k^{+} k^{-}-k_{\perp}^{2}+i \epsilon}, \\
D_{2}^{\mu \nu}(x)=\frac{1}{(2 \pi)^{4}} \eta^{\mu} \eta^{\nu} \int d^{4} k \exp \left(-i k^{\mu} x_{\mu}\right) \frac{1}{\eta^{2}} \frac{k^{2}}{k_{\mu} k^{\mu}+i \epsilon} .
\end{gathered}
$$

The integrand in the first integral contains a pole in $k^{-}$, and therefore we can evaluate this integral by contour integration. If $x^{+}>$then we can close the contour on the lower half complex plane in $k^{-}$, and for $x^{+}<0$ in the upper half plane. The result then for $D_{1}^{\mu \nu}$ reads

$$
D_{1}^{\mu \nu}(x)=\frac{-2 \pi i}{2(2 \pi)^{4}} \int d^{2} k_{\perp} \int_{0}^{\infty} \frac{d k^{+}}{k^{+}} d^{\mu \nu}\left[\exp (-i k \cdot x) \theta\left(x^{+}\right)+\exp (+i k \cdot x) \theta\left(-x^{+}\right)\right]
$$

where $k^{-}=\frac{k_{\perp}^{2}}{k^{+}}$. For the second term, the integrand is actually finite since

$$
\frac{p^{2}}{p^{2}+i \epsilon} \rightarrow 1, \quad \epsilon \rightarrow 0
$$

Therefore the second integral can be evaluated as

$$
D_{2}^{\mu \nu}(x)=\frac{1}{(2 \pi)^{4}} \eta^{\mu} \eta^{\nu} \int d^{4} k \exp \left(-i k^{\mu} x_{\mu}\right) \frac{1}{\eta^{2}}=\frac{1}{(2 \pi)^{3}} \delta\left(x^{+}\right) \int d^{2} k_{\perp} \int_{-\infty}^{\infty} \frac{d \eta}{\eta^{2}} \exp \left(-i \frac{1}{2} x^{-} k^{+}+i x_{\perp} k_{\perp}\right) .
$$

We see that this part is proportional to the Dirac delta in the light-front time, and therefore this is the instantaneous term of the propagator. This is reminiscent of the Coulomb interaction which does appear when quantizing electrodynamics in the Coulomb gauge.

One can repeat the same analysis for the fermion, and also in this case there will be an instantaneous part of the propagator.

\subsubsection{Light-front quantization for QCD}

In this section we outline the steps of the light-front quantization which will lead to the light-front rules of the perturbation theory, for more details see for example [35, 39, 40]. Let us start with the Lagrangian density for the Quantum Chromodynamics which can be written as (we are using here the conventions of paper [39])

$$
\mathcal{L}=-\frac{1}{2} \operatorname{Tr}\left(F^{\mu \nu} F_{\mu \nu}\right)+\bar{\Psi}(i \not D-m) \Psi
$$

where

$$
F^{\mu \nu}=\partial^{\mu} A^{\nu}-\partial^{\nu} A^{\mu}-i g\left[A^{\mu}, A^{\nu}\right], \quad i D^{\mu}=i \partial^{\mu}-g A^{\mu}
$$



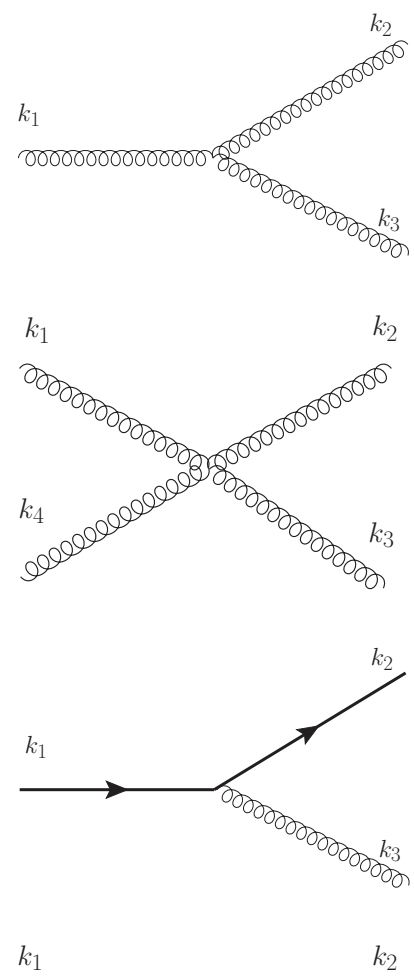

www

$k_{4}$

wwwmow
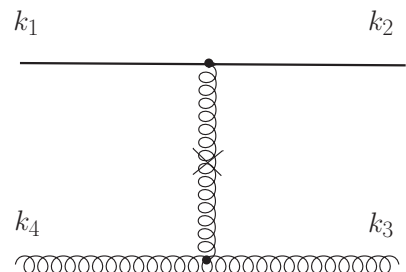

www

$k_{1}$
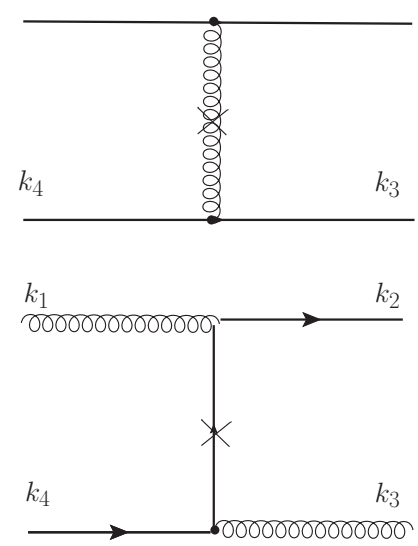

$$
i g\left(g^{\mu_{1} \mu_{2}}\left(k_{1}-k_{2}\right)^{\mu_{3}}+g^{\mu_{2} \mu_{3}}\left(k_{2}-k_{3}\right)^{\mu_{1}}+g^{\mu_{3} \mu_{1}}\left(k_{3}-k_{1}\right)^{\mu_{2}}\right) \varepsilon_{\mu_{1}}^{\lambda_{1}}\left(\varepsilon_{\mu_{2}}^{\lambda_{2}}\right)^{*}\left(\varepsilon_{\mu_{3}}^{\lambda_{3}}\right)^{*}
$$

$$
i g^{2}\left(g^{\mu_{1} \mu_{2}} g^{\mu_{3} \mu_{4}}-g^{\mu_{1} \mu_{4}} g^{\mu_{3} \mu_{2}}\right) \varepsilon_{\mu_{1}}^{\lambda_{1}}\left(\varepsilon_{\mu_{2}}^{\lambda_{2}}\right)^{*}\left(\varepsilon_{\mu_{3}}^{\lambda_{3}}\right)^{*}\left(\varepsilon_{\mu_{4}}^{\lambda_{4}}\right)^{*}+\text { permutations }
$$

$$
g \bar{u}\left(k_{2}\right)\left(\ddagger^{\lambda_{3}}\right)^{*} u\left(k_{1}\right)
$$

$$
i g^{2} \varepsilon^{\lambda_{1}} \cdot \varepsilon^{\lambda_{2} *} \frac{\left(k_{2}^{+}-k_{1}^{+}\right)\left(k_{4}^{+}-k_{3}^{+}\right)}{\left(k_{4}^{+}+k_{1}^{+}\right)^{2}} \varepsilon^{\lambda_{4}} \cdot \varepsilon^{\lambda_{3} *}
$$

$$
g^{2} \bar{u}\left(k_{2}\right) \gamma^{+} u\left(k_{1}\right) \frac{\left(k_{4}^{+}-k_{3}^{+}\right)}{\left(k_{4}^{+}+k_{3}^{+}\right)^{2}} \varepsilon^{\lambda_{3} *} \cdot \varepsilon^{\lambda_{4}}
$$

$$
g^{2} \bar{u}\left(k_{2}\right) \frac{\gamma^{+} u\left(k_{1}\right) \bar{u}\left(k_{3}\right) \gamma^{+}}{\left(k_{4}^{+}-k_{3}^{+}\right)^{2}} u\left(k_{4}\right)
$$

$$
g^{2} \bar{u}\left(k_{2}\right) \ddagger^{\lambda_{1}} \frac{\gamma^{+}}{2\left(k_{4}^{+}-k_{3}^{+}\right)} \oiint^{\lambda_{3} *} u\left(k_{4}\right)
$$

Table 1: Types of interactions in the light-front formulation of QCD. 
Here, $A^{\mu}$ is the gauge field, which is a matrix $A^{\mu}=A^{a \mu} T^{a}$ and $T^{a}$ are the generators of the $S U(3)$ color group. The spinor $\Psi$ is the quark field which is color triplet (we consider here only one flavor, but the whole discussion can be generalized to more flavors). It is useful to introduce the following projection operators

$$
\Lambda_{ \pm}=\frac{1}{2} \gamma^{0} \gamma^{2}, \quad \Lambda_{-} \Lambda_{+}=0, \quad \Lambda_{ \pm}^{2}=\Lambda_{ \pm}, \quad \Lambda_{+}+\Lambda_{-}=1
$$

By using equations of motion $[39,40]$ one can explicitly demonstrate that the only independent dynamical fields are $\Psi_{+}=\Lambda_{+} \Psi$ and $\vec{A}_{\perp}$. The other fields can be expressed in terms of these dynamical fields as follows

$$
\begin{aligned}
\Psi_{-} \equiv \Lambda_{-} \Psi & =\frac{1}{i \partial^{+}}\left[i \vec{D}_{\perp} \cdot \vec{\alpha}_{\perp}+\beta m\right] \Psi_{+} \\
& =\tilde{\Psi}_{-}-\frac{1}{i \partial^{+}} g \vec{A}_{\perp} \cdot \vec{\alpha}_{\perp} \Psi_{+} \\
A^{+} & =0 \\
A^{-} & =\frac{2}{i \partial^{+}} i \vec{\partial}_{\perp} \cdot \vec{A}_{\perp}+\frac{2 g}{\left(i \partial^{+}\right)^{2}}\left(\left[i \partial^{+} A_{\perp}^{i}, A_{\perp}^{i}\right]+2 \Psi_{+}^{\dagger} T^{a} \Psi_{+} T^{a}\right) \\
& =\tilde{A}^{-}+\frac{2 g}{\left(i \partial^{+}\right)^{2}}\left(\left[i \partial^{+} A_{\perp}^{i}, A_{\perp}^{i}\right]+2 \Psi_{+}^{\dagger} T^{a} \Psi_{+} T^{a}\right)
\end{aligned}
$$

where we have defined $\beta=\gamma^{0}$ and $\vec{\alpha}_{\perp}=\gamma^{0} \vec{\gamma}_{\perp}$. One defines the following (anti)commutation relations for the creation and annihilation operators on the light-front

$$
\begin{aligned}
\left\{b\left(k^{+}, \vec{k}_{\perp}, \lambda\right), b^{\dagger}\left(p^{+}, \vec{p}_{\perp}, \lambda^{\prime}\right)\right\} & =16 \pi^{3} k^{+} \delta\left(k^{+}-p^{+}\right) \delta^{2}\left(\vec{k}_{\perp}-\vec{p}_{\perp}\right) \delta_{\lambda \lambda^{\prime}}, \\
\left\{d\left(k^{+}, \vec{k}_{\perp}, \lambda\right), d^{\dagger}\left(p^{+}, \vec{p}_{\perp}, \lambda^{\prime}\right)\right\} & =16 \pi^{3} k^{+} \delta\left(k^{+}-p^{+}\right) \delta^{2}\left(\vec{k}_{\perp}-\vec{p}_{\perp}\right) \delta_{\lambda \lambda^{\prime}}, \\
{\left[a\left(k^{+}, \vec{k}_{\perp}, \lambda\right), a^{\dagger}\left(p^{+}, \vec{p}_{\perp}, \lambda^{\prime}\right)\right] } & =16 \pi^{3} k^{+} \delta\left(k^{+}-p^{+}\right) \delta^{2}\left(\vec{k}_{\perp}-\vec{p}_{\perp}\right) \delta_{\lambda \lambda^{\prime}} .
\end{aligned}
$$

Here, $\lambda$ are the helicities of the quarks and gluons. The spinor and gauge fields at $x^{+}=0$ are defined in terms of these creation and annihilation operators

$$
\begin{aligned}
& \Psi_{+}(x)=\int \frac{d k^{+} d^{2} \vec{k}_{\perp}}{k^{+} 16 \pi^{3}} \theta\left(k^{+}\right) \sum_{\lambda}\left\{b\left(k^{+}, \vec{k}_{\perp}, \lambda\right) u_{+}\left(k^{+}, \vec{k}_{\perp}, \lambda\right) e^{-i k \cdot x}+d^{\dagger}\left(k^{+}, \vec{k}_{\perp}, \lambda\right) v_{+}\left(k^{+}, \vec{k}_{\perp}, \lambda\right) e^{i k \cdot x}\right\}_{x^{+}=0} \\
& A_{\perp}^{i}(x)=\int \frac{d k^{+} d^{2} \vec{k}_{\perp}}{k^{+} 16 \pi^{3}} \theta\left(k^{+}\right) \sum_{\lambda}\left\{a\left(k^{+}, \vec{k}_{\perp}, \lambda\right) \varepsilon_{\perp}^{i}(\lambda) e^{-i k \cdot x}+a^{\dagger}\left(k^{+}, \vec{k}_{\perp}, \lambda\right) \varepsilon_{\perp}^{* i}(\lambda) e^{i k \cdot x}\right\}_{x^{+}=0} .
\end{aligned}
$$

The corresponding canonical commutation relations for the fields at equal light cone time are defined as

$$
\begin{aligned}
\left\{\Psi_{+}\left(x^{-}, \vec{x}_{\perp}\right), \Psi_{+}^{\dagger}\left(x^{-}, \vec{x}_{\perp}\right)\right\} & =\delta\left(x^{-}-y^{-}\right) \delta^{2}\left(\vec{x}_{\perp}-\vec{y}_{\perp}\right) \\
{\left[A_{\perp}^{j}\left(x^{-}, \vec{x}_{\perp}\right), \partial^{+} A_{\perp}^{l}\left(y^{-}, \vec{y}_{\perp}\right)\right] } & =i \delta^{j l} \delta\left(x^{-}-y^{-}\right) \delta^{2}\left(\vec{x}_{\perp}-\vec{y}_{\perp}\right) .
\end{aligned}
$$

These creation and annihilation operators, when acting on the vacuum state $|0\rangle$ define the Fock basis of states, whose evolution is governed by the light-front Hamiltonian $H=P^{-}$. It can be demonstrated, see [39], that the Hamiltonian can be written as a sum of the free part $H_{0}$ and the interaction part $V$

$$
H=H_{0}+V
$$


where the free part is written as

$$
\begin{aligned}
H_{0}= & \sum_{\lambda} \int \frac{d k^{+} d^{2} \vec{k}_{\perp}}{k^{+} 16 \pi^{3}}\left\{a^{\dagger}\left(k^{+}, \vec{k}_{\perp}, \lambda\right) a\left(k^{+}, \vec{k}_{\perp}, \lambda\right) \frac{\vec{k}_{\perp}^{2}}{k^{+}}+b^{\dagger}\left(k^{+}, \vec{k}_{\perp}, \lambda\right) b\left(k^{+}, \vec{k}_{\perp}, \lambda\right) \frac{\vec{k}_{\perp}^{2}+m^{2}}{k^{+}}\right. \\
& \left.d^{\dagger}\left(k^{+}, \vec{k}_{\perp}, \lambda\right) d\left(k^{+}, \vec{k}_{\perp}, \lambda\right) \frac{\vec{k}_{\perp}^{2}+m^{2}}{k^{+}}\right\}
\end{aligned}
$$

and the interaction term can be expressed as

$$
\begin{aligned}
& V=\int d x^{-} d^{2} \vec{x}_{\perp}\{ 2 g \operatorname{Tr}\left(i \partial^{\mu} \tilde{A}^{\nu}\left[\tilde{A}_{\mu}, \tilde{A}_{\nu}\right]\right)-\frac{g^{2}}{2} \operatorname{Tr}\left(\left[\tilde{A}^{\mu}, \tilde{A}^{\nu}\right]\left[\tilde{A}_{\mu}, \tilde{A}_{\nu}\right]\right) \\
&+g \tilde{\tilde{\Psi}} \tilde{A} \tilde{\Psi}+g^{2} \operatorname{Tr}\left(\left[i \partial^{+} \tilde{A}^{\mu}, \tilde{A}_{\mu}\right] \frac{1}{\left(i \partial^{+}\right)^{2}}\left[i \partial^{+} \tilde{A}^{\nu}, \tilde{A}_{\nu}\right]\right) \\
&\left.+g^{2} \bar{\Psi} \tilde{A} \frac{\gamma^{+}}{2 i \partial^{+}} \tilde{A} \tilde{\Psi}-g^{2} \overline{\tilde{\Psi}} \gamma^{+}\left(\frac{1}{\left(i \partial^{+}\right)^{2}}\left[i \partial^{\mu} \tilde{A}^{\mu}, \tilde{A}_{\mu}\right]\right) \tilde{\Psi}+\frac{g^{2}}{2} \bar{\Psi} \gamma^{+} T^{a} \Psi \frac{1}{\left(i \partial^{+}\right)^{2}} \bar{\Psi} \gamma^{+} T^{a} \Psi\right\}
\end{aligned}
$$

where $\tilde{\Psi}=\tilde{\Psi}_{-}+\Psi_{+}$and $\tilde{A}^{\mu}=\left(0, \tilde{A}^{-}, \vec{A}_{\perp}\right)$. The first two terms in the $V$ correspond to the selfinteraction (triple and four-gluon vertices) of the non-abelian theory. The third term corresponds to the standard quark-gluon vertex. The remaining terms correspond to the instantaneous interactions between quarks and gluons. From the Hamiltonian it is possible to derive the rules of the light - front theory with the interactions which are listed in Table 1.

\subsubsection{Light-front rules for the QCD}

We can now summarize the rules for the light-front perturbation theory in the case of QCD:

1. Assign a momentum $k^{\mu}$ to each line. Longitudinal $k^{+}$and transverse $\vec{k}_{\perp}$ momenta components are conserved at each vertex and $k^{2}=m^{2}$, i.e. is on mass-shell. Therefore

$$
k^{-}=\frac{\vec{k}_{\perp}^{2}}{k^{+}} .
$$

2. Assign a factor

$$
\frac{\theta\left(k^{+}\right)}{k^{+}}
$$

to each internal line. Thus all the particles are moving forward, and this eliminates large class of vacuum diagrams. Sometimes a convention is used in which $1 / k^{+}$factor is absorbed in the vertices, in this way vertices get $1 / \sqrt{k^{+}}$factors from all lines.

3. For each vertex assign an appropriate factor, the three-gluon, four-gluon and fermion-gluon vertices are listed in Table 1.

4. For each intermediate state include the energy denominator

$$
\frac{1}{\sum_{i, \text { initial }} E_{i}^{-}-\sum_{i, \text { int }} E_{i}^{-}+i \epsilon},
$$

5. Since the fermion propagator has instantaneous term $\gamma^{+} / 2 k^{+}$as well as the gluon propagator $\eta^{\mu} \eta^{\nu} / k^{+2}$ we have to include them as separate graphs. These instantaneous interactions are listed 
in Table 1. Alternatively one can redefine the energy denominator to include the instantaneous interaction inside. This is done by defining auxiliary momentum [41]

$$
\tilde{k}^{\mu}=\left(k^{+}, \sum_{i, \text { initial }} E_{i}^{-}-\sum_{i, \text { int }^{\prime}} E_{i}^{-}, \vec{k}_{\perp}\right)
$$

where the sum $\sum_{\text {int }^{\prime}}$ means that one sums over all the particles in the given intermediate state except the particle of interest. This new momentum replaces $k$ in the polarization sum and in the triple-gluon vertex. Similarly for the fermions, where the sum $\sum_{s} u_{s}(k) \bar{u}_{s}(k)$ is replaced by $\tilde{k}+m$ and the $\operatorname{sum} \sum_{s} v_{s}(k) \bar{v}_{s}(k)$ by $\tilde{\not k}-m$.

6. Perform the integrals over

$$
\frac{d^{2} \vec{k}_{\perp}}{2(2 \pi)^{3}} d k^{+}
$$

7. Perform the summation over the colors as in the usual covariant diagrams.

\section{Scattering amplitudes in the helicity formalism}

We shall be ultimately interested in the computation of the scattering amplitudes using the light-front methods. It will be of interest however, to review briefly helicity methods for the computation of the scattering amplitudes since there are close connections between the two methods. The amplitudes in the helicity representation take on particularly simple and elegant forms in terms of the spinor products. On the other hand, as we shall see later, the spinor products emerge naturally in the light-front formalism due to the Galilean structure of the light-front dynamics. To be precise, we shall see that the spinor products are proportional to the variables which can be interpreted as the relative light-front 'velocities' of the emitted particles in the scattering amplitude. There are many excellent references and reviews of the helicity methods in gauge theories, see for example [42-45]. There are also many reviews, which discuss the methods for the computations of loop QCD amplitudes, [46-49] and in $N=4$ super-YangMills theory $[50,51]$. Therefore we do not aim to perform here a complete review of the helicity methods but rather we shall recall only basic properties which will be pertinent for the later derivation of the tree-level amplitudes using the light-front perturbation theory. We shall also briefly discuss two important recursion relations which can be used to compute the amplitudes: Berends-Giele recursion relations [20] which use off-shell currents and Britto-Cachazo-Feng-Witten recursion relations [21, 22] which use decomposition of the amplitudes into on-shell subamplitudes with shifted complex momenta. The notation and conventions used here are closely following that used in [44]. For more details we recommend the reader to consult these excellent reviews [43-45].

\subsection{Helicity formalism}

The helicity formalism for the calculation of the multi-parton scattering in massless QED case was first used in $[52,53]$ for the calculation of the trident production of $e^{+} e^{-}$pairs (a production of a charged lepton pair by a charged lepton incident on a nuclear target). It was then later developed in $[54-56]$. The idea is to calculate the matrix elements for massless particles with the final states which have definite assigned helicity. Since the different helicity configurations do not interfere with each other, one can sum incoherently the squares of the amplitudes which contribute to the process in question. Furthermore, one can exploit the property of the gauge invariance, which manifests itself in the freedom of the choice of the reference momentum for the polarization vectors. This can be used to choose different polarization vectors for each gauge invariant component of the amplitude and thus can lead to important simplifications in the evaluation of the diagrams. 
First, let us consider massless fermions. In this case the solutions to the Dirac equation of positive and negative energies are proportional to each other since we have

$$
\not k u(k)=\not k v(k)=0 \text {. }
$$

One can thus introduce the states with definite helicities by using the projection operators

$$
u_{ \pm}(k)=P_{ \pm} u(k), \quad v_{ \pm}(k)=P_{\mp} v(k)
$$

which are defined as

$$
P_{ \pm}=\frac{1}{2}\left(1 \pm \gamma_{5}\right)
$$

Recall that the helicity eigenstates coincide with the eigenspinors of $\gamma_{5}$ and that the helicity for negative solutions is negative of the $\gamma_{5}$ eigenvalue. Therefore it follows that

$$
u_{ \pm}(k)=v_{\mp}(k), \quad \overline{u_{ \pm}(k)}=\overline{v_{\mp}(k)} .
$$

For the scattering amplitudes with many gluons, there are many external momenta, we shall label them $k_{i}$ where $i=1, \ldots, n$, where $n$ is the number of external legs, and use the standard notation

$$
\left|k_{i} \pm\right\rangle=u_{ \pm}\left(k_{i}\right), \quad\left\langle k_{i} \pm\right|=\overline{u_{ \pm}\left(k_{i}\right)} .
$$

From these definitions one can construct the spinor products

$$
\langle i-\mid j+\rangle=\overline{u_{-}\left(k_{i}\right)} u_{+}\left(k_{j}\right), \quad\langle i+\mid j-\rangle=\overline{u_{+}\left(k_{i}\right)} u_{-}\left(k_{j}\right) .
$$

It is standard to use the following notation, by introducing two kinds of 'brackets'

$$
\langle i j\rangle \equiv\langle i-\mid j+\rangle, \quad[i j] \equiv\langle i+\mid j-\rangle .
$$

From the properties of the Dirac algebra one can prove that the spinor products are antisymmetric

$$
\langle i j\rangle=-\langle j i\rangle, \quad[i j]=-[j i],
$$

and from the property of the projector $P_{\mp} P_{ \pm}=0$ one can easily show that the following products vanish identically

$$
\langle i+\mid j+\rangle=[i j\rangle=0=\langle i-\mid j-\rangle=\langle i j] .
$$

In order to find out the explicit expressions for the spinor products one needs to use some representation of the spinors. Following [44] we shall use Dirac representation for the gamma matrices

$$
\gamma^{0}=\left(\begin{array}{cc}
\mathbb{1} & 0 \\
0 & -\mathbb{1}
\end{array}\right), \quad \gamma^{i}=\left(\begin{array}{cc}
0 & \sigma^{i} \\
-\sigma^{i} & 0
\end{array}\right), \quad \gamma^{5}=\left(\begin{array}{ll}
0 & \mathbb{1} \\
\mathbb{1} & 0
\end{array}\right), \quad P_{ \pm}=\frac{1}{2}\left(\begin{array}{cc}
\mathbb{1} & \pm \mathbb{1} \\
\pm \mathbb{1} & \mathbb{1}
\end{array}\right)
$$

In this representation the explicit forms for the massless spinors are

$$
u_{+}(k)=v_{-}(k)=\frac{1}{\sqrt{2}}\left(\begin{array}{c}
\sqrt{k^{+}} \\
\sqrt{k^{-}} e^{i \phi_{k}} \\
\sqrt{k^{+}} \\
\sqrt{k^{-}} e^{i \phi_{k}}
\end{array}\right), \quad u_{-}(k)=v_{+}(k)=\frac{1}{\sqrt{2}}\left(\begin{array}{c}
\sqrt{k^{-}} e^{-i \phi_{k}} \\
-\sqrt{k^{+}} \\
-\sqrt{k^{-}} e^{-i \phi_{k}} \\
\sqrt{k^{+}}
\end{array}\right),
$$

where the phase factor is defined as

$$
e^{ \pm i \phi_{k}}=\frac{k^{(1)} \pm i k^{(2)}}{\sqrt{k^{+} k^{-}}}
$$


Note the appearance of the light-cone variables $k^{+}, k^{-}$in these expressions. Using the above representation of the spinors one can find explicit expressions for the spinor products

$$
\langle i j\rangle=\sqrt{k_{i}^{-} k_{j}^{+}} e^{i \phi_{k_{i}}}-\sqrt{k_{i}^{+} k_{j}^{-}} e^{i \phi_{k_{j}}}=\sqrt{\left|s_{i j}\right|} e^{i \phi_{i j}},
$$

and

$$
[i j]=-\sqrt{k_{i}^{-} k_{j}^{+}} e^{-i \phi_{k_{i}}}+\sqrt{k_{i}^{+} k_{j}^{-}} e^{-i \phi_{k_{j}}}=\sqrt{\left|s_{i j}\right|} e^{-i \phi_{i j}-i \pi},
$$

where we have defined Lorentz invariant sub-energy $s_{i j}=\left(k_{i}+k_{j}\right)^{2}=2 k_{i} \cdot k_{j}$ and the angle $\phi_{i j}$ is defined through

$$
\cos \phi_{i j}=\frac{k_{i}^{(1)} k_{j}^{+}-k_{j}^{(1)} k_{i}^{+}}{\sqrt{\left|s_{i j}\right| k_{i}^{+} k_{j}^{+}}}, \quad \sin \phi_{i j}=\frac{k_{i}^{(2)} k_{j}^{+}-k_{j}^{(2)} k_{i}^{+}}{\sqrt{\left|s_{i j}\right| k_{i}^{+} k_{j}^{+}}} .
$$

Thus we see that the spinor products are complex square roots of the Lorentz product $s_{i j}$

$$
\langle i j\rangle[j i]=s_{i j} .
$$

To be precise, these expressions are valid for the case when both 4-momenta are having positive energy. If one or more of the momenta in $\langle i j\rangle$ have negative energy $\phi_{i j}$ is calculated with the minus momenta which have negative energy and in addition a phase $l \pi / 2$ is added to $\phi_{i j}$ where $l$ is the number of the negative momenta in the spinor product.

The spinor products have also number of properties, which are useful, in particular in the evaluation of the scattering amplitudes in this formalism:

- Gordon identity and the projection operators:

$$
\left\langle i \pm\left|\gamma^{\mu}\right| i \pm\right\rangle=2 k_{i}^{\mu}, \quad|i \pm\rangle\langle i \pm|=P_{ \pm} \not k_{i}
$$

- Fierz rearrangement

$$
\left\langle i+\left|\gamma^{\mu}\right| j+\right\rangle\left\langle k+\left|\gamma_{\mu}\right| l+\right\rangle=2[i k]\langle l j\rangle
$$

- Charge conjugation of the current

$$
\left\langle i+\left|\gamma^{\mu}\right| j+\right\rangle=\left\langle j-\left|\gamma^{\mu}\right| i-\right\rangle
$$

- Shouten identity:

$$
|i\rangle\langle j k\rangle+|j\rangle\langle k i\rangle+|k\rangle\langle i j\rangle=0
$$

In addition, in the $n$-point amplitude, due to the momentum conservation $\sum_{i=1}^{n} k_{i}=0$, there is one more identity

$$
\sum_{\substack{i=1 \\ i \neq j, k}}^{n}[j i]\langle i k\rangle=0
$$

\subsubsection{Polarization vectors}

In order to be able to construct the amplitudes with external particles being the vector bosons we need to construct the polarization vectors. It turns out that we can use spinors since there is a transformation which relates the polarization vectors and spinors. In other words one can construct a Lorentz vector from the combination of spinors and $\gamma^{\mu}$ matrices. More precisely one can write in general

$$
\varepsilon_{\mu}^{+}(k, q)=A\left\langle q-\left|\gamma_{\mu}\right| k-\right\rangle, \quad \varepsilon_{\mu}^{-}(k, q)=A^{*}\left\langle q+\left|\gamma_{\mu}\right| k+\right\rangle,
$$


where $A$ is a complex normalization (to be determined) and where we have used the condition that the complex conjugation reverse the helicity

$$
\varepsilon_{\mu}^{-}(k, q)=\left(\varepsilon_{\mu}^{+}(k, q)\right)^{*}
$$

which resulted in the complex conjugate $A^{*}$ in the second term in Eq. (94). Here, vector $q$ is the reference momentum for the polarization vector. It is reflecting the freedom of the gauge transformations in the on-shell amplitude. Note that, since $\not k|k \pm\rangle=0$ always, therefore $\varepsilon_{\mu}$ is constructed in such a way that it is transverse to $k$ for any value of $q$

$$
\varepsilon^{ \pm}(k, q) \cdot k=0 \text {. }
$$

One can use the normalization condition for the polarization vectors to fix the constant $A$,

$$
\varepsilon^{+} \cdot\left(\varepsilon^{+}\right)^{*}=\varepsilon^{+} \cdot \varepsilon^{-}=-1=-2|A|^{2} q \cdot k,
$$

where we have used the Fierz rearrangement and the charge conjugation property. Therefore one obtains finally the following representation for the polarization vectors

$$
\begin{aligned}
& \varepsilon_{\mu}^{+}(k, q)=e^{i \theta(k, q)} \frac{\left\langle q-\left|\gamma_{\mu}\right| k-\right\rangle}{\sqrt{2}\langle q k\rangle}, \\
& \varepsilon_{\mu}^{-}(k, q)=e^{-i \theta(k, q)} \frac{\left\langle q+\left|\gamma_{\mu}\right| k+\right\rangle}{\sqrt{2}[k q]},
\end{aligned}
$$

which still contains additional phase $\theta(k, q)$ which can depend on both the momentum of the particle $k$ and the reference momentum $q$. In the standard conventions [43-45], this phase is set to zero. These are polarization vectors $\varepsilon^{ \pm}$which describe the outgoing vector bosons with \pm helicity.

It is interesting to see what happens when the reference momentum is changed from $q$ to $q^{\prime}$. Using the above definitions we have that

$$
\begin{aligned}
\varepsilon_{\mu}^{+}\left(k, q^{\prime}\right)-\varepsilon_{\mu}^{+}(k, q)=\frac{\left\langle q^{\prime}-\left|\gamma_{\mu}\right| k-\right\rangle}{\sqrt{2}\left\langle q^{\prime} k\right\rangle}-\frac{\left\langle q-\left|\gamma_{\mu}\right| k-\right\rangle}{\sqrt{2}\langle q k\rangle} & =\frac{\left\langle q^{\prime}-\left|\gamma_{\mu}\right| k-\right\rangle\langle q k\rangle-\left\langle q-\left|\gamma_{\mu}\right| k-\right\rangle\left\langle q^{\prime} k\right\rangle}{\sqrt{2}\left\langle q^{\prime} k\right\rangle\langle q k\rangle}= \\
& =-\frac{\sqrt{2}\left\langle q^{\prime} q\right\rangle}{\left\langle q^{\prime} k\right\rangle\langle q k\rangle} k^{\mu} .
\end{aligned}
$$

For on-shell gauge invariant amplitude the term proportional to $k^{\mu}$ vanishes, when contracted with the amplitude, due to the Ward identity. The freedom of choosing the auxiliary reference momentum can be exploited to make the computations of the amplitudes easier. For example, one can choose different reference momenta for different gauge invariant parts of the full amplitude. There are few handy identities which can be used to make simplifications in the calculations. In particular the following identities are useful:

$$
\begin{aligned}
\varepsilon_{i}^{ \pm}(q) \cdot q & =0, \\
\varepsilon_{i}^{+} \cdot \varepsilon_{j}^{+}(q) & =\varepsilon_{i}^{-} \cdot \varepsilon_{j}^{-}(q)=0, \\
\varepsilon_{i}^{+}\left(k_{j}\right) \cdot \varepsilon_{j}^{-}(q) & =\varepsilon_{i}^{+}(q) \cdot \varepsilon_{j}^{-}\left(k_{i}\right)=0, \\
\oiint_{i}^{+}\left(k_{j}\right)|j\rangle & \left.=\oiint_{i}^{-}\left(k_{j}\right) \mid j\right]=0, \\
{\left[j \mid \ddagger_{i}^{-}\left(k_{j}\right)\right.} & =\langle j| \ddagger_{i}^{+}\left(k_{j}\right)=0 .
\end{aligned}
$$

In the above we have introduced short-cut notation $\varepsilon\left(k_{i}, q\right) \equiv \varepsilon_{i}(q)$. These identities suggest that it is useful to choose the reference momenta of like-helicity gluons to be the same and to be equal to the external momentum of one of the gluons from the opposite helicity set. Finally, we shall note an 
important property of the polarization vectors. One can prove that the completeness relation for these polarization vectors is that of the axial gauge

$$
\sum_{\lambda= \pm} \varepsilon_{\mu}^{\lambda}(k, q)\left(\varepsilon_{\nu}^{\lambda}(k, q)\right)^{*}=-\eta_{\mu \nu}+\frac{k_{\mu} q_{\nu}+k_{\nu} q_{\mu}}{k \cdot q} .
$$

This should be compared with Eq. (56) which is valid in the light-cone gauge. The expressions are very similar if one can identify the reference momentum $q$ with the light-like vector $\eta$ of the light-front perturbation theory. This property will result in the fact that the calculations in the light-front which are performed in the light-cone gauge are simplified. Light-cone gauge (but not light-front perturbation theory) was for example used in the work [57] to compute helicity amplitudes from the recurrence relations.

\subsubsection{Color ordering}

So far we have discussed the degrees of freedom related to the kinematics, in QCD however one needs to take into account the complicated color structure. In general computation of any amplitude in QCD, even at the tree level, is very challenging due to the rapidly increasing number of terms with the increase of the number of external legs. In addition one needs to include the color structure, which can be very complicated even for the simple graphs. Fortunately, there exists simplification which exploits the gauge invariance. The idea is to divide the process into appropriate gauge invariant components. It turns out that the gauge invariant subsets are invariant under cyclic permutations of the external gluons.

This decomposition was proposed in works of [58-61] for the tree level amplitudes. A thorough analysis of the relation between color structures and gauge invariance was done in [62]. The color decomposition principle was extended beyond the tree level to loop amplitudes in [63].

Let us analyze the color factor for any Feynman diagram in QCD. The gauge group for QCD is $S U\left(N_{c}\right)$ with $N_{c}=3$. The gluons carry adjoint index $a=1, \ldots, N_{c}^{2}-1$ and quarks(antiquarks) index $i(\bar{i})=1, \ldots, N_{c}$. The generators of the $S U\left(N_{c}\right)$ group in the fundamental representation are hermitian, traceless $N_{c} \times N_{c}$ matrices which we denote by $\left(T^{a}\right)_{i}^{\bar{j}}$. They satisfy the following standard relations

$$
\left[T^{a}, T^{b}\right]=i f^{a b c} T^{c}, \quad \operatorname{Tr}\left(T^{a} T^{b}\right)=\delta^{a b},
$$

with $f^{a b c}$ being the group structure constant. If we consider any tree level gluon diagram, then each three gluon vertex will contain factor $f^{a b c}$ and the four gluon vertex will contain the color factor $f^{a b e} f^{c d e}$.

One can simplify the color structure by replacing the structure constants $f^{a b c}$ by the matrices $T$ using the following identity

$$
f^{a b c}=-i \operatorname{Tr}\left(T^{a} T^{b} T^{c}-T^{c} T^{b} T^{a}\right),
$$

as well as

$$
\left(T^{a}\right)_{i_{1}}^{\bar{j}_{1}}\left(T^{a}\right)_{i_{2}}^{\bar{j}_{2}}=\delta_{i_{1}}^{\bar{j}_{2}} \delta_{i_{2}}^{\bar{j}_{1}}-\frac{1}{N_{c}} \delta_{i_{1}}^{\bar{j}_{1}} \delta_{i_{2}}^{\bar{j}_{2}}
$$

In each diagram the color factor associated with each vertex is decomposed according to Eq. (105) and each internal gluon line color flow according to Eq. (106). It is very useful to employ diagrammatic techniques in order to follow the color flow, [64, 65]. These two substitutions discussed above, (105) and (106) are schematically illustrated in Fig. 3 and 4. An example of these substitutions is given in Fig. 5 where a 5-point amplitude is considered, see for example [44].

Eq. (106) can be interpreted as the statement that the $S U\left(N_{c}\right)$ generators are $N_{c} \times N_{c}$ hermitian, traceless matrices. The term proportional to $1 / N_{c}$ ensures that the trace vanishes. One can introduce the auxiliary generator of the $U(1)$ group $\left(T_{U(1)}^{a}\right)_{i}^{\bar{j}}=\frac{1}{\sqrt{N_{c}}} \delta_{i}^{\bar{j}}$ which commutes with $S U\left(N_{c}\right)$ and thus 

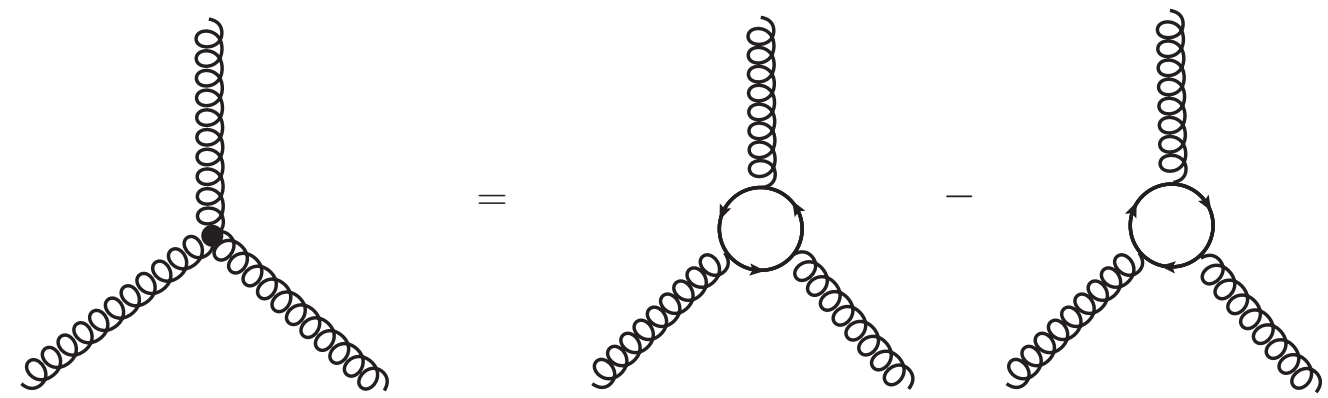

Figure 3: Diagrammatic expression for the color identity Eq. (105). Graph on the left side represents color structure constant $f^{a b c}$, 'gluon lines' represent the adjoint indices, single 'quark-like' line denotes the indices of the fundamental representation. The coupling of the 'gluon' and 'quark' lines denotes the generator $T^{a}$. The different orientations of the 'quark' line represent different orderings of $T^{a}$ matrices in Eq. (105).

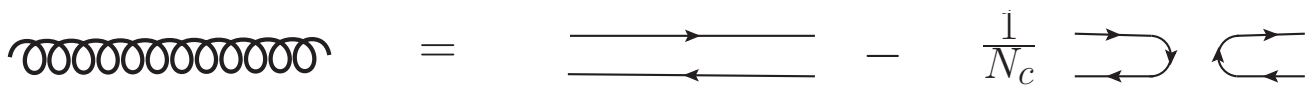

Figure 4: Diagrammatic expression for the color identity Eq. (106).

can be referred to as a 'photon', see for example [44]. While performing the color decomposition using Eqs. (105) and (106) the term $1 / N_{c}$ will not contribute there, which is the reflection of the fact that the auxiliary 'photon' field does not couple to gluons (this will change if the external quarks are present see $[44,61])$. The final diagram in Fig. 5 with a single 'quark' line and 5 gluons attached to it is a pictorial representation of the term containing single color trace of 5 matrices

$$
\operatorname{Tr}\left(T^{a_{1}} T^{a_{2}} T^{a_{3}} T^{a_{4}} T^{a_{5}}\right),
$$

Therefore any tree diagram for the $n$-gluon scattering can be reduced to a sum of the terms which contain single traces of color matrices. This decomposition can be summarized in the following relation

$$
\mathcal{A}_{n}=\sum_{\{1,2, \ldots, n\}^{\prime}} \operatorname{Tr}\left(T^{a_{1}} T^{a_{2}} \ldots T^{a_{n}}\right) \mathcal{M}\left(1^{\lambda_{1}} 2^{\lambda_{2}} \ldots n^{\lambda_{n}}\right)
$$

where the sum is over all non-cyclic permutations of $1,2, \ldots, n$ and $\lambda_{i}$ 's are polarizations of the gluons. Expression Eq. (108) provides gauge invariant decomposition of the QCD amplitude. Partial amplitudes $\mathcal{M}$ are still very complicated but significantly simpler than the full amplitudes $\mathcal{A}_{n}$. They contain information about the kinematical parts of the amplitude. It turns out that, because of the cyclic ordering of gluons the singularities of the partial amplitudes occur only in a limited set of the momentum channels, which correspond to the sums of the cyclically adjacent particle momenta. That is for example a 5 -point amplitude $\mathcal{M}\left(1^{\lambda_{1}}, 2^{\lambda_{2}}, 3^{\lambda_{3}}, 4^{\lambda_{4}}, 5^{\lambda_{5}}\right)$ will have singularities in $s_{12}, s_{23}, s_{34}, s_{45}, s_{51}$ where $s_{i j}=$ $\left(k_{i}+k_{j}\right)^{2}$ is the Mandelstam variable. On the contrary, it will not have singularities in $s_{13}, s_{24}, s_{35}, s_{41}, s_{52}$ channels. This feature of the color-ordered amplitudes will become evident once we study Parke-Taylor amplitudes in the following subsections.

The color decomposition can be generalized beyond tree level to the loop calculation [63], the difference being that the decomposition becomes more complicated since it involves single and double trace terms.

The subamplitudes $\mathcal{M}(1,2,3, \ldots, n)$ possess number of important properties [43] :

- $\mathcal{M}(1,2,3, \ldots, n)$ is gauge invariant

- $\mathcal{M}(1,2,3, \ldots, n)$ is invariant under cyclic permutations of $1,2, \ldots, n$ 


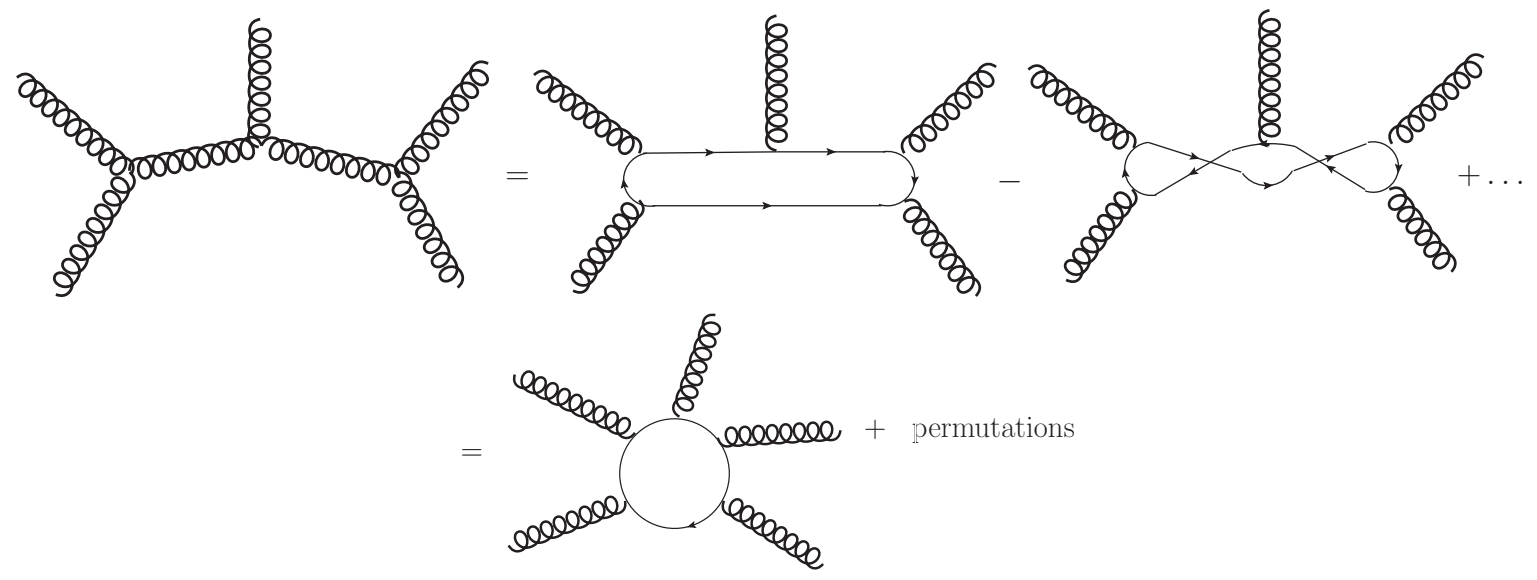

Figure 5: Diagrammatic expression for the color reduction of the 5-point amplitude to the single trace. It is understood that the last diagram contains also all the permutations of color indices.

- $\mathcal{M}(n, n-1, \ldots, 2,1)=(-1)^{n} \mathcal{M}(1,2, \ldots, n)$

- They satisfy Dual Ward Identity or photon decoupling equation

$$
\mathcal{M}(1,2, \ldots, n)+\mathcal{M}(2,1, \ldots, n)+\mathcal{M}(2,3,1, \ldots, n)+\cdots+\mathcal{M}(2,3, \ldots, 1, n)=0
$$

- Factorization of $\mathcal{M}(1,2, \ldots n)$ on multi-gluon poles

- Incoherence to leading order in the number of colors

$$
\sum_{\text {colors }}\left|\mathcal{A}_{n}\right|^{2}=N_{c}^{2}\left(N_{c}^{2}-1\right) \sum_{\{1,2, \ldots, n\}^{\prime}}\left[|\mathcal{M}(1,2, \ldots, n)|^{2}+\mathcal{O}\left(1 / N_{c}^{2}\right)\right] .
$$

\subsection{Tree level examples}

In this subsection we shall compute a few tree level examples for the gluonic amplitudes, to demonstrate the helicity formalism at work. Let us first start by considering the color-ordered amplitude with only (massless) gluons, $\mathcal{M}_{n}\left(1^{\lambda_{1}}, 2^{\lambda_{2}}, \ldots, n^{\lambda_{n}}\right)$, where $n$ denotes the number of external legs and $\lambda_{k}$ are the helicities of the gluons. Gluon labeled $i$ has momentum $k_{i}$ and corresponding helicity $\lambda_{i}$. In the following we shall use the standard convention used in the literature, that is we treat all the gluons as outgoing [43].

Consider first the amplitude in which all the outgoing gluons have the same helicities, i.e.

$$
\mathcal{M}_{n}\left(1^{ \pm}, 2^{ \pm}, \ldots, n^{ \pm}\right) .
$$

It is easy to see that, each term in this amplitude will have factor $\varepsilon_{i}^{ \pm}\left(q_{i}\right) \cdot \varepsilon_{j}^{ \pm}\left(q_{j}\right)$. Now, if we take all the reference momenta to be equal to $q$, then this amplitude will vanish automatically since $\varepsilon_{i}^{ \pm}(q) \cdot \varepsilon_{j}^{ \pm}(q)=0$. In fact it is sufficient to make only one such term to be equal to zero, and such choice of the reference momentum always exists. We shall see on the light-front that the reference momentum is identical for all the particles and therefore the vanishing condition is easily satisfied for this choice of polarizations. As a consequence the amplitudes in which all the gluons have the same helicities vanish for any number of external legs.

Now let us consider the amplitude in which one gluon has opposite helicity to the rest. Due to the cyclic property of the amplitudes, without loosing any generality one can choose this gluon to be the first one. Therefore we have

$$
\mathcal{M}_{n}\left(1^{\mp}, 2^{ \pm}, \ldots, n^{ \pm}\right)
$$


One can show that this amplitude vanishes as well by choosing the following reference momenta:

$$
q_{1}=k_{n}, \quad q_{2}=q_{3}=\cdots=q_{n}=k_{1} .
$$

In this case we shall have that

$$
\varepsilon_{1}^{ \pm}\left(k_{n}\right) \cdot \varepsilon_{i \neq 1}^{ \pm}\left(k_{1}\right)=0, \quad \varepsilon_{i}^{ \pm}\left(k_{1}\right) \cdot \varepsilon_{j}^{ \pm}\left(k_{1}\right)=0 \quad \text { for } \quad i, j \neq 1
$$

Thus we have that

$$
\mathcal{M}_{n}\left(1^{\mp}, 2^{ \pm}, \ldots, n^{ \pm}\right)=0, \quad n>3 .
$$

The amplitude for $n=3$ does not vanish though. This is because one cannot choose the reference momenta to be equal to the external momenta since the polarization vectors would become singular due to the energy-momentum conservation condition which gives $k_{i} \cdot k_{j}=0$.

The vanishing properties of the scattering amplitudes in these particular helicity configurations can be also proven using supersymmetry relations [66, 67].

Let us now evaluate the amplitude with two gluons of opposite helicity to the rest. It will turn out that it is non-vanishing

$$
\mathcal{M}_{4}\left(1^{-}, 2^{-}, 3^{+}, 4^{+}\right) \text {. }
$$

To compute this amplitude, one can choose the gluon momenta as the reference momenta for the polarization vectors. To this aim let us choose $q_{1}=q_{2}=k_{4}$ and $q_{3}=q_{4}=k_{1}$. It is easy to convince ourselves that the only non-vanishing contribution comes from the contraction $\varepsilon_{2}^{-} \cdot \varepsilon_{3}^{+} \neq 0$. There are three diagrams, but only one contributes here with this choice of the polarization vectors and it is the diagram with the gluon exchanged in the $s_{12}$ channel. The contribution from this graph is therefore

$$
\mathcal{M}_{4}\left(1^{-}, 2^{-}, 3^{+}, 4^{+}\right)=-\frac{2 i g^{2}}{s_{12}}\left(\varepsilon_{2}^{-} \cdot \varepsilon_{3}^{+}\right)\left(\varepsilon_{1}^{-} \cdot k_{2}\right)\left(\varepsilon_{4}^{+} \cdot k_{3}\right)=-i g^{2} \frac{\langle 12\rangle[34]^{2}}{[12]\langle 14\rangle[14]} .
$$

Using various relations, like antisymmetry of spinor products, momentum conservation one can finally cast the result into a very compact and appealing form

$$
\mathcal{M}_{4}\left(1^{-}, 2^{-}, 3^{+}, 4^{+}\right)=i g^{2} \frac{\langle 12\rangle^{4}}{\langle 12\rangle\langle 23\rangle\langle 34\rangle\langle 41\rangle} .
$$

It turns out that all other amplitudes, except one, with the same configuration of helicities but for different gluons, can be obtained using parity or cyclic symmetry. The only other amplitude is

$$
\mathcal{M}_{4}\left(1^{-}, 2^{+}, 3^{-}, 4^{+}\right)=i g^{2} \frac{\langle 13\rangle^{4}}{\langle 12\rangle\langle 23\rangle\langle 34\rangle\langle 41\rangle} .
$$

and can be obtained from the decoupling identity

$$
\mathcal{M}_{4}\left(1^{-}, 2^{+}, 3^{-}, 4^{+}\right)+\mathcal{M}_{4}\left(1^{-}, 3^{-}, 2^{+}, 4^{+}\right)+\mathcal{M}_{4}\left(1^{-}, 3^{-}, 4^{+}, 2^{+}\right)=0 .
$$

We observe that in general one can write the amplitude for this configuration of helicities as

$$
\mathcal{M}_{4}=i g^{2} \frac{\langle i j\rangle^{4}}{\langle 12\rangle\langle 23\rangle\langle 34\rangle\langle 41\rangle} .
$$

where $i, j$ are the gluons with negative helicities.

It can be shown that the above formula generalizes to amplitudes for arbitrary number of legs in which two gluons have helicities different than the rest. The amplitudes for these helicity configurations have particularly simple form known as Parke-Taylor formula [19]

$$
\mathcal{M}_{n}\left(1^{-}, 2^{-}, 3^{+}, \ldots, n^{+}\right)=i g^{n-2} \frac{\langle 12\rangle^{4}}{\langle 12\rangle\langle 23\rangle \ldots\langle n 1\rangle} .
$$

They are referred to as Maximally Helicity Violating (MHV) amplitudes, and they were conjectured in [19]. Their form was proven rigorously in [56] using the recursion relations which we shall discuss in the next section. 


\subsection{Berends-Giele recursion relations for off-shell currents}

Using the above helicity rules one can compute amplitudes with more number of legs and for the arbitrary helicity configurations. The calculation quickly becomes rather complicated though as the number of graphs which need to be taken into account becomes very large with an increasing number of legs. In order to simplify the calculation, one can use recursive techniques. The idea being, that one defines a building block, an off-shell current with one particle off-shell while the rest are on-shell. Once this is defined, one can construct the current with higher number of legs using the currents with smaller number of legs and appropriate vertices. By putting the off-shell particle on mass shell one obtains the on-shell scattering amplitude.

In some cases these recursion relations can be solved exactly. In general, however, this may not be possible. Nevertheless these recursion relations can be implemented into the computer code to obtain the amplitudes up to the desired number of external legs.

In order to set up the recursion relation one constructs an object with $n+1$ legs, in which all legs but one are on-shell. Momentum conservation is still satisfied for this quantity. This object is an off-shell current and is denoted by

$$
J_{\mu}(1,2, \ldots, n),
$$

where $1,2, \ldots, n$ are on-shell legs and for the pure gluon case it is depicted in Fig. 6.

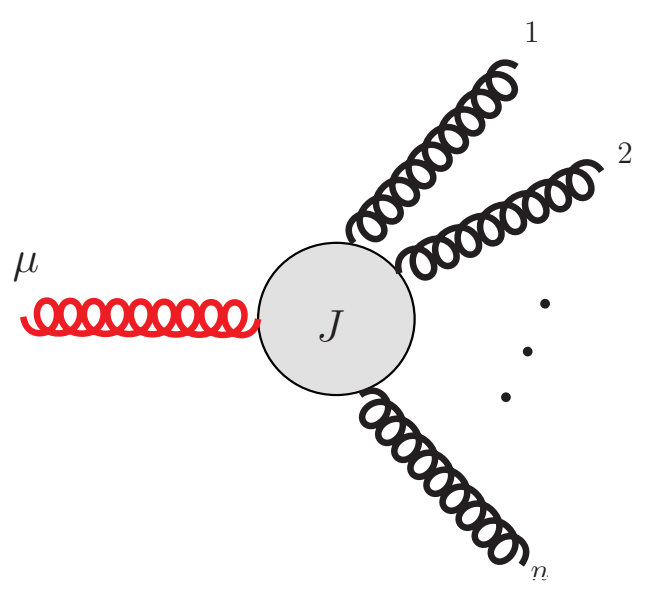

Figure 6: Color-ordered gluonic off-shell current $J^{\mu}(1, \ldots, n)$. The red colored gluon leg labeled by $\mu$ is off-shell, the rest of the legs (in black) denoted by 1 to $n$ are on-shell.

This off-shell current can be obtained by summing the color ordered Feynman graphs. The uncontracted leg is denoted by $\mu$ and the off-shell propagator is included in $J_{\mu}$. The on-shell subamplitude can be obtained from this current by multiplying by the inverse propagator, allowing the momentum to be on-shell and contracting with the appropriate polarization vector

$$
\mathcal{M}_{n+1}(1,2, \ldots, n+1)=\left.\left[i k_{1 \ldots n}^{2} \varepsilon^{\mu}\left(k_{n+1}\right) J_{\mu}(1, \ldots, n)\right]\right|_{k_{1 \ldots n}=-k_{n+1}},
$$

where we have defined handy notation which will be used later on extensively

$$
k_{1 \ldots n} \equiv \sum_{i=1}^{n} k_{n} .
$$

Since these currents are off-shell they are also (in principle) gauge dependent. Therefore they depend on the reference momenta for the on-shell gluons which must be kept fixed in the calculation. The gauge 
invariance is then restored after the amplitude is extracted and the remaining off-shell gluon is put on-shell. Nevertheless, these currents can be used as building blocks to obtain gauge invariant on-shell amplitudes. Quark off-shell currents can also be constructed in addition to gluon currents, see [20].

In order to construct the recursion, one considers first an off-shell gluonic current with one off-shell leg and $n$ on-mass shell legs. The off-shell leg has to be connected to the rest of the diagram either through the 3-gluon or 4-gluon vertex. At these vertices the other legs need to be connected to other gluonic currents with fewer number of legs. This can be diagrammatically illustrated in Fig. 7.

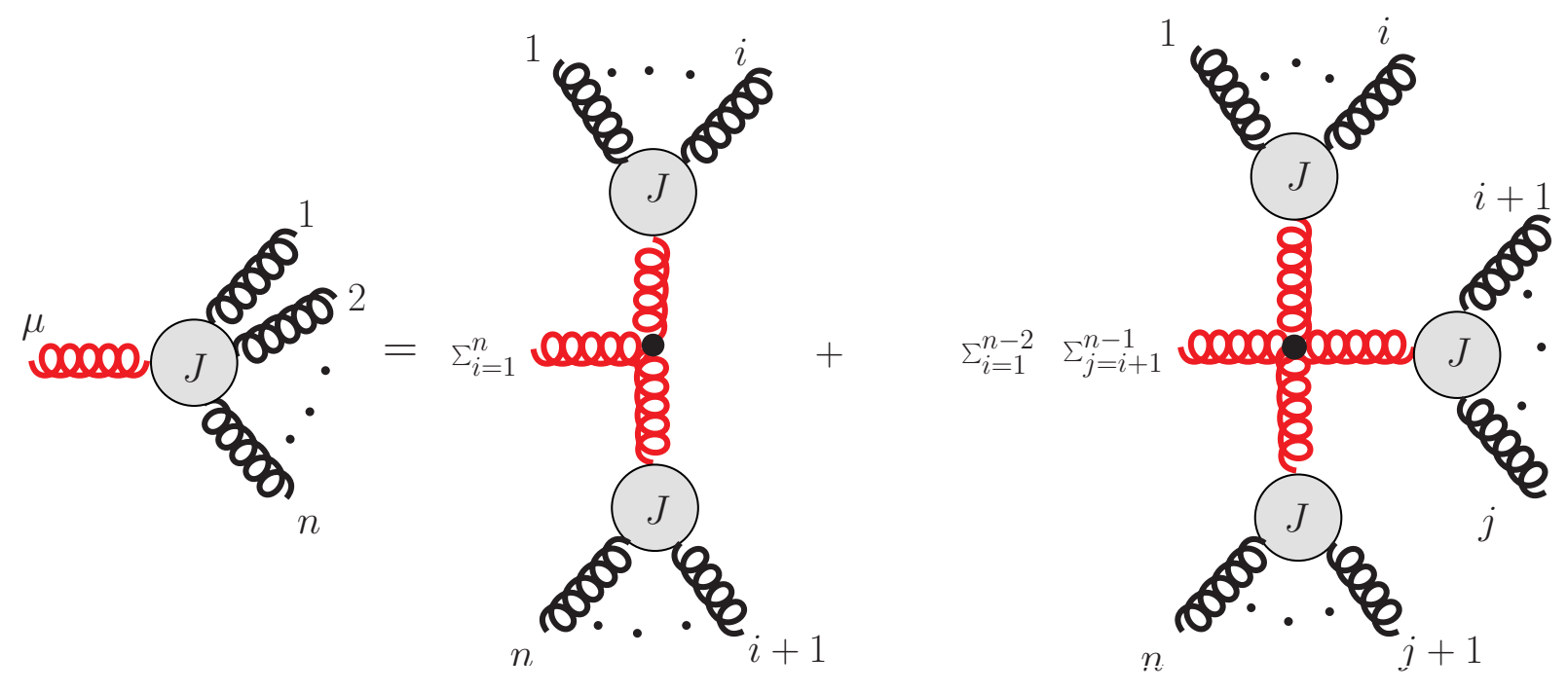

Figure 7: Diagrammatic expression for the Berends-Giele recursion relation Eq. (124) for the off-shell current $J^{\mu}$. Red lines indicate off-shell gluons and black lines on-shell final state gluons.

The expression for this recursion can be written as

$$
\begin{array}{r}
J^{\mu}(1, \ldots, n)=\frac{-i}{k_{1 \ldots n}^{2}}\left[\sum_{i=1}^{n-1} V_{3}^{\mu \nu \rho}\left(k_{1 \ldots i}, k_{i+1 \ldots n}\right) J_{\nu}(1, \ldots, i) J_{\rho}(i+1, \ldots, n)\right. \\
\left.+\sum_{i=1}^{n-2} \sum_{j=i+1}^{n-1} V_{4}^{\mu \nu \rho \sigma} J_{\nu}(1, \ldots, i) J_{\rho}(i+1, \ldots, j) J_{\sigma}(j+1, \ldots, n)\right],
\end{array}
$$

where $V_{3}^{\mu \nu \rho}$ and $V_{4}^{\mu \nu \rho \sigma}$ are three- and four-gluon color ordered vertices. These currents satisfy several important properties:

- The decoupling relation

$$
J^{\mu}(1,2, \ldots, n)+J^{\mu}(2,1,3, \ldots, n)+\cdots+J^{\mu}(2,3, \ldots, n, 1)=0,
$$

- The reflection identity

$$
J^{\mu}(1,2,3, \ldots, n)=(-1)^{n+1} J^{\mu}(n, \ldots, 3,2,1),
$$

- Current conservation

$$
k_{1 \ldots n}^{\mu} J_{\mu}(1,2, \ldots, n) .
$$

The analytical expressions for the currents can be very complicated but they simplify for some specific configurations of helicities. In particular, the forms of these currents are quite compact if all 
the helicities are the same or if one is different than the others. In the case when all of the outgoing helicities are the same, the current has the form [20]

$$
J_{\mu}\left(1^{+}, 2^{+}, \ldots, n^{+}\right)=g^{n-1} \frac{\left\langle q-\left|\gamma^{\mu} \not k_{1 \ldots n}\right| q+\right\rangle}{\sqrt{2}\langle q 1\rangle\langle 12\rangle \ldots\langle n-1 n\rangle\langle n q\rangle},
$$

and where $q$ is the common reference momentum for all the gluons. For the case when one gluon has a negative helicity the expression is little bit more complicated but also quite compact, see for example $[44]$

$$
J_{\mu}\left(1^{-}, 2^{+}, \ldots, n^{+}\right)=g^{n-1} \frac{\left\langle 1-\left|\gamma^{\mu} \not k_{2 \ldots n}\right| 1+\right\rangle}{\sqrt{2}\langle 12\rangle \ldots\langle n-1 n\rangle\langle n 1\rangle} \sum_{m=3}^{n} \frac{\left\langle 1-\left|\not k_{m} \not k_{1 \ldots m}\right| 1+\right\rangle}{k_{1 \ldots m-1}^{2} k_{1 \ldots m}^{2}} .
$$

The Parke-Taylor amplitude of Eq. (120) can be obtained by contracting the current $J^{\mu}$ with the appropriate polarization vector, amputating the propagator and putting the leg on-shell. In addition, one can easily check that the vanishing conditions for on-shell amplitudes in Eq. (113) are satisfied with these forms of the currents. In the case of the current given in Eq. (125), the resulting on-shell amplitude will vanish for both helicities of the gluon $n+1$. This is evident from expression Eq. (125) since there is no pole in $P_{1, n}^{2}=k_{n+1}^{2}$ and so the amplitude after amputating this propagator and putting it on-shell will vanish for both choices of the helicities of the gluon $n+1$, i.e. $\mathcal{M}( \pm,+, \ldots,+)=0$ consistent with Eq. (113). For the case of the current given in Eq. (126) the requirement from the pole will select one term from the sum, the one for which $m=n$. In this case however, the non-vanishing contribution will be only for choice of the polarization vector $\varepsilon_{n+1}^{-}$, this will give the Parke-Taylor amplitude $(-,-,+, \ldots,+)$. For the opposite choice of the polarization vector $\varepsilon_{n+1}^{+}$the contraction with the current vanishes, and thus the amplitude $(-,+, \ldots,+)$ vanishes too. This can be seen by using the reference vector $q_{n+1}=k_{1}$ and utilizing the fact that in this case $\langle 1-| \phi_{n+1}^{+}\left(k_{1}\right)=0$ according to the rules given in Eq. (102).

The Berends-Giele recursion relations Eq. (124) were also formulated in the light-cone gauge [57] and used to compute the amplitude for the $(-,-,-,+, \cdots+)$ configuration of helicities.

\subsection{BCFW recursion relations for tree-level amplitudes}

The Berends-Giele recursion relations discussed in the previous section allow to compute in general the amplitudes with any number of external legs. The potential difficulty in the application of this method is that it relies on the use of the gauge dependent off-shell amplitude, or current $J^{\mu}$. The final results are on-shell objects and are of course gauge independent as they should be. As we shall see later in Sec. 5.4, the use of off-shell amplitudes is not necessarily a drawback, since one can formulate gauge invariant extensions of the off-shell amplitudes and find the recurrence relations for them as well. In this section however, we shall recall another very powerful recursion relation that does not rely on the off-shell quantities but rather on the on-shell amplitudes which are analytically continued to the complex plane. This is the BCFW recursion relation which was originally derived by studying loop diagrams by Britto, Cachazo and Feng [21]. Later on, an elegant proof was given by Britto, Cachazo, Feng and Witten [22] without resorting to the loop amplitudes but rather by arguments that rely on the analytical structure of the scattering amplitudes. Excellent reviews on this topic exists, see for example [45, 68, 69], here we recall the fundamental arguments leading to the BCFW recursion following original presentation of the proof $[22]$.

A generic scattering amplitude considered here is the tree level gluon amplitude. Such an amplitude is a rational function of the external gluon momenta. This is evident, for example, from the structure of the Parke-Taylor amplitude presented in Eq. (120) which is a rational function of the spinor products. Such amplitude can be analytically continued into the complex momenta. In this case the amplitude becomes a meromorphic function of the complex variable which means it is analytic except for the 
isolated poles. The poles of the tree-level amplitudes arise only from the internal propagators in the Feynman diagrams.

Let us start from the general consideration of the complex deformation of the amplitude with $n$ number of external gluons. The idea is to pick two external gluons which can be labeled $i$ and $j$, and their momenta are labeled $k_{i}$ and $k_{j}$. These will be called reference momenta. The BCFW complex shift is then

$$
\begin{aligned}
\hat{k}_{i} \equiv k_{i}(z) & =k_{i}-z q, \quad \hat{k}_{j} \equiv k_{j}(z)=k_{j}+z q, \\
q^{2} & =0, \quad k_{i} \cdot q=k_{j} \cdot q=0,
\end{aligned}
$$

where $q^{\mu}$ is some auxiliary vector and $z$ is complex number. One can give a more physical picture of the direction of the auxiliary vector $q^{\mu}$, if one goes to the Lorentz frame where the spatial components of $k_{i}$ and $k_{j}$ are both in the $x_{3}$ direction. Then according to condition given by Eq. (127) the vector $q$ must be in the $\left(x_{1}, x_{2}\right)$ plane and must be complex. The rest of the external momenta are not shifted. The amplitude then becomes a meromorphic function of the complex variable $z$ and the only singularities are given by the poles originating from the internal propagators.

A particular realization of this shift is usually expressed in terms of the spinors. Below we shall use the original representation that was used in [22] in terms of the two-dimensional (Weyl spinors)

$$
u_{+}\left(k_{i}\right)=|i+\rangle \rightarrow \lambda_{i}^{\alpha}, \quad u_{-}\left(k_{i}\right)=|i-\rangle \rightarrow \tilde{\lambda}_{i}^{\dot{\alpha}},
$$

where $u_{ \pm},|k \pm\rangle$ are four-component spinors defined previously in Sec. 3 and $\lambda_{i}^{\alpha}$ and $\tilde{\lambda}_{i}^{\dot{\alpha}}(\alpha, \dot{\alpha}=1,2)$ are the two-component spinors. In terms of this notation the null vector $k_{i}$ can be expressed as a bi-spinor $k_{i}^{\alpha \dot{\alpha}}=\lambda_{i}^{\alpha} \tilde{\lambda}_{i}^{\dot{\alpha}}$ and the spinor products introduced before can be rewritten in a two-component version as

$$
\begin{aligned}
& \langle i j\rangle=\varepsilon_{\alpha \beta} \lambda_{i}^{\alpha} \lambda_{j}^{\beta}, \\
& {[i j]=\varepsilon_{\dot{\alpha} \dot{\beta}} \tilde{\lambda}_{i}^{\dot{\alpha}} \tilde{\lambda}_{j}^{\dot{\beta}},}
\end{aligned}
$$

where $\varepsilon_{\alpha \beta}, \varepsilon_{\dot{\alpha} \dot{\beta}}$ are antisymmetric tensors.

In terms of these two-dimensional spinors the complex shift of the momenta can be realized as follows [22]

$$
\begin{aligned}
& k_{i}(z)=\lambda_{i}\left(\tilde{\lambda}_{i}-z \tilde{\lambda}_{j}\right), \\
& k_{j}(z)=\left(\lambda_{j}+z \lambda_{i}\right) \tilde{\lambda}_{j},
\end{aligned}
$$

which in turn can be interpreted as the transformation of the spinors themselves

$$
\begin{aligned}
& \hat{\lambda}_{j} \equiv \lambda_{j}+z \lambda_{i}, \\
& \hat{\tilde{\lambda}}_{i} \equiv \tilde{\lambda}_{i}-z \tilde{\lambda}_{j} .
\end{aligned}
$$

Clearly, we see that this transformation leaves the sum of the shifted momenta independent of the complex variable $z$

$$
k_{i}(z)+k_{j}(z)=k_{i}+k_{j},
$$

and consequently the total momentum for this amplitude $\sum_{i} k_{i}$ is unaffected by the shift and still zero. One is ultimately interested in evaluating the scattering amplitude at $z=0$. Since the amplitude $\mathcal{A}(z)$ is a meromorphic function we can relate its value at $z=0$ to the sum over the residues at poles $z_{k}$. To be more specific, we can use the Cauchy theorem to write

$$
\mathcal{R}=\frac{1}{2 \pi i} \oint_{\mathcal{C}} \frac{\mathcal{A}(z)}{z} d z=\mathcal{A}(0)+\left.\sum_{k} \operatorname{Res}\left[\frac{\mathcal{A}(z)}{z}\right]\right|_{z=z_{k}},
$$


where $\mathcal{C}$ is the circle at infinity and $z_{k}$ are the locations of the singularities of the amplitude $\mathcal{A}$. If the amplitude $\mathcal{A}(z) \rightarrow 0$ when $|z| \rightarrow \infty$ then this contour integral vanishes, i.e. $\mathcal{R}=0$, and one can relate the value of the amplitude $\mathcal{A}(0)$ to the sum over the residues. The vanishing condition has been proved from Feynman diagrams [22] and also using background field method [70].

As mentioned earlier, the singularities at $z_{k}$ come from the propagators of the amplitude $\mathcal{A}$. In Yang-Mills theory at the tree level the propagator is the sum of the adjacent external momenta. We can thus write that the propagator takes the following form

$$
k_{a b}(z)=k_{a}(z)+k_{a+1}(z)+\cdots+k_{b}(z), \quad b>a,
$$

where $a, b \in\{1, \ldots, n\}$. For the choice of the shift considered here, the propagator is independent of $z$ if either: both of the gluon momenta $i, j$ are in the range from $a$ to $b$; or: none of them is in the range from $a$ to $b$. Therefore, without the loss of generality, one can assume that $j \in\{a, b\}$ and $i$ is not in this range, and in this case the propagator $\hat{k}_{a b}$ depends on $z$

$$
\hat{k}_{a b}(z)=k_{a b}+z \lambda_{i} \tilde{\lambda}_{j}=k_{a b}+z q,
$$

and therefore

$$
\hat{k}_{a b}^{2}(z)=k_{a b}^{2}+2 z q \cdot k_{a b},
$$

Therefore the propagator has now a simple pole located at

$$
z_{a b}=-\frac{k_{a b}^{2}}{2 q \cdot k_{a b}} .
$$

In order to evaluate the residue of the amplitude at the pole $z_{a b}$ one needs to know the behavior of the amplitude in the limit of the singular internal propagator. It is however known, see for example $[43,44]$, that the amplitude factorizes in this limit into two on-shell amplitudes. This factorization can be expressed as

$$
\mathcal{A}(1, \ldots, n) \stackrel{p_{a b}^{2} \rightarrow 0}{\sim} \sum_{\lambda} \mathcal{A}_{L}\left(-p_{a b}^{\lambda}\right) \frac{1}{p_{a b}^{2}} \mathcal{A}_{R}\left(p_{a b}^{-\lambda}\right)
$$

where $p_{a b}$ is the propagator that is singular and the sum goes over the $\lambda$, which is the helicity of the exchanged particle (the amplitudes $\mathcal{A}_{L}$ and $\mathcal{A}_{R}$ are on-shell). Above, we have suppressed other arguments for the sub amplitudes. Utilizing the factorization property one can find the residue at pole $z_{a b}$ to be

$$
-\left.\left.\sum_{\lambda} \mathcal{A}_{L}\left(a, \ldots b,-\hat{k}_{a b}^{\lambda}\right)\right|_{z=z_{a b}} \frac{1}{k_{a b}^{2}} \mathcal{A}_{R}\left(\hat{k}_{a b}^{-\lambda}, b+1, \ldots, a-1\right)\right|_{z=z_{a b}} .
$$

Summing over all the residues and using Eq. (133) together with the vanishing condition $\mathcal{R}=0$ one obtains the BCFW recursion formula

$$
\mathcal{A}(1, \ldots, n)=\left.\left.\sum_{a, b} \sum_{\lambda} \mathcal{A}_{L}\left(a, \ldots b,-\hat{k}_{a b}^{\lambda}\right)\right|_{z=z_{a b}} \frac{1}{k_{a b}^{2}} \mathcal{A}_{R}\left(\hat{k}_{a b}^{-\lambda}, b+1, \ldots, a-1\right)\right|_{z=z_{a b}},
$$

where the first sum is over all the decompositions such that the two reference (or shifted) momenta are separately in each of the subamplitudes. The second sum is performed over the helicity of the exchanged particle. This recursion is diagrammatically depicted in Fig. 8. Note that, the dependence of the sub amplitudes on the choice of the reference momentum, which is evident on the right-hand-side of the recursion, cancels out in the resulting amplitude on the left hand side.

We stress again that the subamplitudes are on-shell, but with the shifted momenta evaluated at the residue poles. The momentum of the connecting propagator is real however, and therefore different than the momentum which goes into the subamplitudes. Since the sub amplitudes are with lower number 


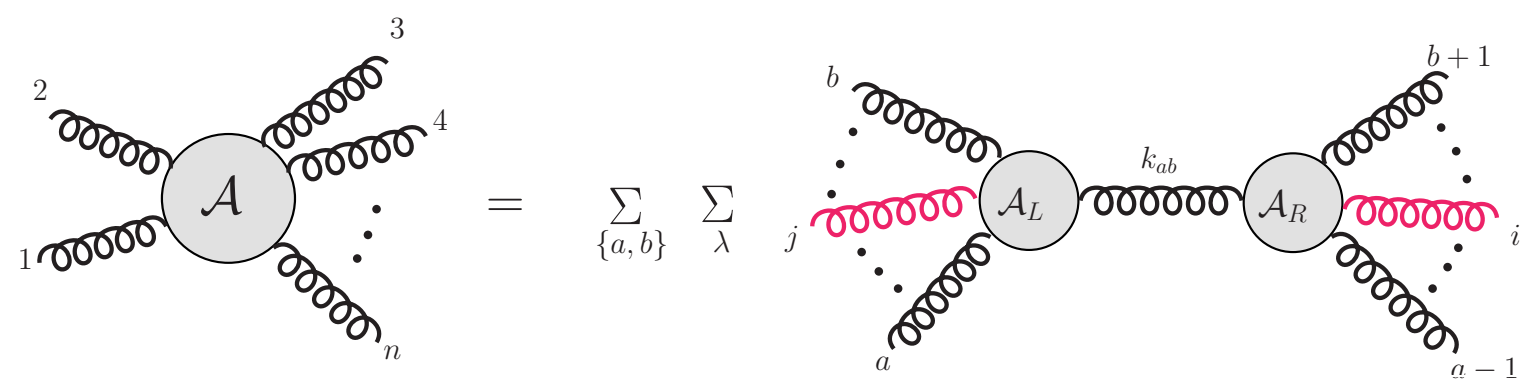

Figure 8: Schematic representation of the BCFW recursion relation in Eq. (140). The two gluons in red are the reference gluons. The first sum is over all the possible decompositions of cyclically ordered gluons with one reference gluon on each side. The second sum is over the helicities of the exchanged internal gluon.

of legs, this relation is indeed recursion relation that allows very efficient computation of the on-shell amplitudes with higher number of legs through the amplitudes with lower number of legs.

The BCFW recursion relation has been recently generalized for the off-shell scattering amplitudes [71-73]. As we shall see later, it is possible to construct the gauge invariant extension of the off-shell amplitudes, which satisfy the Ward identities with respect to on-shell legs. Obtaining the off-shell amplitudes is particularly important since they can be used in the framework of the $k_{T}$-factorization to describe various processes in high energy hadron-hadron and hadron-lepton scattering. However, the calculation can be very complicated for the off-shell case. The extended BCFW recursion derived in [71] is a very efficient tool, since it enables to compute the off-shell gauge invariant amplitudes via recursion in terms of the subamplitudes with lower number of legs. Since it makes use of the gauge invariant amplitudes it is different than the Berends-Giele recursion relation which operates on the gauge dependent off-shell currents. What is also interesting, and we shall see it explicitly in the case of the light front calculations, that the gauge invariant off-shell expressions constructed in such a way follow the same structure as the on-shell MHV scattering amplitudes (modulo some pre factor) when expressed in terms of the spinor products [71].

\section{Wave functions and fragmentation functions on the light- front}

In the previous section we have discussed the helicity amplitudes and the spinor formalism in QCD. These amplitudes were obtained using the standard Feynman rules of the QCD quantized at instant time. In this and the following sections we shall focus on the perturbative light-front theory. In particular, we shall introduce and discuss various objects which will be very useful in the construction of the scattering amplitudes on the light-front. The exact form for the $n$-component of the gluon wave function and for the fragmentation function in the special choice of the helicities, will be discussed. These two subamplitudes are off-shell objects, i.e. they include the denominators either in the last state (for the case of the gluon wave function or in the initial state, for the case of the fragmentation functions). Later on we shall see, that using these subamplitudes one can construct useful recursion relations which allow to find the off-shell as well as on-shell scattering amplitudes on the light-front. In this section we shall be closely following the derivation and conventions used in [29, 30], for more details we refer the interested reader to those works. 


\subsection{Wave functions on the light-front}

Light-front theory allows for the construction of the particularly convenient and intuitive framework for the wave functions of hadrons in QCD. This framework is based on the Fock state decomposition of the hadronic wave functions. To be precise this decomposition allows for the characterization of the hadron as being composed of different combination of the quarks and gluons at a given light-cone time. For example the pion state can be represented as

$$
|\pi\rangle=\sum_{q \bar{q}}|q \bar{q}\rangle \psi_{q \bar{q} / \pi}+\sum_{q \bar{q} g}|q \bar{q} g\rangle \psi_{q \bar{q} g / \pi}+\ldots
$$

or more precisely, for the pion with momentum $P^{+}, \vec{P}_{\perp}$ the decomposition is

$$
\left|\pi ; P^{+}, \vec{P}_{\perp}\right\rangle=\sum_{n, \lambda_{i}} \int \tilde{\prod} \frac{d x_{i} d^{2} \vec{k}_{\perp i}}{\sqrt{x_{i}}}\left|n ; x_{i}, \vec{k}_{\perp, i}, \lambda_{i}\right\rangle \psi_{n / \pi}\left(x_{i}, \vec{k}_{\perp, i}, \lambda_{i}\right) .
$$

In the above the sum is over the different Fock components and helicities, and the measures of integration are defined as

$$
\tilde{\prod}_{i} d x_{i}=\prod_{i} d x_{i} \delta\left(1-\sum_{i} x_{i}\right), \quad \tilde{\prod}_{i} d^{2} \vec{k}_{\perp, i}=\prod_{i} d^{2} \vec{k}_{\perp, i} \delta^{2}\left(\sum_{j} \vec{k}_{\perp, j}\right)
$$

The wave function $\psi_{n / \pi}\left(x_{i}, \vec{k}_{\perp, i}, \lambda_{i}\right)$ is the amplitude for the $n$-parton component in the pion. In this section we shall first consider the wave function for the on-shell gluon, by which we mean an off-shell object with one incoming gluon on-shell and the rest, outgoing particles off-shell. Our goal is to find the explicit expressions for these $n$-particle components using the light-front perturbation theory (restricted to the tree-level approximation).

Let us consider a process, which involves only gauge fields, and which is illustrated schematically in Fig. 9. It is of course understood that all the vertices can occur in such object, that is 3, 4-gluon and Coulomb interactions. The incoming gluon has the four momentum $P^{\mu}$, and the color index $a$, and through subsequent splitting it evolves into a state containing $n$ gluons with momenta $\left(k_{1}, \ldots k_{n}\right)$ and color indices $\left(a_{1}, a_{2}, \ldots, a_{n}\right)$ correspondingly. Since we are computing this process in the light-front theory, the diagram shown is such that the light-front time $x^{+}$flows from the left to the right of this graph. The momentum of the incoming off-shell gluon with virtuality $-Q^{2}$ and a transverse momentum $\vec{q}_{\perp}$ can be written in the light-front variables,

$$
P^{\mu}=\left(z_{0} P^{+}, \vec{q}_{\perp}, \frac{-Q^{2}+\vec{q}_{\perp}^{2}}{z_{0} P^{+}}\right),
$$

with $P^{ \pm}=P^{0} \pm P^{3}$ as defined previously in Sec. 2.5. Here $z_{0}$ is the longitudinal momentum fraction carried by the incoming gluon, and obviously it is equal to unity in this case. In general, the longitudinal momentum fractions of all the particles are defined as

$$
z_{i}=\frac{k_{i}^{+}}{P^{+}}, \quad 0<z_{i} \leq 1,
$$

and note that they are positive according to the light-front rules, which were introduced previously in Sec. 2.5 .

The initial gluon then can split many times, with splittings being ordered in the light-front time $x^{+}$, and eventually evolves into a state of $n$ off-shell gluons, as shown in Fig. 9. The momenta of the gluons in the last state are given by

$$
k_{i}^{\mu}=\left(z_{i} P^{+}, \vec{k}_{i \perp}, k_{i}^{-}\right) .
$$


Here $\vec{k}_{i \perp}$ is the transverse component of the gluon momentum and the light-front energy $k_{i}^{-}$of the gluon $i$ is equal to

$$
k_{i}^{-}=\frac{\vec{k}_{i \perp}^{2}}{z_{i} P^{+}} .
$$

In Sec. 2 we have discussed general rules of the light-front perturbation theory, which were developed both in the case of scalar theory as well as in the case of gauge theory [9, 36, 38, 41]. Let us therefore recall that, in order to evaluate the contributions from the diagram depicted in Fig. 9 one needs to include the energy denominators for each of the intermediate states. The energy denominators in the light-front theory play the role of the covariant propagators in the standard formulation. On the light-front they are defined as the difference between the light-front energies of the initial and the intermediate state which is being considered. Recall that, in the light-front formulation the energies (or minus components) are not conserved in the intermediate states inside the denominators. This is actually a subtle issue, which will be further discussed when analyzing Ward identities on the light front, see Sec. 5.6. The energy denominators for the intermediate states are denoted as vertical dashed lines in the light-front diagrams. In the process depicted in Fig. 9 the last shown state with $n$ gluons is taken to be off-shell and therefore it includes the energy denominator which in this case is equal to

$$
D_{n}=P^{-}-\sum_{i=1}^{n} k_{i}^{-}=-\frac{1}{P^{+}}\left(\frac{Q^{2}}{z_{0}}-\frac{\vec{q}_{\perp}^{2}}{z_{0}}+\frac{\vec{k}_{1 \perp}^{2}}{z_{1}}+\frac{\vec{k}_{2 \perp}^{2}}{z_{2}}+\ldots+\frac{\vec{k}_{n \perp}^{2}}{z_{n}}\right) .
$$

Since $P^{+}$is the total momentum and all the results are boost invariant we shall use the convention in which we set it $P^{+}=1$. In general the expression for the wave function, which will be denoted by $\Psi_{n}$,

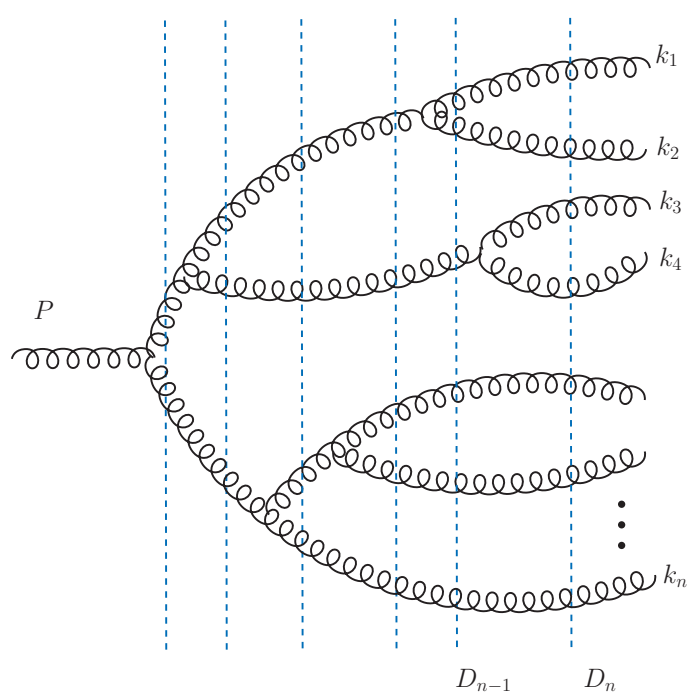

Figure 9: The light-front diagram contributing to the multi-gluon wave function of the incoming gluon with momentum $P$. The incoming (leftmost) gluon is on-shell, all the outgoing (rightmost) gluons are off-shell. The light-front time $x^{+}$flows from left to the right in this diagram. The vertical dashed lines symbolize different intermediate states where we need to evaluate the energy denominators. It is understood that the wave function scatters finally on some target.

the example of which is depicted in Fig. 9, with $n$ gluon-component can be schematically written as

$$
\Psi_{n} \sim g^{n-1} \prod_{j=1}^{n-1} \frac{V_{j}}{z_{j} D_{j}}
$$

where $V_{j}$ denote the vertices in the wave function. 


\begin{tabular}{|c|c|c|c|}
\hline$V\left(3^{+} \rightarrow 1^{+} 2^{+}\right)$ & $2 i g k_{3}^{+}\left(\frac{\varepsilon_{\perp}^{+} \cdot k_{2 \perp}}{k_{2}^{+}}-\frac{\varepsilon_{\perp}^{+} \cdot k_{1 \perp}}{k_{1}^{+}}\right)$ & $V_{4}\left(4^{+} \rightarrow 1^{-} 2^{+} 3^{+}\right)$ & $i g^{2}$ \\
\hline$V\left(3^{+} \rightarrow 1^{+} 2^{-}\right)$ & $2 i g k_{1}^{+}\left(\frac{\varepsilon_{\perp}^{-} \cdot k_{3 \perp}}{k_{3}^{+}}-\frac{\varepsilon_{\perp}^{-} \cdot k_{2 \perp}}{k_{2}^{+}}\right)$ & $V_{4}\left(4^{+} \rightarrow 1^{+} 2^{-} 3^{+}\right)$ & $-2 i g^{2}$ \\
\hline$V\left(3^{+} \rightarrow 1^{-} 2^{+}\right)$ & $2 i g k_{2}^{+}\left(\frac{\varepsilon_{\perp}^{-} \cdot k_{3 \perp}}{k_{3}^{+}}-\frac{\varepsilon_{\perp}^{-} \cdot k_{1 \perp}}{k_{1}^{+}}\right)$ & $V_{4}\left(4^{+} \rightarrow 1^{+} 2^{+} 3^{-}\right)$ & $i g^{2}$ \\
\hline$V\left(3^{-} \rightarrow 1^{-} 2^{-}\right)$ & $2 i g k_{3}^{+}\left(\frac{\varepsilon_{\perp}^{-} \cdot k_{2 \perp}}{k_{2}^{+}}-\frac{\varepsilon_{\perp}^{-} \cdot k_{1 \perp}}{k_{1}^{+}}\right)$ & $V_{4}\left(4^{-} \rightarrow 1^{+} 2^{-} 3^{-}\right)$ & $i g^{2}$ \\
\hline$V\left(3^{-} \rightarrow 1^{-} 2^{+}\right)$ & $2 i g k_{1}^{+}\left(\frac{\varepsilon_{\perp}^{+} \cdot k_{3 \perp}}{k_{3}^{+}}-\frac{\varepsilon_{\perp}^{+} \cdot k_{2 \perp}}{k_{2}^{+}}\right)$ & $V_{4}\left(4^{-} \rightarrow 1^{-} 2^{+} 3^{-}\right)$ & $-2 i g^{2}$ \\
\hline$V\left(3^{-} \rightarrow 1^{+} 2^{-}\right)$ & $2 i g k_{2}^{+}\left(\frac{\varepsilon_{\perp}^{+} \cdot k_{3 \perp}}{k_{3}^{+}}-\frac{\varepsilon_{\perp}^{+} \cdot k_{1 \perp}}{k_{1}^{+}}\right)$ & $V_{4}\left(4^{-} \rightarrow 1^{-} 2^{-} 3^{+}\right)$ & $i g^{2}$ \\
\hline$V\left(3^{+} \rightarrow 1^{-} 2^{-}\right)$ & 0 & $V_{4}\left(4^{+} \rightarrow 1^{+} 2^{+} 3^{+}\right)=V_{4}\left(4^{-} \rightarrow 1^{-} 2^{-} 3^{-}\right)$ & 0 \\
\hline \multirow[t]{3}{*}{$V\left(3^{-} \rightarrow 1^{+} 2^{+}\right)$} & 0 & $V_{4}\left(4^{+} \rightarrow 1^{-} 2^{-} 3^{+}\right)=V_{4}\left(4^{-} \rightarrow 1^{+} 2^{+} 3^{-}\right)$ & 0 \\
\hline & & $V_{4}\left(4^{+} \rightarrow 1^{-} 2^{+} 3^{-}\right)=V_{4}\left(4^{-} \rightarrow 1^{+} 2^{-} 3^{+}\right)$ & 0 \\
\hline & & $V_{4}\left(4^{+} \rightarrow 1^{+} 2^{-} 3^{-}\right)=V_{4}\left(4^{-} \rightarrow 1^{-} 2^{+} 3^{+}\right)$ & 0 \\
\hline$\lambda_{1}=-\lambda_{2}$ and $\lambda_{3}=\lambda_{4}$ & & $\lambda_{1}=\lambda_{2}$ or $\lambda_{3}=-\lambda_{4}$ & \\
\hline$V_{\text {inst }}\left(4^{\lambda_{4}} \rightarrow 1^{\lambda_{1}} 2^{\lambda_{2}} 3^{\lambda_{3}}\right)$ & $i g^{2} \frac{\left(k_{2}^{+}-k_{1}^{+}\right)\left(k_{3}^{+}+k_{4}^{+}\right)}{\left(k_{4}^{+}-k_{3}^{+}\right)^{2}}$ & $V_{i n s t}\left(4^{\lambda_{4}} \rightarrow 1^{\lambda_{1}} 2^{\lambda_{2}} 3^{\lambda_{3}}\right)$ & 0 \\
\hline
\end{tabular}

Table 2: Three, four and instantaneous gluon vertices projected onto the transverse polarization vectors. In the triple gluon vertex, gluon 3 is incoming and 1 and 2 outgoing. In the 4-gluon and instantaneous vertex gluon 4 is incoming and gluons 1,2,3 are outgoing. Other combinations can be easily found from inverting the momentum flow in the vertices. The convention used here differs by $1 / \sqrt{2}$ factor as compared with [43]. Some examples of these vertices are shown in Fig. 10.

In order to compute the gluon wave function for the specific configuration of the helicities it will be of importance to setup the recurrence relation in which the gluon wave functions for arbitrary number of external legs can be found from the gluon wave functions with the lower number of external legs. To further facilitate the computations we shall be using color ordered objects and with fixed configuration of helicities. Later on this will turn out to be useful in the computation of the scattering helicity amplitudes. Gluon wave function can be very complicated for the arbitrary helicity configurations but it turns out that they have particularly simple form if one chooses a specific configuration of the helicities.

In order to evaluate the gluon wave functions, we shall need the triple gluon and quartic vertices projected onto different helicity states. In Sec. 3 we have listed Feynman rules for the color ordered case. Taking the vertices and contracting them with the polarization vectors $\varepsilon_{\mu}^{\lambda}(k)$ defined in Eqs. (53) 
and (54) one can find the expressions which are listed in Tables 2. The example of these projections are also shown in Fig. 10 where again it is understood that the light-front time flows from left to right. Here, the convention is such that the first gluon is incoming and the rest of the gluons are outgoing. For now we shall be interested in specific configurations of the helicities which will make the computation of the wave functions easier. Namely, if the incoming gluon has the positive helicity and all the outgoing gluons have positive helicities then the only vertices that can appear in the gluon wave function are triple gluon vertices with the transitions $+\rightarrow++$. This stems from the fact that if there would be a transition into a " - " helicity state, then in order to have the final state with all " + " components there would have to be a transition $-\rightarrow++$ which however vanishes, see Table 2. Similarly, four gluon vertices and also Coulomb interactions will not give contributions to this configuration of helicities as they vanish when contracted with the appropriate polarization vectors, as can be seen from Table 2 .
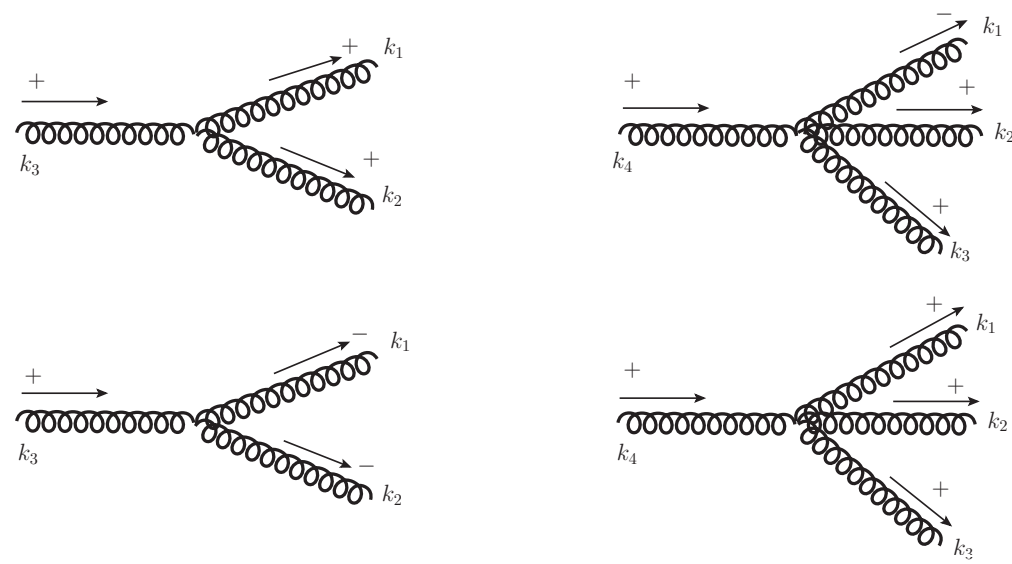

Figure 10: Example of three and four-gluon vertices in light front ordering with specified helicities. Top two vertices are non-vanishing and the bottom two are vanishing. The light-front time is flowing from left to right.

We observe that all the three-gluon vertices are proportional to the special combinations of the longitudinal and transverse variables. It is thus useful to introduce the following variable which will be used in the calculations on the light-front

$$
\vec{v}_{i j} \equiv\left(\frac{\vec{k}_{i \perp}}{z_{i}}-\frac{\vec{k}_{j \perp}}{z_{j}}\right) .
$$

and its complex projection

$$
v_{i j}=\vec{\varepsilon}_{\perp}^{-} \cdot \vec{v}_{i j}, \quad v_{i j}^{*}=\vec{\varepsilon}_{\perp}^{+} \cdot \vec{v}_{i j} .
$$

Variable $\vec{v}_{i j}$ has the interpretation of being the relative 'velocity' of the particles $i$ and $j$ on the light front. Recall that $\vec{k}_{\perp}$ is the two-dimensional momentum and $z$ plays the role of the mass in the Galilean analogy of the light-front dynamics. In these new variables the vertices have very compact form. For example vertex for the transition $+\rightarrow++$

$$
V_{3}\left(3^{+} \rightarrow 1^{+} 2^{+}\right)=2 i g z_{3} v_{21}^{*} .
$$

Coming back to the computation of the gluon wave function, in order to find the expression for this object, one can construct the following recurrence relation valid for the gluon wave function in the $+\rightarrow+\cdots+$ case

$$
\Psi_{n}\left(k_{1}, k_{2}, \ldots, k_{n}\right)=\frac{2 i g}{D_{n}} \sum_{i=2}^{n} \frac{\vec{\varepsilon}_{\perp}^{(+)} \cdot \vec{v}_{i i-1}}{\sqrt{\xi_{i-1 i}}} \Psi_{n-1}\left(k_{1}, \ldots, k_{i-1 i}, \ldots, k_{n}\right)
$$


where $D_{n}$ is the denominator for the last off-shell state with $n$ gluons. This recurrence relation is depicted in Fig. 11.

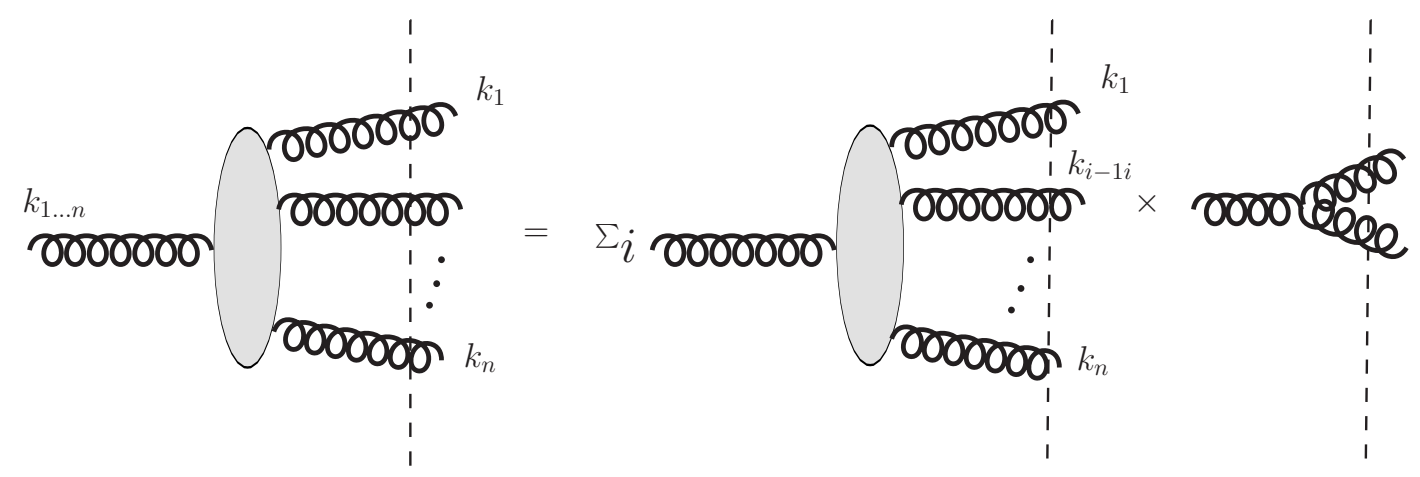

Figure 11: Light-front recurrence relation for the wave function. The sum $\sum_{i}$ is over all the gluons in the final state. Vertical dashed lines indicate the energy denominators.

In the above expression, $\Psi_{n-1}\left(k_{1}, k_{2}, \ldots, k_{i i+1}, \ldots, k_{n}\right)$ (with $\left.k_{i i+1} \equiv k_{i}+k_{i+1}\right)$ is the gluon wave function in the momentum space (with $n-1$ gluons in the final state) before the splitting of the gluon with the momentum $k_{i+1}$, and $\Psi_{n}\left(k_{1}, k_{2}, \ldots, k_{i}, k_{i+1}, \ldots, k_{n}\right)$ is the wave function after splitting of this gluon. The sum goes over all possible splittings of the gluons. In the expression above, the convention has been used where the factors for the intermediate states associated with the longitudinal momentum fractions have been absorbed into the definition of the vertex [38]. This convention results in factors $\sqrt{\xi_{i-1 i}}$ where

$$
\xi_{i j} \equiv \frac{z_{i} z_{j}}{z_{i}+z_{j}}
$$

which depends only on the longitudinal degrees of freedom. It can be interpreted as the reduced mass for a two body problem [35]. We note that all dependence on momenta of the daughter gluons $i$ and $i-1$ is now encoded in two variables: $\xi_{i-1 i}$ and $\vec{v}_{i-1 i}$.

The recurrence relation (152) has been written for the general case where the energy denominator contains the scale $Q^{2}$ which is the scale of the off-shellness of the incoming gluon. In that case however the recurrence formula is rather difficult to solve, though it can be rewritten in an elegant form in the transverse coordinate space with modified Bessel functions [29]. Substantial simplification occurs though when the incoming gluon is taken on-shell, $Q^{2}=0$. It turns out that the recursion can be explicitly solved in this case.

The recurrence formula, i.e. Eq. (152), for the case of the on-shell incoming gluon and the same helicities for the outgoing gluons has the explicit form

$$
\begin{gathered}
-D_{n+1} \Psi_{n+1}(1,2, \ldots, n+1)= \\
=2 i g \frac{v_{12}^{*}}{\sqrt{\xi_{12}}} \Psi_{n}(12,3, \ldots, n+1)+2 i g \frac{v_{23}^{*}}{\sqrt{\xi_{23}}} \Psi_{n}(1,23, \ldots, n+1)+\ldots+2 i g \frac{v_{n n+1}^{*}}{\sqrt{\xi_{n n+1}}} \Psi_{n}(1,2, \ldots, n n+1),
\end{gathered}
$$

with

$$
D_{n+1}=-\left(\frac{\vec{k}_{1 \perp}^{2}}{z_{1}}+\frac{\vec{k}_{2 \perp}^{2}}{z_{2}}+\cdots+\frac{\vec{k}_{n+1 \perp}^{2}}{z_{n+1}}\right)+\frac{\vec{q}_{\perp}^{2}}{z_{0}} .
$$

We have introduced the notation $\Psi_{n}(1, \ldots, i-1 i, \ldots, n+1)$ where $i-1 i$ means that it is the gluon with the transverse momentum $\vec{k}_{i-1 i}=\vec{k}_{i-1}+\vec{k}_{i}$. Again, we recall that we are considering color ordering 
in all these wave functions, therefore color degrees of freedom are absent. The wave function for the incoming state has the normalization $\Psi_{1}(1)=1$. After the first splitting one gets

$$
\Psi_{2}(1,2)=2 i g \frac{1}{\sqrt{\xi_{12}}} \frac{v_{12}^{*}}{\xi_{12} \vec{v}_{12}^{2}}=i g \frac{1}{\sqrt{\xi_{12}}} \frac{1}{\xi_{12} v_{12}} .
$$

According to the recurrence (155), the next splitting leads from $\Psi_{2}(1,2)$ to $\Psi_{3}(1,2,3)$ with the graphs depicted in Fig. 12 and the result is

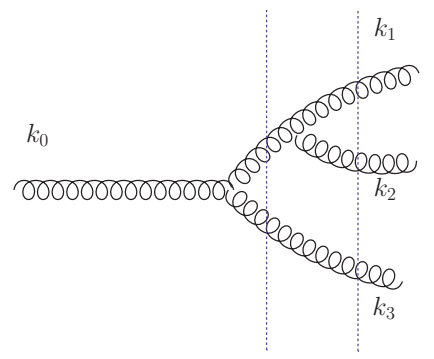

(a)

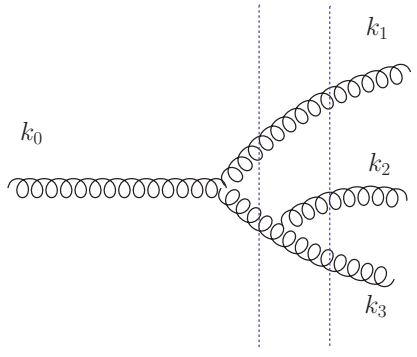

(b)

Figure 12: Light front graphs contributing to the gluon wave function with 3 gluons. Vertical lines indicate the energy denominators.

$$
\begin{aligned}
D_{3} \Psi_{3}(1,2,3) & =2 i g\left[\frac{v_{12}^{*}}{\sqrt{\xi_{12}}} \Psi_{2}(12,3)+\frac{v_{23}^{*}}{\sqrt{\xi_{23}}} \Psi_{2}(1,23)\right] \\
& =-2 g^{2}\left[\frac{v_{12}^{*}}{\sqrt{\xi_{12} \xi_{(12) 3}}} \frac{1}{\xi_{(12) 3} v_{(12) 3}}+\frac{v_{23}^{*}}{\sqrt{\xi_{23} \xi_{1(23)}}} \frac{1}{\xi_{1(23)} v_{1(23)}}\right] \\
& =-2 g^{2} \frac{\sqrt{z_{0}}}{\sqrt{z_{1} z_{2} z_{3}}} \frac{v_{12}^{*} \xi_{1(23)} v_{1(23)}+v_{23}^{*} \xi_{(12) 3} v_{(12) 3}}{\xi_{(12) 3} \xi_{1(23)} v_{(12) 3} v_{1(23)}}
\end{aligned}
$$

where we used $\xi_{12} \xi_{(12) 3}=\xi_{23} \xi_{1(23)}=\frac{z_{1} z_{2} z_{3}}{z_{1}+z_{2}+z_{3}}=\frac{z_{1} z_{2} z_{3}}{z_{0}}$ and $z_{0}=z_{123}$. We can further simplify the numerator of this expression to obtain,

$$
\Psi_{3}(1,2,3)=-g^{2} \frac{\sqrt{z_{0}}}{\sqrt{z_{1} z_{2} z_{3}}} \frac{1}{\xi_{(12) 3} \xi_{1(23)}} \frac{1}{v_{(12) 3} v_{1(23)}} .
$$

Note that, the energy denominator $D_{3}$ disappeared from the equation as it has canceled with the numerator when finding the common denominator for expression (158). This is actually quite an important property and leads to the very simple expression for the resummed gluon wave function. It is also important in recovering some crucial properties of the helicity amplitudes as we shall show later. Comparing the expressions for the $\Psi_{2}$ and $\Psi_{3}$ one can observe the emerging pattern in the structure of these functions. Performing proof by means of the mathematical induction one can demonstrate that when all of the helicities of the outgoing gluons are positive, the wave function can be resummed to the following compact formula [29]

$$
\begin{aligned}
\Psi_{n}(1,2, \ldots, n)= & (i g)^{n-1} \Delta^{(n)} \frac{\sqrt{z_{0}}}{\sqrt{z_{1} z_{2} \ldots z_{n}}} \frac{1}{\xi_{(12 \ldots n-1) n} \xi_{(12 \ldots n-2)(n-1 n) \ldots \xi_{1(2 \ldots n)}}} \\
& \times \frac{1}{v_{(12 \ldots n-1) n} v_{(12 \ldots n-2)(n-1 n)} \ldots v_{1(2 \ldots n)}} .
\end{aligned}
$$


A useful notation has been also introduced above where,

$$
\begin{aligned}
& v_{\left(i_{1} i_{2} \ldots i_{p}\right)\left(j_{1} j_{2} \ldots j_{q}\right)}=\frac{\tilde{k}_{i_{1}}+\tilde{k}_{i_{2}}+\ldots+\tilde{k}_{i_{p}}}{z_{i_{1}}+z_{i_{2}}+\ldots+z_{i_{p}}}-\frac{\tilde{k}_{j_{1}}+\tilde{k}_{j_{2}}+\ldots+\tilde{k}_{j_{q}}}{z_{j_{1}}+z_{j_{2}}+\ldots+z_{j_{q}}}, \\
& \xi_{\left(i_{1} i_{2} \ldots i_{p}\right)\left(j_{1} j_{2} \ldots j_{q}\right)}=\frac{\left(z_{i_{1}}+z_{i_{2}}+\ldots+z_{i_{p}}\right)\left(z_{j_{1}}+z_{j_{2}}+\ldots+z_{j_{q}}\right)}{z_{i_{1}}+z_{i_{2}}+\ldots+z_{i_{p}}+z_{j_{1}}+z_{j_{2}}+\ldots+z_{j_{q}}},
\end{aligned}
$$

together with the complex notation for the transverse vectors

$$
\tilde{k}_{i} \equiv \vec{\varepsilon}_{\perp}^{(-)} \cdot \vec{k}_{i \perp}
$$

We also introduced the partial sums $z_{(1 \ldots i)} \equiv z_{1}+z_{2}+\cdots+z_{i}$ and $\tilde{k}_{(1 \ldots i)} \equiv \tilde{k}_{1}+\tilde{k}_{2}+\cdots+\tilde{k}_{i}$. In formula $(161), \Delta^{(n)}$ is defined to contain the overall momentum conservation $\delta$-functions,

$$
\Delta^{(n)} \equiv \delta^{(2)}\left(\sum_{i=1}^{n} \vec{k}_{i \perp}-\vec{q}_{\perp}\right) \delta\left(1-\sum_{i=1}^{n} z_{i}\right) .
$$

It contains only the longitudinal and transverse components of the momenta. It is a particular feature of the light front formulation, where essentially the light-front energies are not conserved between intermediate states (only on-shell final and initial states satisfy minus component conservation) when they appear in the energy denominators. Since the gluons $1, \ldots, n$ are not on-shell in $\Psi_{n}$ then the light-front energies are not conserved. We shall discuss this issue more later on in the section on the Ward identities. In passing, we shall also note that from the expression (161) we can obtain the helicity amplitude $(-+\cdots+)$ (all gluons on-shell and outgoing) if we multiply by the denominator of the final state (which is equivalent to amputating the propagator) and set the gluons $1,2, \ldots, n$ on-shell. In that case we arrive at the following result

$$
D_{n} \Psi_{n}=0,
$$

since in this case $D_{n}=0$ because it corresponds to the physical on-shell condition (i.e. light-front energies in the initial and final states are then equal) and $\Psi_{n}$ does not contain any singularities at $D_{n}=0$. This result is of course consistent with the vanishing result for the helicity amplitude

$$
\mathcal{M}(-+\cdots+)=0
$$

as expected, see Sec. 3. However, $\Psi_{n}$ in general can be non-zero as it is off-shell. It is interesting that the result about the vanishing of this helicity amplitude stems from the requirement of the energy conservation on the light-front.

\subsection{Relation between spinor helicity formalism and light-front variables}

In Sec. 3 we have discussed the basic ingredients and methods used to compute the amplitudes in QCD using the helicity formalism. We have seen that the helicity amplitudes for many gluons take a very compact form when expressed in terms of the spinor products. In the previous section we have evaluated off-shell gluon wave functions on the light-front and expressed them in variables than naturally appear on the light front, and which correspond to the relative 'velocities' $v_{i j}$ and 'masses' $z_{i}$ as well as 'reduced masses' $\xi_{i j}$. It turns out that those variables are closely related to the spinor products $\langle i j\rangle$.

Comparing expressions for the spinor products, given in Eqs. (85), (86) and Eq. (87) with the lightfront variables $v_{i j}$, Eq. (149) and $\xi_{i j}$ Eq. (153) we see that one can relate both variables in the following way

$$
[j i]=\sqrt{2 z_{i} z_{j}} \varepsilon_{\perp}^{-} \cdot\left(\frac{\vec{k}_{i \perp}}{z_{i}}-\frac{\vec{k}_{j \perp}}{z_{j}}\right)=\sqrt{2 z_{i} z_{j}} v_{i j}, \quad\langle i j\rangle=\sqrt{2 z_{i} z_{j}} \varepsilon_{\perp}^{+} \cdot\left(\frac{\vec{k}_{i \perp}}{z_{i}}-\frac{\vec{k}_{j \perp}}{z_{j}}\right)=\sqrt{2 z_{i} z_{j}} v_{i j}^{*} .
$$


Thus the spinor products are variables that naturally appear in the light-front methods. Note also, that in the framework of the light-front theory the reference momentum $q$ for the polarization vector $\varepsilon_{\mu}(k, q)$ is set equal to the momentum $\eta$. Therefore in the computations on the light-front one uses the same reference momentum for all the gluons. Of course, thanks to the gauge invariance it is equivalent to any choice of the reference momenta, and therefore the results for the on-shell amplitudes are the same on the light-front and using more standard helicity methods.

\subsection{Factorization of the fragmentation functions}

Let us now turn to the analysis of the fragmentation functions within the light-front perturbation theory. We define here the fragmentation function to be an off-shell object, in a particular case when the incoming particle is off-shell and then it fragments to give $n$ particles in the final state. The relevant process is illustrated in Fig. 13. The final state which contains $n$ particles is on-shell in this case. This is converse to the situation with the gluon wave functions where the initial particle is on-shell and the final are off-shell as discussed previously in Sec. 4.1. At the tree level, the diagrams contributing to the wave functions and fragmentation functions are very similar, and the topology is the same. Therefore the results for the same configurations of the helicities are similar, as we shall see later by comparing explicit expressions. Nevertheless, there are some important differences. One important property of the fragmentation functions, that is different from the wave functions, is that of the factorization when the different fragmentation subamplitudes originate from the topologically disconnected graphs.

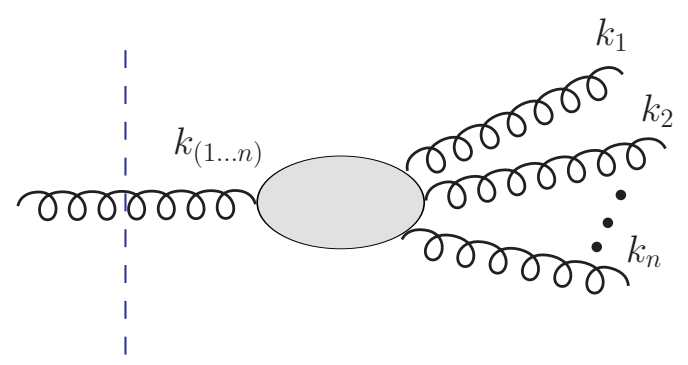

Figure 13: Pictorial representation of the fragmentation amplitude $T[(12 \ldots n) \rightarrow 1,2, \ldots, n]$ for the single off-shell initial gluon. The dashed line denotes the energy denominator which is included in the definition of this object.

Physically, the factorization property of independent tree amplitudes is quite intuitive. One considers subamplitude, such that $m$ parent virtual gluons fragment into $n$ on-shell gluons. To be precise, the first parent gluon, denoted by $\left(12 \ldots n_{1}\right)$ fragments into $n_{1}$ gluons $\left(1,2, \ldots, n_{1}\right)$, the second parent gluon, $\left(n_{1}+1, n_{1}+2, \ldots, n_{2}\right)$ fragments into $n_{2}-n_{1}$ gluons $\left(n_{1}+1, n_{1}+2, \ldots, n_{2}\right)$, and so on. Using the proof by induction, shown in [29], one can then demonstrate that the amplitude of the fragmentation tree $T$ may be factorized into individual fragmentation amplitudes of the parent gluons, $T_{i}$ in the following way,

$$
\begin{aligned}
& T\left[\left(1 \ldots n_{1}\right),\left(n_{1}+1 \ldots n_{2}\right), \ldots,\left(n_{m-1} \ldots n\right) \rightarrow 1,2, \ldots n\right]=T_{1}\left[\left(1 \ldots n_{1}\right) \rightarrow 1,2, \ldots n_{1}\right] \\
\times & T_{2}\left[\left(n_{1}+1 \ldots n_{2}\right) \rightarrow n_{1}+1, n_{1}+2, \ldots n_{2}\right] \times \ldots \times T_{m}\left[\left(n_{m-1}+1 \ldots n\right) \rightarrow n_{m-1}, n_{m-1}+1, \ldots n\right] .
\end{aligned}
$$

The factorization property is rather trivial in the case of standard time-ordered perturbation theory. However, it is not at all obvious in the light-front formulation. This stems from the fact that the different parts of the light-front graphs are correlated by the energy denominators. For a given lightfront time-ordering of the splittings, the variables related to different fragmentation trees are correlated 


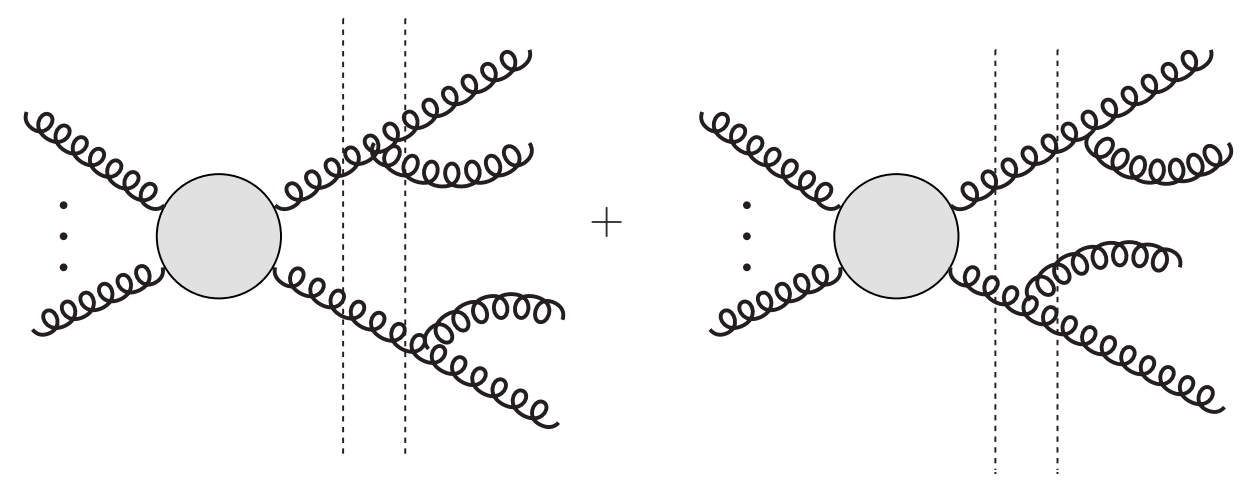

Figure 14: Two sample time ordered diagrams of the same topology but with different time orderings. In each diagram separately there is no factorization as the energy denominators (indicated via dashed vertical lines) couple different parts of the fragmentation trees. The factorization occurs after the both diagrams are added.

in the energy denominators, and the factorization property does not hold for an individual diagram with particular ordering of the vertices on the light-front. The factorization only occurs once the summation over all the time orderings of the vertices in different disconnected parts of the graph is performed. In this case the energy denominators can be written as factorized products of the energy denominators. The full proof by induction is given in Ref. [29], but here we shall give a simple example which illustrates how the procedure works. Consider the diagram with two gluons fragmenting, each into two subsequent gluons which are on-shell. This is illustrated in Fig. 14. The blob denotes the rest of the amplitude which is of no interest to the argument, there could be arbitrary number of gluons which are incoming into the blob. The light-front time flows from left to right and therefore one needs to add both diagrams depicted in Fig. 14 where the splitting of the parent gluons occur in two different time sequences. Let us denote by

$$
A=k_{A, 1}^{-}+k_{A, 2}^{-}-k_{A}^{-},
$$

the contribution to the energy denominator only from the upper graph and by

$$
B=k_{B, 1}^{-}+k_{B, 2}^{-}-k_{B}^{-}
$$

the contribution to the energy denominator from the lower graph. The last two denominators in both diagrams depicted in Fig. 14 will have the following structure

$$
\frac{1}{A+B} \frac{1}{B}+\frac{1}{A} \frac{1}{A+B}=\frac{1}{A B}
$$

Since the vertices are identical in the two graphs we can only consider the energy denominators. This property can be generalized into arbitrary number of splittings in topologically distinct fragmentation trees, leading to the result written in Eq. (169).

The above factorization property can be used then to write down the explicit recursion formula for the fragmentation amplitudes. It is actually a light-front analog of the Berends-Giele recursion relations, which were discussed at length in Sec. 3. It can be written for the arbitrary configurations of the helicity but for now let us restrict here ourselves to the case when the incoming gluon is of helicity + and it fragments into $n+1$ gluons of the same helicity + . In this case, the fragmentation into $n+1$ gluons denoted by $T_{n+1}\left[(1,2, \ldots, n+1)^{+} \rightarrow 1^{+}, 2^{+}, \ldots, n+1^{+}\right]$can be represented by lower fragmentation factors $T_{i}\left[(1 \ldots i)^{+} \rightarrow 1^{+}, \ldots, i^{+}\right]$and $T_{n+1-i}\left[(i+1 \ldots n+1)^{+} \rightarrow i+1^{+}, \ldots, n+1^{+}\right]$and by summing 
over the splitting combinations. To be precise one has

$$
\begin{aligned}
T_{n+1}\left[(12 \ldots n+1)^{+} \rightarrow 1^{+}, 2^{+}, \ldots, n+1^{+}\right]=-\frac{2 i g}{D_{n+1}} \sum_{i=1}^{n}\left\{\frac{v_{(1 \ldots i)(i+1 \ldots n+1)}^{*}}{\sqrt{\xi_{(1 \ldots i)(i+1 \ldots n+1)}}}\right. \\
\left.\times T_{i}\left[(1 \ldots i)^{+} \rightarrow 1^{+}, \ldots, i^{+}\right] T_{n+1-i}\left[(i+1 \ldots n+1)^{+} \rightarrow i+1^{+}, \ldots, n+1^{+}\right]\right\} .
\end{aligned}
$$

Here, the energy denominator $D_{n+1}$ is the denominator for the state with the first incoming off-shell gluon and it is taken as the difference between the light-front energy of this gluon and the sum of the light-front energies of the particles in the final state. This expression is the final state analog of formula (152) for the iteration of the wave function and it is schematically depicted in Fig. 15.

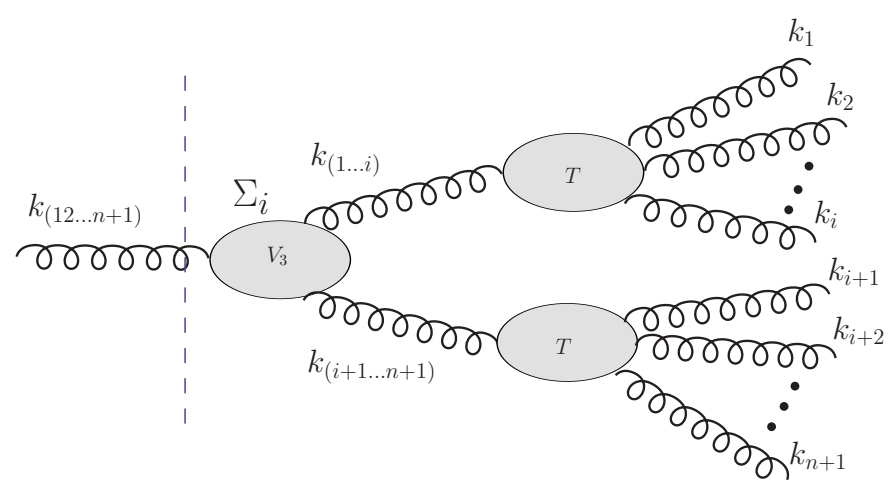

Figure 15: Pictorial representation of the recursion relation for the fragmentation function represented in Eq. (173), using factorization property. This recursion is a light-front analog of the Berends-Giele recursion relation. The helicities of the outgoing gluons are chosen to be the same in this particular case. Dashed vertical line indicates the energy denominator $D_{n+1}$. The blobs denoted by $T$ contain sequence of only triple gluon vertices, due to the particular choice of the helicities for the final state gluon. The summation over the light front time orderings of the splittings in the two blobs $T$, which allows for the factorization, has been already been performed.

We have limited the discussion here to the case of the fragmentation functions at the tree level, however one can show that the factorization holds for the fragmentation functions in the case when the loops are involved as well. For the case with loops it is sufficient to demand that each fragmentation branch of the graph in question is topologically disconnected from the other, within each fragmentation branch however there can be any number of loops inside. The same factorization property holds with any species of particles since the fragmentation property depends only on the light front energy denominators which are universal for all particles (vertices which do depend on the type of the particle are local). In general the factorization does not hold exactly for the wave functions, however it can be shown that it can appear under some approximation also in that case. Such an example is discussed in the next section, where we consider deuteron wave function in the weak binding limit.

\subsection{Cluster decomposition}

The factorization property of the fragmentation functions discussed above, is also related to the cluster decomposition theorem for scattering amplitudes, see Ref. [31, 74, 75] and has been used to derive some interesting properties of the deuteron form factor. We shall recall the basic facts about cluster decomposition for the deuteron following [31]. 
Deuteron is a notoriously difficult object to describe since it is a very complex dynamical system. In the limit of large distances $R \sim 1 / Q$ it can be viewed as weakly bound system of two nucleons. On the other hand at small distances the quark structure has to be taken into account and the deuteron cannot be described using solely nuclear degrees of freedom. Using light-front formalism one can express the deuteron Fock light-front wave function in terms of the different partonic components:

$$
|6 q\rangle,|6 q+g\rangle+\ldots .
$$

Because the binding energy of the deuteron is very small one can assume that the deuteron is dominated by the $|6 q\rangle=|N N\rangle$ component. This light-front wave function was analyzed in Ref. [31] within the model that assumes the following form of the couplings between deuteron and nucleons and nucleons and quarks

$$
\mathcal{L}_{I}=g \phi_{d} \phi_{N} \phi_{N}+h \epsilon_{i j k} \phi_{N} q^{i} q^{j} q^{k},
$$

where $g$ and $h$ represent the coupling constants of the deuteron to two nucleons and a nucleon to three quarks respectively. This model within the light-front formalism, and under the assumption of the zero-binding limit, leads to the factorized expression for the deuteron wave function. Therefore the nuclear wave function in this approximation is the product of the cluster, nucleon wave functions. The relevant light-front diagrams are shown in Fig. 16.
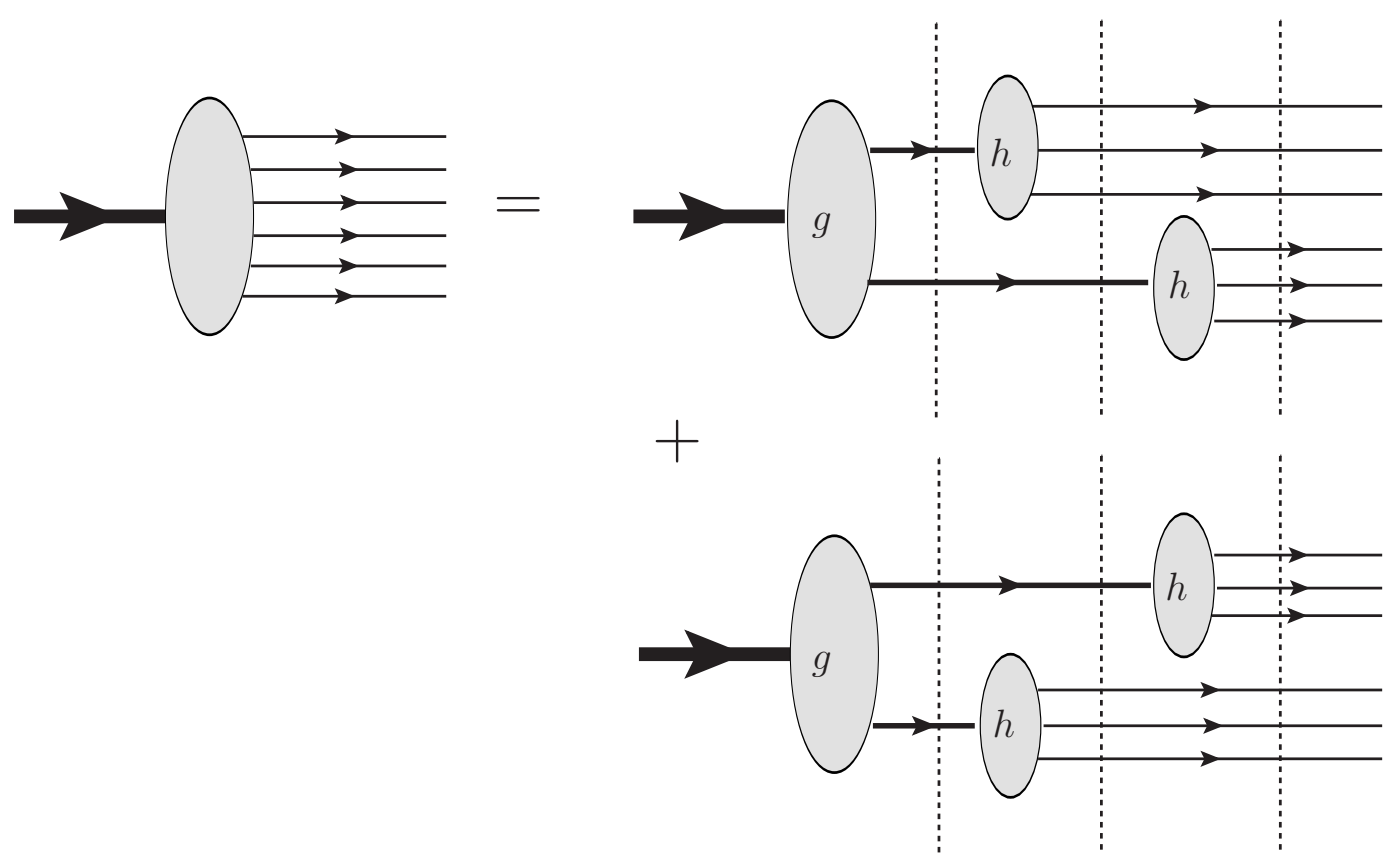

Figure 16: Light-front diagrams which contribute to the relativistic deuteron wave function. The blobs which are labeled $g$ and $h$ denote the effective vertices which are defined in the effective interaction Lagrangian in Eq. (175). The effective interaction provides the clustering of the two nucleons. The sum of the two diagrams factorizes in the weak binding limit.

This factorization in turn leads to various forms of the factorization of the deuteron form factor. If the scale $Q^{2}$ is very small, then this leads to the impulse approximation which however has a rather limited range of applicability. At large values of $Q^{2}$ is can be shown that a different form of factorization arises which involves a reduced form factor.

This example shows how the light-front formalism can be very useful as it leads to the very physical picture of the complex problem such as the structure of the deuteron wave function. In this case the factorization or the cluster decomposition leads naturally to the factorized forms for the deuteron form factor in different kinematical regions. 


\subsection{Fragmentation functions for simple helicity configurations}

Let us compute here the simplest example of the fragmentation function with $+\rightarrow+\cdots+$ helicity. We shall utilize here the recursion relation which uses factorization property discussed before, see Eq. (173). For the simplest helicity configuration $+\rightarrow+\cdots+$ the lowest order fragmentation function has the following expression on the light-front

$$
T_{2}\left[(12)^{+} \rightarrow 1^{+}, 2^{+}\right]=-\frac{2 i g}{D_{2}} \frac{v_{12}^{*}}{\sqrt{\xi_{12}}}=-2 i g \frac{v_{12}^{*}}{\xi_{12}^{3 / 2}\left|\vec{v}_{12}\right|^{2}}=-i g \frac{1}{\xi_{12}^{3 / 2}} \frac{1}{v_{12}},
$$

where

$$
D_{2}=\sum_{i=1}^{2} \frac{\vec{k}_{i \perp}^{2}}{z_{i}}-\frac{\left(\vec{k}_{(12) \perp}\right)^{2}}{z_{(12)}},
$$

for this case. Note that the energy denominator for the initial off-shell gluon is taken here with respect to the final state, which is on-shell.

The factorization formula, Eq. (173) is effectively a recursion formula, which can be solved easily by induction for this choice of helicities. The general result for the tree-level fragmentation function for the splitting of the off-shell gluon with transverse momentum $\vec{k}_{(1 \ldots n), \perp}$ and the longitudinal fraction $z_{(1 \ldots n)}$ reads $[29]$

$$
T_{n}\left[(12 \ldots n)^{+} \rightarrow 1^{+}, 2^{+}, \ldots, n^{+}\right]=(-i g)^{n-1}\left(\frac{z_{(12 \ldots n)}}{z_{1} z_{2} \ldots z_{n}}\right)^{3 / 2} \frac{1}{v_{12} v_{23} \ldots v_{n-1 n}} .
$$

The off-shell gluon is labeled by $(12 \ldots n)$ and the final state $n$ on-shell gluons are labeled as $1,2, \ldots n$. These $n$ final state gluons have transverse momenta $\vec{k}_{1 \perp}, \ldots, \vec{k}_{n \perp}$ and the longitudinal momentum fractions $z_{1}, \ldots, z_{n}$. The fragmentation function is depicted in Fig. 13.

In a similar manner one can evaluate other fragmentation functions for the different helicity configurations. The other rather simple example is that of $(-\rightarrow-+\cdots+)$. For the off-shell incoming gluon the result is non-zero and reads

$$
T_{n}\left[(12 \ldots n)^{-} \rightarrow 1^{-}, 2^{+}, \ldots, n^{+}\right]=(i g)^{n-1}\left(\frac{z_{1}}{z_{1 \ldots n}}\right)^{2}\left(\frac{z_{1 \ldots n}}{z_{1} \ldots z_{n}}\right)^{3 / 2} \frac{1}{v_{n n-1} v_{n-1 n-2} \ldots v_{21}} .
$$

In fact up to the normalization factor $\left(z_{1} / z_{1 \ldots n}\right)^{2}$ this amplitude is exactly equivalent to the $(+\rightarrow$ $+\cdots+$ ) given above in Eq. (178). This is to be expected as they both correspond to the same (offshell) helicity amplitudes.

Note that, if we change (in any of these fragmentation functions) the incoming gluon into the outgoing one, then this would correspond to the off-shell amplitude with $(+-\cdots+)$ configuration. To obtain the on-shell amplitude one needs to amputate the propagator and set the incoming momentum on-shell which is equivalent to

$$
\left.\left(D_{n+1} T_{n}\left[(12 \ldots n)^{-} \rightarrow 1^{-}, 2^{+}, \ldots, n^{+}\right]\right)\right|_{D_{n+1}=0}=0
$$

as the function $T_{n}$ does not have any singularities at $D_{n+1}$. This is of course consistent with the fact that the amplitude $\mathcal{M}(-+\cdots+)$ vanishes in the on-shell case. This is the same property as discussed before for the wave function. The form of the fragmentation function is very similar to the form of the wave function for the same combination of helicities, except that the way the momenta are clustered in the $v$ variables is slightly different. In the case of the fragmentation functions the $v_{i-1 i}$ variables depend on the momenta of the adjacent gluons $i$ and $i-1$. On the other hand, in the case of the wave function, the 
dependence on the transverse momenta of gluons is factorized into reciprocals of $v_{(12 \ldots p)(p \ldots n)}$ with the index $p$ taking all possible positions from $p=2$ to $p=n-1$. One can show however that, interestingly, the fragmentation and wave functions can be recast into each other by using the Fourier transform from the momentum to transverse coordinate space. The wave function given by (161) may be also expressed in the following way:

$$
\Psi_{n}(1,2, \ldots, n)=(i g)^{n-1} \frac{1}{\sqrt{z_{1} z_{2} \ldots z_{n}}} \frac{1}{\tilde{k}_{(1)} \tilde{k}_{(12)} \ldots \tilde{k}_{(12 \ldots n-1)}} .
$$

where we have used the identity,

$$
v_{(1 \ldots i)(i+1 \ldots n)} \xi_{(1 \ldots i)(i+1 \ldots n)}=\left(\frac{\tilde{k}_{1}+\ldots+\tilde{k}_{i}}{z_{1}+\ldots+z_{i}}+\frac{\tilde{k}_{1}+\ldots \tilde{k}_{i}}{1-\left(z_{1}+\ldots+z_{i}\right)}\right) \xi_{(1 \ldots i)(i+1 \ldots n)}=\sum_{j=1}^{i} \tilde{k}_{j}
$$

and introduced the notation $\tilde{k}_{(1 \ldots i)} \equiv \tilde{k}_{1}+\ldots+\tilde{k}_{i}$. Recall that complex vectors $\tilde{k}_{i}$ here are constructed from transverse vectors $\vec{k}_{i \perp}$, see Eq. (164). One may go back to the real representation of the transverse vectors and perform the Fourier transform of (181),

$$
\Phi_{n}\left(z_{1}, \vec{r}_{1} ; \ldots ; z_{n}, \vec{r}_{n}\right)=\int \frac{d^{2} \vec{k}_{1 \perp}}{(2 \pi)^{2}} \ldots \frac{d^{2} \vec{k}_{n \perp}}{(2 \pi)^{2}} \exp \left(i \vec{k}_{1 \perp} \cdot \vec{r}_{1}+\ldots+i \vec{k}_{n \perp} \cdot \vec{r}_{n}\right) \Psi_{n}\left(z_{1}, \vec{k}_{1 \perp}, \ldots, z_{n}, \vec{k}_{n \perp}\right)
$$

where $\Phi_{n}$ is the wave function in the coordinate space. It is convenient to change the momentum variables:

$$
\left\{\vec{k}_{1 \perp}, \vec{k}_{2 \perp}, \ldots \vec{k}_{n \perp}\right\} \rightarrow\left\{\vec{k}_{(1) \perp}, \vec{k}_{(12) \perp}, \ldots, \vec{k}_{(12 \ldots n) \perp}\right\}
$$

The Jacobian of the transformation is trivial, and one expresses the gluon momenta in the following way:

$$
\vec{k}_{1 \perp}=\vec{k}_{(1) \perp}, \quad \vec{k}_{2 \perp}=\vec{k}_{(12) \perp}-\vec{k}_{(1) \perp}, \quad \ldots, \quad \vec{k}_{n \perp}=\vec{k}_{(12 \ldots n) \perp}-\vec{k}_{(12 \ldots n-1) \perp} .
$$

The Fourier exponent takes the form,

$$
\begin{aligned}
\exp \left(i \vec{k}_{1 \perp}\right. & \left.\cdot \vec{r}_{1}+\ldots+i \vec{k}_{n \perp} \cdot \vec{r}_{n}\right)= \\
& =\exp \left(i \vec{k}_{(1) \perp} \cdot\left(\vec{r}_{1}-\vec{r}_{2}\right)+i \vec{k}_{(12) \perp} \cdot\left(\vec{r}_{2}-\vec{r}_{3}\right)+\ldots i \vec{k}_{(12 \ldots n-1) \perp} \cdot\left(\vec{r}_{n-1}-\vec{r}_{n}\right)+i \vec{k}_{(12 \ldots n) \perp} \cdot \vec{r}_{n}\right)
\end{aligned}
$$

and the final answer for the wave function in position space reads

$$
\Phi_{n}\left(z_{1}, \vec{r}_{1} ; \ldots ; z_{n}, \vec{r}_{n}\right)=(i g)^{n-1} \delta\left(1-\sum_{i=1}^{n} z_{i}\right) \frac{1}{\sqrt{z_{1} z_{2} \ldots z_{n}}} \frac{1}{\vec{\varepsilon}_{\perp}^{(-)} \cdot \vec{r}_{12} \vec{\varepsilon}_{\perp}^{(-)} \cdot \vec{r}_{23} \ldots \vec{\varepsilon}_{\perp}^{(-)} \cdot \vec{r}_{n-1 n}} .
$$

Note that this form of the wave function in the transverse coordinate space is almost identical (up to the $z$ factors) to the fragmentation function when the change of variables is performed, i.e. coordinate vectors $r_{i j}$ are used in the wave function in place of $v_{i j}$ variables in the fragmentation functions.

\section{Scattering amplitudes in the light-front formalism}

Having discussed the wave functions and fragmentation functions one can now proceed to the analysis of the scattering amplitudes on the light-front. The off-shell color ordered amplitudes for the helicities $(-+\cdots+)$ (in the convention of all outgoing momenta) within the light-front theory have been derived in $[30,32,76]$. One starts with the observation that in order to obtain the $2 \rightarrow n$ amplitudes it is actually easier to consider $1 \rightarrow n$ transitions, as they involve substantially smaller number of light front 
graphs. These $1 \rightarrow n$ transitions, with the incoming gluon off-shell and outgoing on-shell, are actually equivalent to the fragmentation functions discussed previously. One can then construct the recursion relations between the different fragmentation functions and solve them by means of the mathematical induction in order to obtain the off-shell amplitude. In [32] it was shown that this solution can then be expressed as another recursion relation, but this time involving different amplitudes, some of which have MHV-like form even though they are off-shell. The origin of this new recursion formula was then understood as the direct consequence of the gauge invariance property on the light front. Namely, the off-shell object computed from the light-front diagrams is not gauge invariant, but in the recursion relation a new object appears which actually is gauge invariant. It can then be shown that is satisfies the Ward identities with respect to the on-shell legs [33]. Remarkably this new recursion relation is identical to the one, which was found using the matrix elements of straight Wilson lines [34]. In this section we shall discuss these developments using the notation and convention from [32, 33] where more details can be found in these references.

\subsection{From fragmentation functions to $2 \rightarrow n$ amplitudes}

The objective here is to compute all the (tree-level) $2 \rightarrow n$ helicity amplitudes, which were derived previously using standard methods of perturbative QCD, but this time utilizing the framework of the light-front perturbation theory. Since the light front computations simplify for the helicity amplitudes and spinor products emerge naturally as the variables of the non-relativistic kinematics on the lightfront, this alternative derivation can possibly give a new insight into the structure of the quantum theory. In [30] it has been demonstrated that the most efficient way to perform this task is to consider $1 \rightarrow n$ transitions, either via wave functions or fragmentation functions, and then by inverting the momentum flow of one of the gluons, and setting all the particles on-shell, the $2 \rightarrow n$ amplitude is automatically obtained. Such crossing relations are quite intuitive, and obvious in the case of the standard perturbation theory, as the graphs in two different cases are topologically equivalent and one can preform analytical continuation of the amplitude to a different domain of momenta. In the case of the LFPT however, it is not an entirely obvious relation. This stems from the fact that the energy denominators, which entangle the momenta of the particles in a given intermediate state, in principle change via such operation and, moreover, some graphs are vanishing.

One can nevertheless prove, that such relation must be true on the light-front, by starting from the analysis of low order graphs and generalizing the procedure to an arbitrary number of the external particles.

Let us first consider the case of 2 to 3 gluon scattering to illustrate the specifics of the problem and the arguments leading to the crossing relation on the light-front. We want to find the diagrams contributing to the amplitude $2 \rightarrow 3$ from the diagrams which are needed to evaluate the gluon wave function for the 1 to 4 gluon transition in the $(+\rightarrow++++)$ configuration. The particular helicity configuration is not important here, it has been chosen solely for the purpose of the simplification as four-gluon vertices and Coulomb exchanges will not contribute. As is known from the previous sections the on-shell amplitude for this helicity configuration vanishes, whereas its off-shell counterpart in principle does not (and is not gauge invariant). The $1 \rightarrow 4$ gluon wave function contains $(4-1) !=6$ graphs, for this choice of the helicity.

The way to obtain diagrams for $2 \rightarrow 3$ scattering is by taking the gluon wave function and changing the one gluon line from outgoing into incoming. This procedure is illustrated in Fig. 17, on the left graph, in this case the line that it reversed corresponds to the momentum $k_{1}$. Now the problem on the light-front is that since the diagrams are ordered in light-front time then naively, one recovers only one diagram from the wave functions, which is also shown in this figure, on the right hand side graph. However, since our calculation is being done in the light-front perturbation theory, we need to find all possible time-orderings. To be precise, from the one diagram depicted on the left hand side of Fig. 17 we 

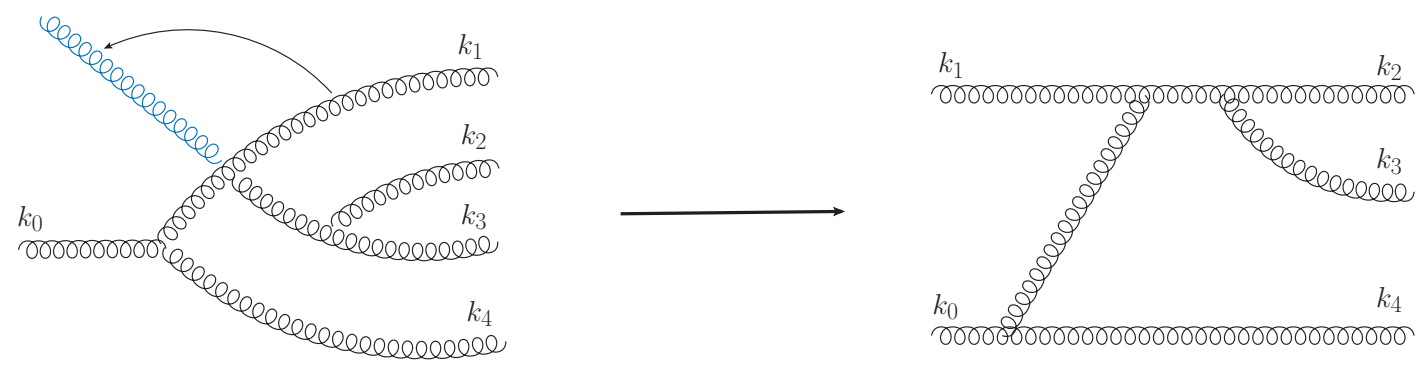

Figure 17: Left: the example of the light-front diagram contributing to the wave function $1 \rightarrow 4$. Right: the diagram which contributes to $2 \rightarrow 3$ amplitude. This diagrams is obtained from the former by changing the momentum $k_{1}$ from outgoing to incoming.

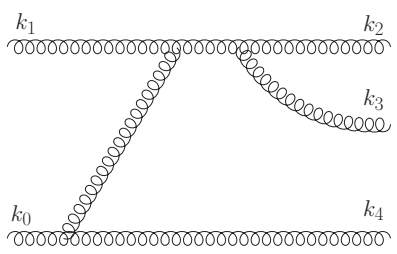

(a) Subgroup $G_{1,1}$
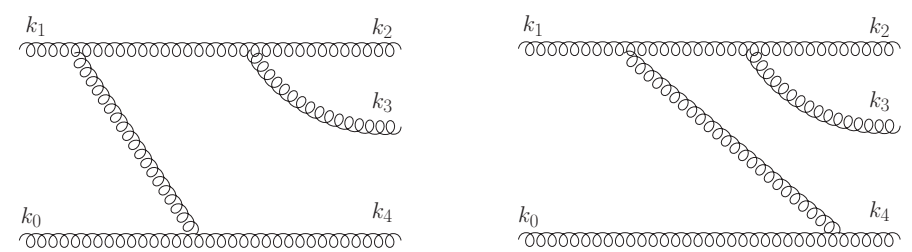

(b) Subgroup $G_{1,2}$

Figure 18: A group of topologically equivalent diagrams which contribute to the $2 \rightarrow 3$ amplitude. These diagrams are obtained from the continuation of the $k_{1}$ momentum, as shown in Fig. 17.

need to obtain three diagrams for $2 \rightarrow 3$ transition of the same topology, which are depicted in Fig. 18 . This continuation and summation needs to the performed with all the diagrams in the $\Psi_{4}$ wave function to arrive at all the corresponding diagrams needed to calculate $\mathcal{M}_{2 \rightarrow 3}$, which are depicted in Figs. 18-21. All the vacuum diagrams have been already excluded here since they vanish on the light-front.

In Figs. 18-21 all the graphs are shown that need to be computed for the amplitude $\mathcal{M}_{2 \rightarrow 3}$. They are organized such that all the graphs with the same topology are grouped together. These groups are in turn composed of smaller subgroups where the directions of the intermediate lines are taken into account. Recall that, on the light front all the lines must have a positive longitudinal momentum. Thus, different kinematical conditions which are satisfied by the longitudinal momenta of the external lines apply for the different subgroups inside a group, in order to satisfy the positivity condition for the internal lines. To make this statement more transparent, we can introduce the following classification for the graphs. Let us call $G_{i}$ the group of diagrams which have the same topology. For example group $G_{2}$ is the group depicted in Fig. 19 and another distinct group, $G_{3}$, in Fig. 20. We denote by $G_{i, j}$ the subgroup of diagrams in group $G_{i}$ which are distinguished by the different kinematical conditions on the external momenta. In our example $G_{2, j}$ denote the subgroups listed in plots a-c in Fig. 19.

Let

$$
\begin{gathered}
s_{2, j} \equiv \text { sum of graphs in subgroup } G_{2, j}, \\
S_{2} \equiv \text { sum of graphs in group } G_{2} .
\end{gathered}
$$

We can then write

$$
S_{2}=s_{2,1} \Theta\left(z_{0}-z_{4}\right) \Theta\left(z_{2}-z_{1}\right)+s_{2,2} \Theta\left(z_{4}-z_{0}\right) \Theta\left(z_{1}-z_{2}\right)+s_{2,3} \Theta\left(z_{0}-z_{4}\right) \Theta\left(z_{1}-z_{2}\right) .
$$

This decomposition arises because all the internal lines must have positive longitudinal momenta. However, it can be easily demonstrated [30] that contributions from all the graphs are actually the same, i.e. $s_{2} \equiv s_{2,1}=s_{2,2}=s_{2,3}$. Therefore,

$$
S_{2}=s_{2}
$$




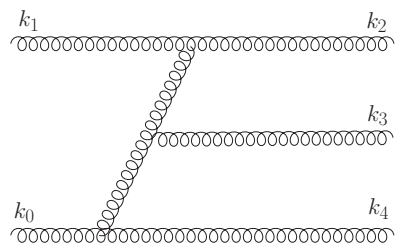

(a) Subgroup $G_{2,1}$

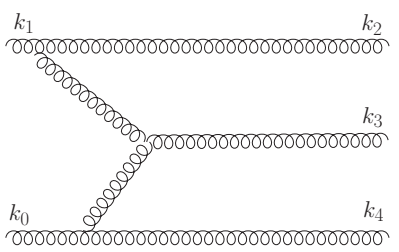

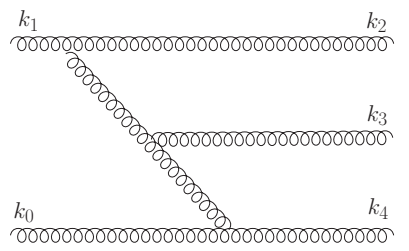

(b) Subgroup $G_{2,2}$

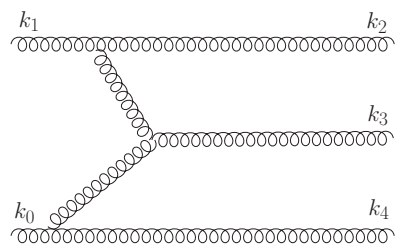

(c) Subgroup $G_{2,3}$

Figure 19: A group of topologically equivalent diagrams for $2 \rightarrow 3$ amplitude with different timeorderings. See text for the explanation
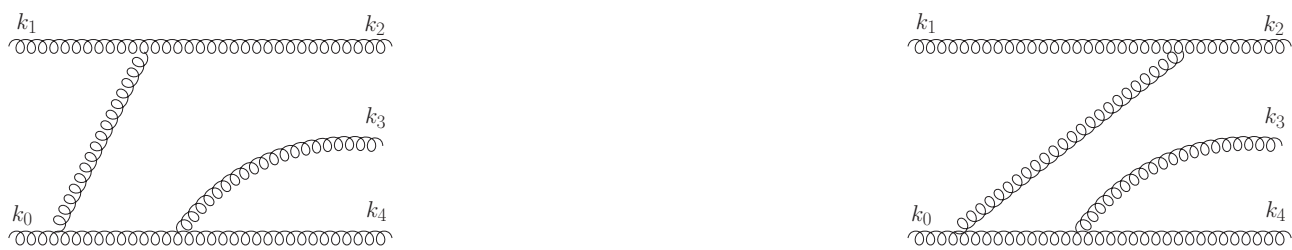

(a) Subgroup $G_{3,1}$

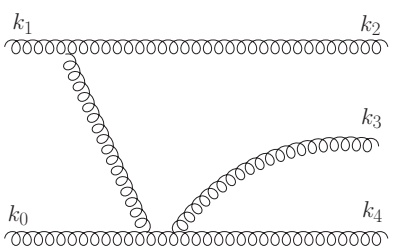

(b) Subgroup $G_{3,2}$

Figure 20: A group of topologically equivalent diagrams for $2 \rightarrow 3$ amplitude with different timeorderings. See text for the explanation.
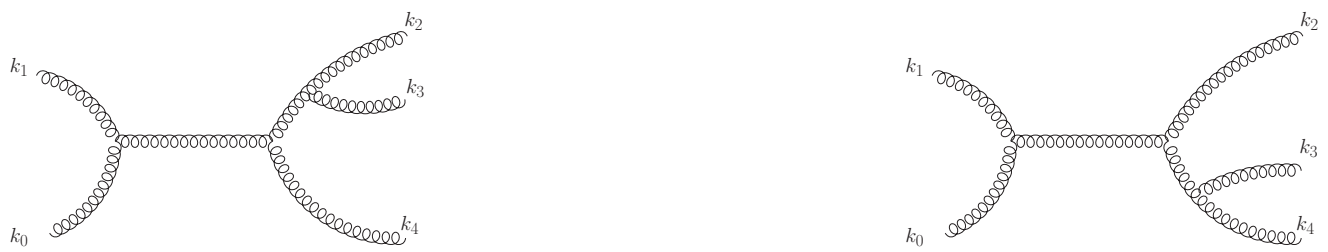

Figure 21: $s$ - channel graph contributions to $2 \rightarrow 3$ scattering. Groups $G_{5}$ and $G_{6}$ are shown on the left and right respectively. Both consist of only one graph and are topologically distinct. 


\begin{tabular}{cc}
2 to $n$ & 1 to $n+1$ \\
\hline$\vec{k}_{0 \perp}+\vec{k}_{1 \perp}=\sum_{i=2}^{n+1} \vec{k}_{i \perp}$ & $\vec{k}_{0 \perp}=\sum_{i=1}^{n+1} \vec{k}_{i \perp}$ \\
$z_{0}+z_{1}=\sum_{i=2}^{n+1} z_{i}$ & $z_{0}=\sum_{i=1}^{n+1} z_{i}$ \\
$\frac{\vec{k}_{0 \perp}^{2}}{z_{0}}+\frac{\vec{k}_{1 \perp}^{2}}{z_{1}}=\sum_{i=2}^{n+1} \frac{\vec{k}_{i \perp}^{2}}{z_{i}}$ & $\frac{\vec{k}_{0 \perp}^{2}}{z_{0}}=\sum_{i=1}^{n+1} \frac{\vec{k}_{i \perp}^{2}}{z_{i}}$ \\
\hline
\end{tabular}

Table 3: Physical conditions for gluon scattering for the case of the $2 \rightarrow n$ and $1 \rightarrow n+1$ amplitudes.

This sum contains all possible physical arrangements of the kinematical conditions on the external lines, so one can remove the $\Theta$ functions from Eq. (189).

As is evident from the figures, some groups consist of several subgroups but there are also some groups which consist of only a single subgroup. Typically these diagrams will be $s$-channel and the internal lines are guaranteed to have a positive longitudinal momentum regardless of the momenta in the external lines. An example of these $s$-channel diagrams is shown in Fig. 21. For arbitrary number of external legs, in every group (including the ones with a single subgroup), all of the $m$ diagrams in one of the subgroups can be obtained from $m$ distinctive diagrams belonging to the wave function $\Psi_{n}$. Furthermore, if the diagrams in one group can be obtained from a certain set of diagrams belonging to $\Psi_{n}$, then the diagrams in another group have to be obtained from a completely different set of diagrams, since the groups are topologically distinct. According to the argument given in Eq. (189) the value of the diagrams for subgroups inside the group are the same, therefore in order to calculate $\mathcal{M}_{2 \rightarrow n}$ all we need are the $n$ ! graphs that can be obtained by simply changing the direction of the $k_{1}$ line in each of the $n$ ! graphs in the 1 to $n+1$ gluon wave function $\Psi_{n+1}$. We demonstrate this step for an arbitrary number of gluons below. One can show that the expression for the light-front diagram $G$ for the $2 \rightarrow n$ is equal to the negative value of the expression of the $1 \rightarrow n+1$ diagram $H$ from which $G$ was obtained. In general we know, see for example Ref. [36], that a light-front graph will be equal to

$$
\frac{\mathcal{V}}{\mathcal{Z D}}
$$

with

$$
\begin{gathered}
\mathcal{V} \equiv \prod_{\text {all vertices }} v_{k}, \\
\mathcal{Z} \equiv \prod_{\text {internal lines }} z_{i}, \\
\mathcal{D} \equiv \prod_{\text {intermediate states }} D_{i},
\end{gathered}
$$

Thus, we need to compare $\mathcal{Z}, \mathcal{V}$ and $\mathcal{D}$ for diagrams $G$ and $H$.

It is important to set up the physical conditions for each of the cases, that is for the $2 \rightarrow n$ and $1 \rightarrow n$ transitions. These conditions are listed in Table 3 . We note that the on-shell condition for the initial and final states has been included. Therefore the light-front energies (or minus components) are equal for these states.

In order to compare the two expressions it is convenient to introduce two auxiliary variables $\vec{k}_{A}$ and $z_{A}$. These will be defined differently for the two cases: $1 \rightarrow n+1$ and $2 \rightarrow n$. For the $2 \rightarrow n$ transition $\vec{k}_{A \perp} \equiv-\vec{k}_{1 \perp}, z_{A} \equiv-z_{1}$ whereas for $1 \rightarrow n+1$ case $\vec{k}_{A \perp} \equiv \vec{k}_{1 \perp}, z_{A} \equiv z_{1}$. It can be seen that, these definitions allow to use the same mathematical expression for both cases. To be precise, for both $2 \rightarrow n$ 
and $1 \rightarrow n+1$ the physical conditions are

$$
\begin{gathered}
\vec{k}_{0 \perp}=\vec{k}_{A \perp}+\sum_{i=2}^{n+1} \vec{k}_{i \perp}, \\
z_{0}=z_{A}+\sum_{i=2}^{n+1} z_{i}, \\
\frac{\vec{k}_{0 \perp}^{2}}{z_{0}}=\frac{\vec{k}_{A \perp}^{2}}{z_{A}}+\sum_{i=2}^{n+1} \frac{\vec{k}_{i \perp}^{2}}{z_{i}} .
\end{gathered}
$$

Let us take as an example the graphs in Fig. 17 and compare the $\mathcal{Z}$ factors. Looking at these it is clear that, using $k_{A}$ and $z_{A}$, we can write,

$$
\begin{aligned}
& \vec{k}_{l \perp}=\vec{k}_{0 \perp}-\vec{k}_{4 \perp}=\vec{k}_{A \perp}+\vec{k}_{2 \perp}+\vec{k}_{i \perp}, \quad z_{l}=z_{0}-z_{4}=z_{A}+z_{2}+z_{3}, \\
& \vec{k}_{q \perp}=\vec{k}_{0 \perp}-\vec{k}_{4 \perp}-\vec{k}_{A \perp}=\vec{k}_{2 \perp}+\vec{k}_{3 \perp}, \quad z_{q}=z_{0}-z_{4}-z_{A}=z_{2}+z_{3},
\end{aligned}
$$

where $q, l$ subscripts denote two internal lines in graphs in Fig. 17. Therefore the $\mathcal{Z}$ does not change from graphs $2 \rightarrow n$ and $1 \rightarrow n+1$.

Next, let us inspect the vertex factors $\mathcal{V}$. Changing the direction of the $k_{1}$ line only one vertex is changed which is the one with the $k_{1}$ line $\left(v_{1}\right)$. The other vertices remain the same and, because the expressions for the internal lines do not change, neither do the expression for these vertices. However, the expression for $v_{1}$ does change: it has an extra minus sign, i.e., $v_{1,2 \rightarrow n}=-v_{1,1 \rightarrow n+1}$. Therefore the vertex factor contains an extra sign, which originates from this vertex, i.e. $\mathcal{V}_{2 \rightarrow n}=-\mathcal{V}_{1 \rightarrow n+1}$. Finally, let us consider the energy denominators $\mathcal{D}$ in a general $2 \rightarrow 2$ graph with arbitrary number of vertices and internal lines. Here, we are mostly considering tree level amplitudes but the arguments are true beyond that. Therefore the diagram can also contain loops, and it is schematically depicted in Fig. 22. Let $Q$ denote the point where gluon labeled as 1 is attached to the rest of a graph. Using it as a reference, we split the graph into a left subgraph and a right subgraph. Let us denote by $D_{a}$ and $D_{b}$ the energy denominators of the intermediate states $a$ and $b$ which are present just next to the vertex where the gluon attaches, in doing that we thus assume that there are more vertices to the left and right of the vertex $Q$, such that $a$ and $b$ are the intermediate states. For Fig. 22a we can write

$$
\begin{aligned}
& D_{a}=\frac{\vec{k}_{0 \perp}^{2}}{z_{0}}-E_{a}^{-}=\sum_{i=2}^{n} \frac{\vec{k}_{i \perp}^{2}}{z_{i}}-E_{a}^{-}-\frac{\vec{k}_{1 \perp}^{2}}{z_{1}}=\frac{\vec{k}_{A \perp}^{2}}{z_{A}}+\sum_{i=2}^{n} \frac{\vec{k}_{i \perp}^{2}}{z_{i}}-E_{a}^{-}, \\
& D_{b}=\frac{\vec{k}_{0 \perp}^{2}}{z_{0}}+\frac{\vec{k}_{1 \perp}^{2}}{z_{1}}-E_{b}^{-}=\frac{\vec{k}_{0 \perp}^{2}}{z_{0}}-\frac{\vec{k}_{A \perp}^{2}}{z_{A}}-E_{b}=\sum_{i=2}^{n} \frac{\vec{k}_{i \perp}^{2}}{z_{i}}-E_{b}^{-},
\end{aligned}
$$

where $E_{a}^{-}$and $E_{b}^{-}$are the sums of the light-front energies in intermediate states $a$ and $b$ respectively, with the assumption that the energy of gluon 1 is excluded from the sum $E_{a}^{-}$. We also used the conservation of the minus components for the initial and final states. Similarly, for Fig. 22b we can write

$$
\begin{aligned}
& D_{a}=\frac{\vec{k}_{0, \perp}^{2}}{z_{0}}-E_{a}^{-}=\frac{\vec{k}_{1, \perp}^{2}}{z_{1}}+\sum_{i=2}^{n} \frac{\vec{k}_{i, \perp}^{2}}{z_{i}}-E_{a}^{-}=\frac{\vec{k}_{A, \perp}^{2}}{z_{A}}+\sum_{i=2}^{n} \frac{\vec{k}_{i, \perp}^{2}}{z_{i}}-E_{a}^{-}, \\
& D_{b}=\frac{\vec{k}_{0, \perp}^{2}}{z_{0}}-\frac{\vec{k}_{1, \perp}^{2}}{z_{1}}-E_{b}^{-}=\frac{\vec{k}_{0, \perp}^{2}}{z_{0}}-\frac{\vec{k}_{A, \perp}^{2}}{z_{A}}-E_{b}^{-}=\sum_{i=2}^{n} \frac{\vec{k}_{i, \perp}^{2}}{z_{i}}-E_{b}^{-} .
\end{aligned}
$$


This time the light-front energy of the gluon 1 has been excluded from $E_{b}^{-}$. Comparing the denominators $D_{a}$ and $D_{b}$ for both cases once again one sees that, using $\vec{k}_{A}$ and $z_{A}$, the expressions are the same, though in each of these cases $\vec{k}_{A}$ and $z_{A}$ are defined differently. Hence, the contribution from the energy denominators $\mathcal{D}$ also does not change. This result is true for all the graphs in the light-front theory with arbitrary topology. One can therefore conclude finally that the $1 \rightarrow n+1$ and $2 \rightarrow n$ scattering amplitudes are equivalent up to a phase and satisfy the following crossing relation

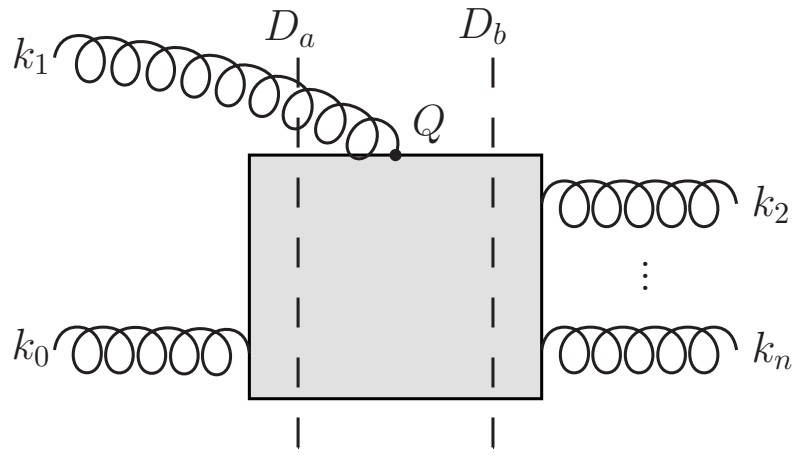

(a)

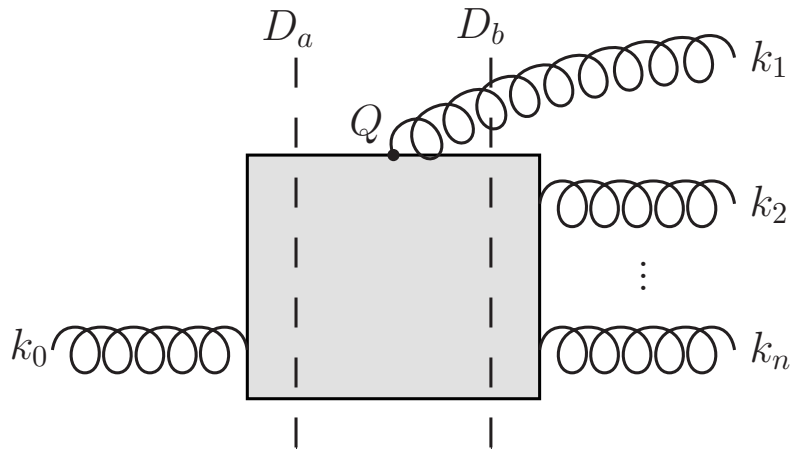

(b)

Figure 22: Schematic representation of the crossing of the momentum of gluon 1 to relate the graphs for $1 \rightarrow n+1$ with graphs $2 \rightarrow n$. Point $\mathrm{Q}$ denotes the vertex at which gluon 1 attaches to the rest of the diagram. $D_{a}$ and $D_{b}$ denote the energy denominators for the intermediate states.

$$
\begin{aligned}
\mathcal{M}_{2 \rightarrow n}\left(\left\{\vec{k}_{0 \perp}, z_{0} ; \vec{k}_{1 \perp}, z_{1}\right\} ;\right. & \left.\left\{\vec{k}_{2 \perp}, z_{2} ; \ldots ; \vec{k}_{n, \perp}, z_{n}\right\}\right)= \\
& -\left.\mathcal{M}_{1 \rightarrow n+1}\left(\left\{\vec{k}_{0 \perp}, z_{0}\right\} ;\left\{\vec{k}_{A \perp}, z_{A} ; \vec{k}_{2 \perp}, z_{2} ; \ldots ; \vec{k}_{n, \perp}, z_{n}\right\}\right)\right|_{\vec{k}_{A \perp} \rightarrow-\vec{k}_{1 \perp}, z_{A} \rightarrow-z_{1}}
\end{aligned}
$$

Furthermore, since the physical conditions for both cases are the same when written in terms of $\vec{k}_{A}$ and $z_{A}$, we can use them to simplify $\mathcal{M}_{2 \rightarrow n}$ by simplifying $\mathcal{M}_{1 \rightarrow n+1}$ first as much as possible.

The relation to off-shell $2 \rightarrow n$ amplitudes is valid both for the case of the wave functions and for the case of the fragmentation functions. In practice, fragmentation functions were used to compute the off-shell amplitudes as they obey the factorization property which then can be used to construct useful recursion relations.

It turns out, that in order to find the on-shell amplitudes $\mathcal{M}_{2 \rightarrow n}$ or equivalently $\mathcal{M}_{1 \rightarrow n+1}$ an off-shell amplitude needs to be used. We shall denote this amplitude by

$$
\underline{\mathcal{M}}_{1 \rightarrow n+1} \text {. }
$$

This object is obtained by assuming that the incoming gluon is off-shell. Thus the off-shell amplitude $\underline{\mathcal{M}}_{1 \rightarrow n+1}$ is more general than $\mathcal{M}_{1 \rightarrow n+1}$ in the sense that

$$
\mathcal{M}_{1 \rightarrow n+1}=\left.\underline{\mathcal{M}}_{1 \rightarrow n+1}\right|_{D_{n+1} \rightarrow 0},
$$

where $D_{n+1}$ is the energy denominator for the first state, i.e. evaluated as the difference between the energies of the final (on-shell) state and the first (initial) off-shell state. In [76], one particular configuration of helicities was considered, $\underline{\mathcal{M}}_{1 \rightarrow n+1}(+\rightarrow-+\cdots+)$, which corresponds to transition 
of $2 \rightarrow n$ particles for $(++\rightarrow+\cdots+)$ when the two particles are incoming and the rest is outgoing or to scattering amplitude $(--+\cdots+)$ when all the particles are outgoing. For the on-shell case this is of course the MHV amplitude.

The off-shell amplitude $\underline{\mathcal{M}}$ has truncated propagator, i.e. the energy denominator has been factored out. Since its incoming gluon is off-shell and all the outgoing ones are on-shell it is directly proportional to the fragmentation function $T_{n}$ discussed before in Sec. 4.3.

The difference between these two objects (apart from some trivial normalization factors) is the denominator for the off-shell initial state which is included inside the fragmentation function. This relationship between $\underline{\mathcal{M}}_{1 \rightarrow n}$ and $T_{n}\left[(12 \ldots n)^{+} \rightarrow 1^{-}, 2^{+}, \ldots, n^{+}\right]$can be expressed as

$$
T_{n}\left[(12 \ldots n)^{+} \rightarrow 1^{-}, 2^{+}, \ldots, n^{+}\right]=\frac{1}{\sqrt{z_{1 \ldots n} z_{1} \ldots z_{n}}} \frac{i}{D_{n}} \underline{\mathcal{M}}_{1 \rightarrow n}\left((12 \ldots n)^{+} \rightarrow 1^{-}, 2^{+}, \ldots, n^{+}\right) .
$$

The normalization factors $1 / \sqrt{\cdots}$ stem from the fact that the fragmentation functions do include $1 / \sqrt{z}$ factor for each of the legs in the vertices that it contains. This is convention typical for the light-front calculation, but to get the standard amplitude this factor has to be divided out (for all external legs) as is done in Eq. (203).

\subsubsection{Vanishing of $\mathcal{M}(-+\cdots+)$ and $\mathcal{M}(++\cdots+)$ on the light front}

It is well known that, the amplitudes for the helicity configurations $(-+\cdots+)$ and $(++\cdots+)$ are vanishing, see discussion in Sec. 3. This property can be proved using supersymmetry relations, as has been done first in [67].

The vanishing of the amplitudes $\mathcal{M}(+,+, \ldots,+)$ on the light-front can, on the other hand, be argued from the angular momentum conservation [77].

The light-front wave functions (as well as the fragmentation functions) satisfy non-trivial conservation of the total angular momentum

$$
J^{z}=\sum_{i=1}^{n} s_{i}^{z}+\sum_{j=1}^{n-1} l_{k}^{z} .
$$

The superscripts $z$ denote the projection along the light-front direction $z$. The first term corresponds to the contribution originating from the spin of the $n$ component Fock state of the wave function in question, whereas the second term is the sum over the relative orbital angular momenta. The second term excludes the contribution from the motion of the center of mass and is only due to the relative motion. The sum rule (204) has been verified for in QED for a systems of electron and spin 1(0) boson [78] and in QCD for dressed quark and gluon [79,80]. For a transition of 2 to $n$ the relative change of the contribution from intrinsic spin is equal to $\Delta S_{z}=n+2$. On the other hand for tree-level $g^{n}$ amplitude there are only $n$ triple gluon vertices, each of which can change the angular momentum by $\Delta l_{z} \pm 1$. Thus the orbital angular momentum can only be changed at most by $\Delta L_{z}=n$. Therefore the amplitude $\mathcal{M}(+,+, \ldots,+)$ needs to vanish on the light-front. Moreover the vanishing has to occur graph by graph in perturbation theory.

The same argument for the helicity flip amplitude $\mathcal{M}(-,+,+, \ldots,+)$ considered above is not sufficient as the requirement (204) can be in principle satisfied for each graphs. Thus the contributions from individual graphs on the light-front do not vanish as we saw in the the simple example above. From Eqs. (179) and (203) we see that the amplitude $\underline{\mathcal{M}}_{1 \rightarrow n}(+\rightarrow+\cdots+)$ is proportional to the energy denominator with the difference of the incoming light-front energies and the outgoing light-front energies. This denominator is non-zero for the case of the off-shell amplitude. However, it vanishes for the on-shell amplitude due to the physical condition. The same result was obtained in [81] using different approach. This corresponds to the vanishing of the on-shell amplitude $\mathcal{M}_{1 \rightarrow n}(+\rightarrow+\cdots+)$, 
or $\mathcal{M}_{2 \rightarrow n}(-+\cdots+)$ which is consistent with the general result obtained from supersymmetry. Note that on the light-front it is only the sum of graphs which vanishes, and not the individual graphs.

\subsection{Berends-Giele recursion relations in the light-front framework}

In order to be able to compute the MHV amplitude for the arbitrary number of external legs on the light-front one needs to utilize some recursion formula. As discussed in the previous sections, the fragmentation functions, which in turn can be related to the off-shell amplitudes, satisfy the factorization property. Therefore this fact that the higher point off-shell amplitudes can be obtained from the amplitudes with less number of external legs will be very useful in computing these helicity amplitudes on the light-front. In Sec. 4.3 it was demonstrated that the $(+\rightarrow+\cdots+)$ fragmentation function satisfies the recursion relation given by Eq. (169). In order to be able to compute the amplitudes with the helicity configuration $(+\rightarrow-+\cdots+)$ one needs to utilize the following more complicated recursion relation

$$
\begin{aligned}
\bar{M}_{1 \rightarrow n+1} & =\sum_{j=2}^{n} V_{+} \sqrt{\frac{z_{1} z_{2} \ldots z_{n+1}}{z_{1 \ldots j} z_{j+1 \ldots n+1}}} T_{j}\left[(1 \ldots j)^{+} \rightarrow 1^{-}, 2^{+}, \ldots, j^{+}\right] \times \\
& \times T_{n+1-j}\left[(j+1 \ldots n+1)^{+} \rightarrow(j+1)^{+}, \ldots,(n+1)^{+}\right] \\
& +\sum_{j=1}^{n} V_{-} \sqrt{\frac{z_{1} z_{2} \ldots z_{n+1}}{z_{1 \ldots j} z_{j+1 \ldots n+1}}} T_{j}\left[(1 \ldots j)^{-} \rightarrow 1^{-}, 2^{+}, \ldots, j^{+}\right] \times \\
& \times T_{n+1-j}\left[(j+1 \ldots n+1)^{+} \rightarrow(j+1)^{+}, \ldots,(n+1)^{+}\right] \\
& +\sum_{j=2}^{n} \sum_{i=1}^{j-1}\left(V_{4}+V_{\text {Coul }}\right) \sqrt{\frac{z_{1} z_{2} \ldots z_{n+1}}{z_{1 \ldots i} z_{i+1 \ldots j} z_{j+1 \ldots n+1}}} T_{i}\left[(1 \ldots i)^{-} \rightarrow 1^{-}, 2^{+}, \ldots, i^{+}\right] \\
& \times T_{j-i}\left[(i+1 \ldots j)^{+} \rightarrow(i+1)^{+}, \ldots, j^{+}\right] \times \\
& \times T_{n+1-j}\left[(j+1 \ldots n+1)^{+} \rightarrow(j+1)^{+}, \ldots,(n+1)^{+}\right] .
\end{aligned}
$$

This recursion relation is schematically depicted in Fig. 23. The first and second sums come from Fig. 23a, Fig. 23b respectively. The third, double sum combines the 4-gluons and the Coulomb term and is depicted in Figs. 23c - 23d. The $V$ 's are the vertex factors and are given by

$$
\begin{aligned}
V_{+} & =2 g z_{1 \ldots n+1} v_{(j+1 \ldots n+1)(1 \ldots j)}^{*}, \\
V_{-} & =2 g z_{j+1 \ldots n+1} v_{(1 \ldots n+1)(1 \ldots j)}, \\
V_{4} & =i g^{2}, \\
V_{\text {Coul }} & =i g^{2} \frac{\left(z_{1 \ldots n+1}+z_{j+1 \ldots n+1}\right)\left(z_{i+1 \ldots j}-z_{1 \ldots i}\right)}{\left(z_{1 \ldots n+1}-z_{j+1 \ldots n+1}\right)^{2}} .
\end{aligned}
$$

Inspecting formula given in Eq. (205) we see that the fragmentation functions involved in the process correspond to three different helicity configurations: $(+\rightarrow+\cdots+),(-\rightarrow-\cdots+),(+\rightarrow-\cdots+)$. Two of these functions were introduced in previous sections $T_{n}\left[(12 \ldots n)^{+} \rightarrow 1^{+}, 2^{+}, \ldots, n^{+}\right]$with expression Eq. (178) and $T_{n}\left[(12 \ldots n)^{-} \rightarrow 1^{-}, 2^{+}, \ldots, n^{+}\right]$with expression Eq. (179).

The third fragmentation function, $T_{n}\left[(12 \ldots n)^{+} \rightarrow 1^{-}, 2^{+}, \ldots, n^{+}\right]$is proportional to the off-shell amplitude $\bar{M}_{1 \rightarrow n+1}(+\rightarrow-+\cdots+)$ as indicated by Eq. (203). Thus, Eq. (205) which is depicted in Fig. 23 and written in terms of the fragmentation functions $T$ can be actually interpreted as a recursion relation for $\bar{M}_{1 \rightarrow n+1}(+\rightarrow-+\cdots+)$.

The above defined recursion relations are light-front equivalents to the Berends-Giele recursion relations [20] discussed in Sec. 3. 


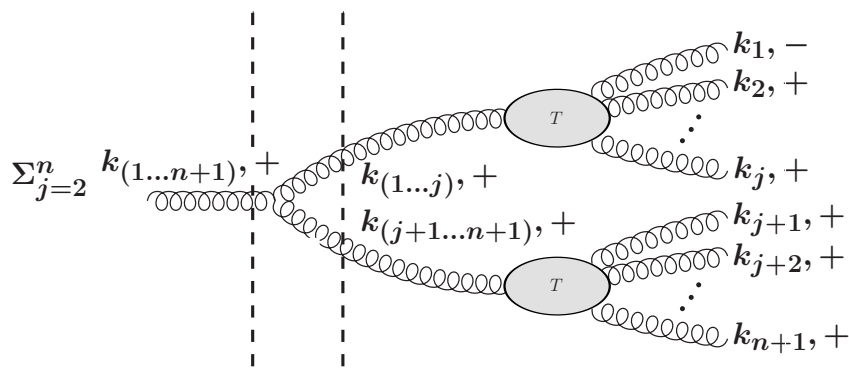

(a)

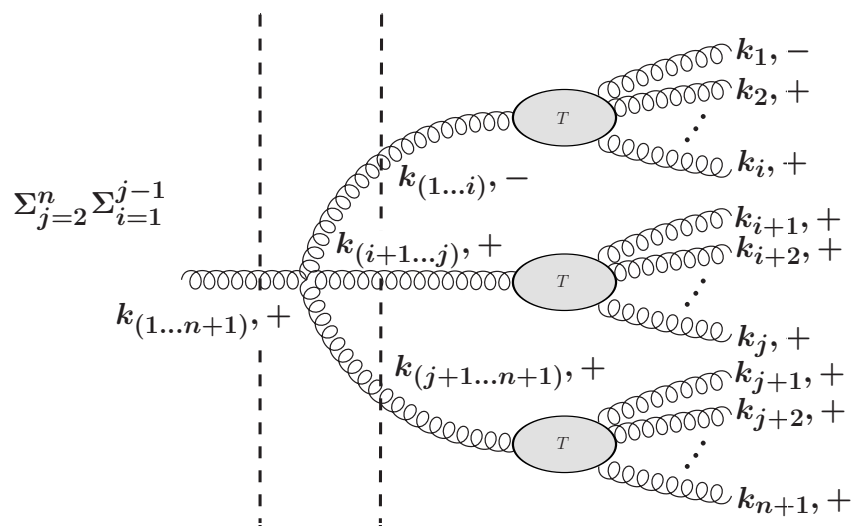

(c)

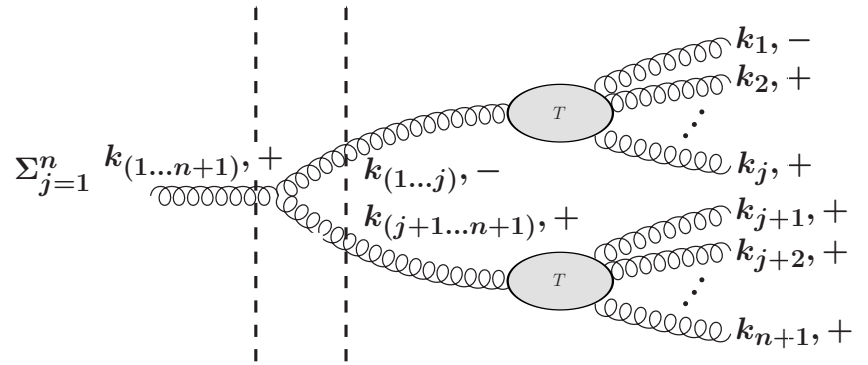

(b)

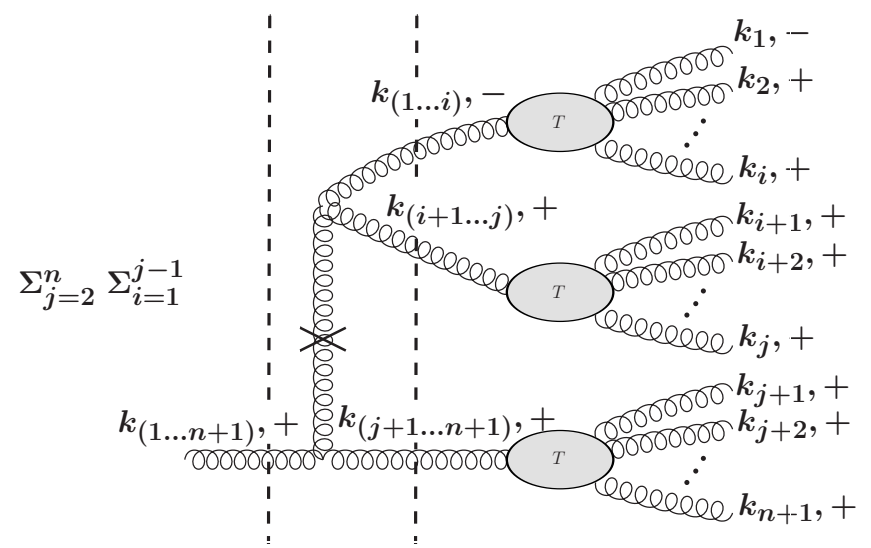

(d)

Figure 23: Graphs involved in the fragmentation of a single off-shell gluon into $n+1$ on-shell gluons. The initial and final helicities are specified in the figures. We denote the 3-gluon vertex in Figs. 23a and $23 \mathrm{~b}$ as $V_{+}$and $V_{-}$respectively, the 4 -gluon vertex in Fig. 23c as $V_{4}$, and the Coulomb term in Fig. $23 \mathrm{~d}$ as $V_{\text {Coul }}$. Vertical lines denote the energy denominators that need to be taken, they are implicit in all intermediate states denoted by blobs. There are no energy denominators in the final state since all the outgoing gluons are on-shell.

Indeed, it turns out that the fragmentation functions $T$ can be related to the gluonic currents which are in the latter recursion. As discussed previously these relations are written for the gauge-dependent current $J^{\mu}$ which is obtained from the amplitudes by taking one of the gluons off-shell. The on-shell subamplitudes can be obtained by the contraction with the appropriate polarization vector and putting the gluon back on-shell, [43]

$$
\mathcal{M}(0,1,2, \ldots, n)=\left.i P^{2} \varepsilon^{\mu} J_{\mu}(1,2, \ldots, n)\right|_{P=-P_{0}},
$$

where we have defined $P=\sum_{i=1}^{n} P_{n}$ and in this formula $p_{i}$ denote the four-vectors for the momenta of the outgoing particles. From the definition of the fragmentation function on the light-front it is clear that one can relate them to the off-shell current in the following way

$$
T_{n}((1,2, \ldots, n) \rightarrow 1,2, \ldots, n) \equiv \varepsilon^{\mu}(12 \ldots n) J_{\mu}(1,2, \ldots, n),
$$

where by $\varepsilon^{\mu}(12 \ldots n)$ we denote the polarization vector of the incoming (off-shell) gluon in the fragmentation function. Now, it is clear that with such definition the factorization property for the fragmentation function (173) is a light-front analog of the Berends-Giele [20] recursion formula, which was presented in Sec. 3. It is somewhat different though in that it contains the Coulomb term (see Fig. 23d) and the corresponding energy denominators, both of which are specific to the light-front formalism. 


\subsection{MHV amplitudes from light-front recursion}

Recursion given in Eq. (205) was used to compute all the MHV amplitudes on the light-front [32]. It turns out that the solution to this recursion can be compactly written in terms of the combination of different types of the off-shell amplitudes. As will be shown in the next sections the new off-shell amplitude that appears is actually gauge invariant and the new recursion relation is identical to the one which involves matrix elements of straight infinite Wilson lines [34]. But before discussing that intriguing relation, let us first show the application of the recursion relation Eq. (205) for the lowest order MHV amplitude.

In order to use the recursion relations first one needs to set the initial conditions. The normalization is such that the initial fragmentation functions are set to

$$
\begin{aligned}
& T_{1}\left[1^{+} \rightarrow 1^{-}\right]=0 \\
& T_{1}\left[1^{+} \rightarrow 1^{+}\right]=1 \\
& T_{1}\left[1^{-} \rightarrow 1^{-}\right]=1
\end{aligned}
$$

The lowest order amplitude is $\underline{\mathcal{M}}_{1 \rightarrow 2}(+\rightarrow-+)$, and so in that case the recursion relation Eq. (205) has to be taken for $n=1$, and in this case only the term coming from graph in Fig. 23b contributes to the amplitude

$$
\underline{\mathcal{M}}_{1 \rightarrow 2}=2 g z_{2} v_{(12) 1} \sqrt{\frac{z_{1} z_{2}}{z_{1} z_{2}}}=2 g \frac{z_{1} z_{12}}{z_{2}} \frac{v_{(12) 1}^{3}}{v_{21} v_{2(12)}}=2 i^{2} g M_{1 \rightarrow 2},
$$

where we defined a new object

$$
M_{1 \rightarrow 2} \equiv \frac{z_{1} z_{12}}{z_{2}} \frac{v_{(12) 1}^{3}}{v_{21} v_{2(12)}}=\frac{z_{1} z_{12}}{z_{2}} \frac{v_{(12) 1}^{4}}{v_{21} v_{2(12)} v_{(12) 1}} .
$$

In order to find $\mathcal{M}_{1 \rightarrow 3}$ one needs to set $n=2$ in the recursion relation given by Eq. (205) and utilize the result (213). Since now all the terms in recursion (205) contribute to this amplitude, the computation of the final result is much more complicated. It turns out that it is convenient to add the terms in the particular order. This reformulation is useful since it can be used in the induction proof to obtain the solution for arbitrary number of external legs. To begin, it will be convenient for the calculations to add $V_{4}$ and $V_{\text {Coul }}$ to get

$$
V_{4}+V_{\text {Coul }}=V_{\text {comb, a }}+V_{\text {comb, } \mathrm{b}}
$$

where

$$
\begin{gathered}
V_{\text {comb }, \mathrm{a}}=2 i g^{2} \frac{z_{1 \ldots n+1} z_{i+1 \ldots j}}{z_{1 \ldots j}^{2}}, \\
V_{\text {comb }, \mathrm{b}}=-2 i g^{2} \frac{z_{1 \ldots i} z_{j+1 \ldots n+1}}{z_{1 \ldots j}^{2}} .
\end{gathered}
$$

Thus, one can replace the contributions from the 4-gluon vertex and the Coulomb term shown in Figs. $23 \mathrm{c}$ and $23 \mathrm{~d}$ by two other effective 4 -gluon interactions. The recursion (205) gives five different terms 
which contribute to $\underline{\mathcal{M}}_{1 \rightarrow 3}$ :

$$
\begin{aligned}
& \mathrm{I}=2 g z_{123} v_{(3)(12)}^{*} \sqrt{\frac{z_{1} z_{2} z_{3}}{z_{12} z_{3}}}\left[\frac{1}{\sqrt{z_{12} z_{1} z_{2}}} \frac{i}{D_{2}} \frac{\left.\mathcal{M}_{1 \rightarrow 2}\right]}{\mathrm{II}}=2 g z_{23} v_{(123) 1} \sqrt{\frac{z_{1} z_{2} z_{3}}{z_{1} z_{23}}}\left[(-i g)^{1}\left(\frac{z_{23}}{z_{2} z_{3}}\right)^{3 / 2} \frac{1}{v_{32}}\right]=-2 i g^{2} \frac{z_{23}^{2}}{z_{2} z_{3}} \frac{v_{(321) 1}}{v_{32}}\right. \\
& \mathrm{III}=2 g z_{3} v_{(123)(12)} \sqrt{\frac{z_{1} z_{2} z_{3}}{z_{12} z_{3}}}\left[(-i g)^{1}\left(\frac{z_{1}}{z_{12}}\right)^{2}\left(\frac{z_{12}}{z_{1} z_{2}}\right)^{3 / 2} \frac{1}{v_{21}}\right]=-2 i g^{2} \frac{z_{1} z_{3}}{z_{12} z_{2}} \frac{v_{(321)(21)}}{v_{21}} \\
& \mathrm{IV}=2 i g^{2} \frac{z_{123} z_{2}}{z_{12}^{2}} \sqrt{\frac{z_{1} z_{2} z_{3}}{z_{1} z_{2} z_{3}}}=-2 i g^{2} \frac{z_{123} z_{3}}{z_{12}^{2} z_{3}} \frac{M_{1 \rightarrow 2}}{v_{(12) 1}} \\
& \mathrm{~V}=-2 i g^{2} \frac{z_{1} z_{3}}{z_{12}^{2}} \sqrt{\frac{z_{1} z_{2} z_{3}}{z_{1} z_{2} z_{3}}} .
\end{aligned}
$$

Here, contributions from the two effective 4-gluon vertices have been written as IV and V. The sum of terms II+III+V can be written as

$$
-2 i g^{2} v_{(123) 1}\left\{\frac{M_{1 \rightarrow 3}}{v_{(123) 1}}-\frac{1}{z_{3}} \frac{z_{123}^{2}}{z_{12} z_{123}} \frac{1}{v_{3(123)}} \frac{M_{1 \rightarrow 2}}{v_{(12) 1}}\right\} .
$$

Thus we see from the above term as well as the rest of the contributions, that the amplitude $\underline{\mathcal{M}}_{1 \rightarrow 3}$ is written completely in terms of the new objects $M_{1 \rightarrow 2}$ and $M_{1 \rightarrow 3}$. In the above expression we have introduced a new object

$$
M_{1 \rightarrow 3}=\frac{z_{123} z_{1}}{z_{2} z_{3}} \frac{v_{(123) 1}^{3}}{v_{12} v_{23} v_{3(123)}} .
$$

Rearranging the terms we find the following expressions for the off-shell amplitude $\underline{\mathcal{M}}_{1 \rightarrow 3}$,

$$
\underline{\mathcal{M}}_{1 \rightarrow 3}=2 i^{3} g^{2} M_{1 \rightarrow 3}-2 i^{3} g^{2} \frac{1}{z_{3}} \frac{z_{123}}{z_{12}} \frac{1}{v_{3(123)}} \frac{D_{3}}{D_{2}} M_{1 \rightarrow 2},
$$

where the following useful relation has been used

$$
2 z_{3} z_{123} v_{3(12)}^{*} v_{3(123)}=-z_{3} z_{12}\left(\frac{\vec{k}_{3}}{z_{3}}-\frac{\vec{k}_{12}}{z_{12}}\right)^{2}=z_{123}\left(D_{2}-D_{3}\right),
$$

It is evident that the new pattern emerges where the off-shell amplitude can be expressed as a combination of objects with lower number of external legs. These new amplitudes are special cases (for $n=2, n=3$ ) of the general definition for arbitrary number $n$ of particles

$$
M_{1 \rightarrow n} \equiv \frac{z_{1 \ldots n} z_{1}}{z_{2} z_{3} \ldots z_{n}} \frac{v_{(1 \ldots n) 1}^{3}}{v_{12} v_{23} \ldots v_{n-1 n} v_{n(1 \ldots n)}},
$$

This object is for off-shell kinematics, yet remarkably, it has exactly the same form (up to some multiplicative constant factors) as the on-shell MHV scattering amplitude for $2 \rightarrow n-1$ transition, $\mathcal{M}_{2 \rightarrow n-1}$, see Eq. 120. The amplitude $\mathcal{M}_{1 \rightarrow 4}$, can be found by following the same procedure, that is by reusing the result for the $\underline{\mathcal{M}}_{1 \rightarrow 3}$. It is however much more cumbersome and longer process therefore we shall only quote here the final result for the $1 \rightarrow 4$ transition

$$
\underline{\mathcal{M}}_{1 \rightarrow 4}=2 i^{4} g^{3}\left\{M_{1 \rightarrow 4}-\frac{D_{4}}{D_{3}} \frac{z_{1234}^{2}}{z_{123} z_{1234}} \frac{1}{z_{4}} \frac{1}{v_{4(1234)}} M_{1 \rightarrow 3}-\frac{D_{4}}{D_{2}} \frac{z_{1234}^{2}}{z_{12} z_{123}} \frac{1}{z_{3} z_{4}} \frac{1}{v_{34}} \frac{1}{v_{3(123)}} M_{1 \rightarrow 2}\right\} .
$$


Interestingly, the off-shell amplitude is expressed as a linear combination of the objects which have the form of the on-shell MHV amplitudes with the pre factors which are proportional to the energy denominators. In particular we see that by putting the on-shell constraint $D_{4}=0$ we recover the on-shell amplitude

$$
\left.\underline{\mathcal{M}}_{1 \rightarrow 4}\right|_{D_{4}=0}=\left.2 i^{4} g^{3} M_{1 \rightarrow 4}\right|_{D_{4}=0}=2 i^{4} g^{3} \frac{z_{123} z_{1}}{z_{2} z_{3}} \frac{v_{(123) 1}^{3}}{v_{12} v_{23} v_{3(123)}},
$$

which is exactly equal to the MHV result Eq. (120). Therefore the light-front result is consistent with the results previously obtained in the literature using more standard methods of perturbation theory.

Following the pattern found, one would then expect, for a general integer $n \geq 2$,

$$
\underline{\mathcal{M}}_{1 \rightarrow n}^{(+\rightarrow-+\cdots+)}=2 i^{n} g^{n-1}\left\{M_{1 \rightarrow n}-z_{1 \ldots n}^{2} D_{n} \sum_{i=2}^{n-1} \frac{1}{z_{1 \ldots i} z_{1 \ldots i+1}} \frac{1}{z_{i+1} \ldots z_{n}} \frac{1}{v_{i+1} i+2 \ldots v_{n-1 n}} \frac{M_{1 \rightarrow i}}{v_{i+1(1 \ldots i+1)} D_{i}}\right\} .
$$

Indeed this formula was found in [32] first by computing the lowest order few terms and then performing proof by mathematical induction. A curious fact about $\underline{\mathcal{M}}^{(+\rightarrow-+\ldots+)}$ is that it is written entirely as a sum of amplitudes $M^{(+\rightarrow-+\ldots+)}$ with different number of legs. From the point of view of the helicity structure it is perhaps expected that there should be contributions from $M^{(+\rightarrow-+\ldots+)}$. On the other hand looking at Fig. 23, it is not immediately obvious as to why $M^{(+\rightarrow-+\ldots+)}$ should dominate the expression since only Fig. 23a has a subamplitude with helicity configuration $(+\rightarrow-+\ldots+)$, the other graphs contain either 4 -gluon, Coulomb contribution or the yet another structure $-\rightarrow-+\cdots+$. It turns out that the other graphs, even though their substructures have different helicity configuration, do contribute terms exactly proportional to $M^{(+\rightarrow-+\ldots+)}$. Writing out the these terms explicitly and through an involved algebraic manipulation, it can finally be shown [32] that the relation (230) indeed solves the recursion given by Eq. (205) on the light-front.

The new recursion relation (230) can be rewritten in a more elegant way. In order to do that let us inspect the second term in Eq. (230). One can show that it can be expressed as the sum over the products

$$
\mathcal{M}^{(+\rightarrow+\cdots+)}\left(k_{(1 \ldots i) i+1 \ldots N}\right) \frac{1}{z_{1 \ldots i} D_{(1, \ldots, i)}} M^{(+\rightarrow-+\cdots+)}\left(k_{1 \ldots i}\right)
$$

Let us start with the definition of the off-shell subamplitude for the helicity configuration $(+\rightarrow+\cdots+)$ which is given by (203) together with (178)

$$
\begin{aligned}
\underline{\mathcal{M}}^{(+\rightarrow+\cdots+)}\left(k_{(1 \ldots i) i+1 \ldots N}\right) & = \\
& -i^{N-i+1} g^{N-i} D_{((1 \ldots i), i+1, \ldots, N)} \frac{z_{1 \ldots N}^{2}}{z_{1 \ldots i} z_{i+1} \ldots z_{N}} \frac{1}{v_{(1 \ldots i) i+1} v_{i+1} i+2 \ldots v_{N-1 N}} .
\end{aligned}
$$

The denominator in this expression is equal to

$$
D_{((1 \ldots i), i+1, \ldots, N)}=E_{1 \ldots i}^{-}+\sum_{j=i+1}^{N} E_{j}^{-}-E_{1 \ldots N}^{-}=\frac{\vec{k}_{1 \ldots i \perp}^{2}}{z_{1 \ldots i}}+\sum_{j=i+1}^{i} \frac{\vec{k}_{j \perp}^{2}}{z_{j}}-\frac{\vec{k}_{1 \ldots N \perp}^{2}}{z_{1 \ldots N}} .
$$

Note that (232) vanishes in the on-shell limit $D_{((1 \ldots i), i+1, \ldots, N)}=0$, which is consistent with the fact that the on-shell amplitude vanishes for this helicity configuration $(+\rightarrow+\cdots+)$. Comparing with (230), we see that the term inside the sum is very similar to the above off-shell subamplitude with some additional prefactors.

Substituting (232) in (230) and using following identity

$$
z_{1 \ldots i+1} v_{(i+1)(1 \ldots i+1)}=z_{1 \ldots i} v_{(i+1)(1 \ldots i)}
$$




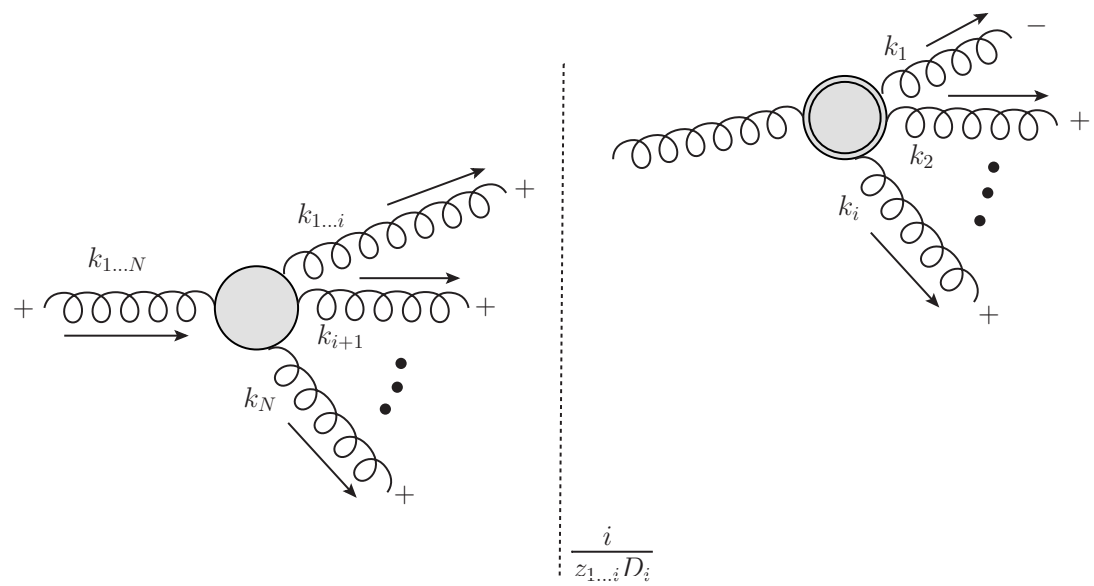

Figure 24: Schematic representation of the second term in the new recursion formula (235). The dotted vertical line represents the energy denominator $i / z_{1 \ldots i} D_{i}$, the graph on the left hand side of this line is the amplitude $\underline{\mathcal{M}}^{(+\rightarrow+\cdots+)}\left(k_{(1 \ldots i) i+1 \ldots N}\right)$ whereas the graph on the right hand side is the amplitude $M^{(+\rightarrow-+\cdots+)}\left(k_{1 \ldots i}\right)$. The double line around the blob in the latter graph indicates that this is $M$ (with an explicit form of 227) rather than $\mathcal{M}$ which are different objects as explained in the text.

one can finally obtain the following expression

$$
\begin{aligned}
\mathcal{M}^{(+\rightarrow-+\cdots+)}\left(k_{1 \ldots N}\right) & =M^{(+\rightarrow-+\cdots+)}\left(k_{1 \ldots N}\right) \\
+ & \sum_{i=2}^{N-1} \frac{D_{(1, \ldots, N)}}{D_{((1 \ldots i), i+1, \ldots, N)}} \mathcal{M}^{(+\rightarrow+\cdots+)}\left(k_{(1 \ldots i) i+1 \ldots N}\right) \frac{i}{z_{1 \ldots i} D_{(1, \ldots, i)}} M^{(+\rightarrow-+\cdots+)}\left(k_{1 \ldots i}\right) .
\end{aligned}
$$

One can observe that the second term on the right hand side of this recursion has a nice factorized form which can be recast diagrammatically as in Fig. 24. It consists of the sum over the factorized products of the off-shell amplitudes $M$ and $\underline{\mathcal{M}}$. $\underline{\mathcal{M}}$, however, is evaluated with a different denominator in the sense that the ratio $D_{(1, \ldots, N)} / D_{((1 \ldots i), i+1, \ldots, N)}$ cancels the energy denominator inside of $\underline{\mathcal{M}}$ and replaces it with $D_{(1, \ldots, N)}$. It was demonstrated in [33] that the above recursion relation has its roots in the recurrence property of the straight infinite Wilson line, which corresponds to the amplitude $M$.

\subsection{Wilson lines and gauge invariance of the off-shell amplitudes}

Recently a significant progress has been made in the construction the off-shell amplitudes which satisfy gauge invariance [71-73, 82, 83]. A systematic approach to the off-shell amplitudes using Wilson lines was developed in [34]. There, the off-shell gauge invariant amplitude was constructed using the matrix element of a certain Wilson line operator. Such object is gauge invariant, i.e. it satisfies the Ward identities with respect to all on-shell legs. For that object one can write the recurrence relation that is very similar to the one obtained on the light-front. In fact, one can directly prove that for the simplest non-trivial combination of the helicities both recursion relations are exactly identical.

In order to demonstrate the origin of this recursion relation et us recall some basic facts about the gluon Green's function and Ward identities. One considers Green's function in momentum space, at the tree level, with the external momenta $k_{1 \ldots N}, k_{1}, k_{2}, \ldots, k_{N}$. Here the notation is such that $k_{i}$ denote full 4 -momenta and $k_{1 \ldots N}$ is incoming and the rest of the particles are outgoing. Such gluon Green's function is schematically depicted in Fig. 25, all the external particles are considered to be off-shell, the blobs at the end of the gluon lines indicate that the propagators are not amputated and Lorentz indices are not contracted. The on-shell scattering amplitude can be constructed by amputating the propagators, 
setting the momenta to the on-shell limit and contracting with the appropriate polarization vectors which should be transverse to the momenta.

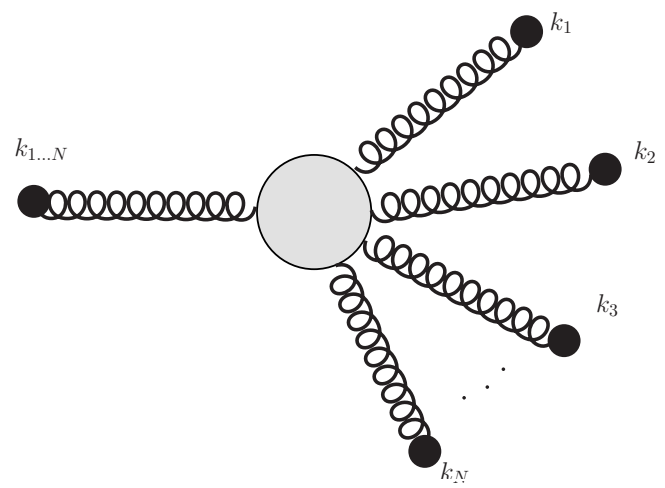

Figure 25: Schematic representation of the gluonic Green's function. The blobs at the end of the gluon lines indicate that the propagators have not been amputated.

For the purpose of making the connection to the light-front recursion formula, and the off-shell amplitudes that appear there, i.e. $\underline{\mathcal{M}}$, one needs however the off-shell amplitude. To be precise, one considers the situation when the incoming particle with the momentum $k_{1 \ldots N}$ is off-shell and the outgoing particles with momenta $k_{1}, \ldots, k_{n}$ are taken on-shell. The off-shell amplitude then is defined by the procedure described above, with the difference that $k_{1 \ldots N}$ is kept off-shell and the off-shell leg is constructed with the polarization vector $\mathfrak{e}_{1 \ldots N}$ which is transverse to the momentum $k_{1 \ldots N}$. Using the currents introduced before in Sec. 3.3, which can be obtained from the Green's function, we can write ${ }^{1}$

$$
\mathcal{M}\left(\mathfrak{e}_{1 \ldots N}, \varepsilon_{1}, \ldots, \varepsilon_{N}\right)=k_{1 \ldots N}^{2} \mathfrak{e}_{1 \ldots N}^{\mu} J_{\mu}\left(\varepsilon_{1}, \ldots, \varepsilon_{N}\right) .
$$

The off-shell amplitude constructed in the way, is not gauge invariant, and this can be checked by replacing the polarization vector with momentum and verifying that indeed

$$
\mathcal{M}\left(\mathfrak{e}_{1 \ldots N} ; \varepsilon_{1}, \ldots, k_{i}, \ldots \varepsilon_{N}\right) \neq 0, \text { for }, \quad i=1, \ldots, N
$$

This statement is also valid for the currents. In particular the off-shell currents which appear in the Berends-Giele formula are not gauge invariant. It turns out however that one can define the gauge invariant extension of this amplitude.

For example, in analysis of scattering at high-energy, i.e. in the Regge limit $s \gg|t|$ within the perturbative QCD, one encounters similar objects. There, the $\mathfrak{e}_{1 \ldots N}$ is set to one of the light-cone components $n_{ \pm}\left(n_{ \pm}^{2}=0\right)$ of a hadron momentum and $k_{1 \ldots N}=x n_{ \pm}+k_{T}$, so that $k_{1 \ldots N} \cdot n_{ \pm}=0$. The gauge invariant vertices corresponding to transitions of such off-shell gluons to a set of on-shell gluons can be derived from the so-called Lipatov's effective action $[84,85]$.

The gauge invariant extension of the off-shell amplitude was considered in [34], where a general construction was presented for amplitudes with multiple off-shell legs. Here, we shall limit ourselves to the case of one off-shell leg. This generalized amplitude is built by considering a matrix element of a straight infinite Wilson line operator of the following form

$$
\begin{aligned}
& \mathfrak{M}_{\mathfrak{e}_{1 \ldots N}}^{a_{1 \ldots N} a_{1} \ldots a_{N}}\left(k_{1 \ldots N} ; k_{1}, \ldots, k_{N}\right)=\int d^{4} x e^{i k_{1 \ldots N} \cdot x} \\
& \left\langle 0\left|\mathcal{T}\left\{R_{\mathfrak{e}_{1} \ldots N}^{a_{1 \ldots N}}(x) e^{i S_{\mathrm{Y}-\mathrm{M}}}\right\}\right| k_{1}, \lambda_{1}, a_{1} ; \ldots ; k_{N}, \lambda_{N}, a_{N}\right\rangle_{c},
\end{aligned}
$$

\footnotetext{
${ }^{1}$ From here on, we have changed the notation slightly to avoid the proliferation of too many symbols which denote different amplitudes. The amplitude denoted by $\mathcal{M}$ can be now on-shell or off-shell.
} 
with

$$
R_{\mathfrak{e}_{1 \ldots N}}^{a_{1 \ldots N}}(x)=\operatorname{Tr}\left[t^{a_{1 \ldots N}} \mathcal{P} \exp \left(i g \int_{-\infty}^{+\infty} d s A_{\mu}^{b}\left(x+s \mathfrak{e}_{1 \ldots N}\right) \mathfrak{e}_{1 \ldots N}^{\mu} t^{b}\right)\right],
$$

where $\mathcal{T}$ is the time-ordering, $\mathcal{P}$ is the path-ordering, $S_{\text {Y-M }}$ is the Yang-Mills interaction action, and, finally, $\left|k_{i}, \lambda_{i}, a_{i}\right\rangle$ are one-gluon on-shell states with momentum $k_{i}$, helicity $\lambda_{i}$ and color $a_{i}$. The color index for the off-shell gluon field is $a_{1 \ldots N}$. The subscript $c$ means that only connected contributions are included. The infinite Wilson line operator $R_{\mathfrak{e}_{1} \ldots N}^{a_{1} \ldots N}$ sandwiched in the matrix element is explicitly gauge invariant with respect to small gauge transformations. In Ref. [34], instead of a straight infinite path in (239), deformed paths were considered in order to regularize the integrals and it was shown that they form certain generalized functions.

Let us consider the color ordered version of the matrix element defined in (238) with the order of color indices equal to $\left(a_{1 \ldots N}, a_{1}, \ldots, a_{N}\right)$.

It was shown in [34] that it is proportional to the standard Dirac delta which guarantees momentum conservation and another Dirac delta which enforces that the momentum of the off-shell gluon $k_{1 \ldots N}$ and the vector $\mathfrak{e}_{1 \ldots N}$ are mutually transverse

$$
\mathfrak{M}_{\mathfrak{e}_{1} \ldots N}\left(k_{1 \ldots N}\right)=\delta^{(4)}\left(k_{1 \ldots N}-k_{1}-\ldots-k_{N}\right) \delta\left(\mathfrak{e}_{1 \ldots N} \cdot k_{1 \ldots N}\right) \tilde{\mathcal{M}}_{\mathfrak{e}_{1} \ldots N}^{\left(\lambda_{1} \ldots \lambda_{N}\right)}\left(k_{1 \ldots N}\right)
$$

where we have used the shorthand notation for the arguments of the amplitudes:

$$
\mathcal{M}\left(k_{1 \ldots j} ; k_{1 \ldots i}, k_{i+1}, \ldots, k_{j}\right) \equiv \mathcal{M}\left(k_{(1 \ldots i) i+1 \ldots j}\right) .
$$

This amplitude corresponds to the process $k_{1 \ldots j} \rightarrow k_{1 \ldots i}+k_{i+1}+\cdots+k_{j}$. For example, we denote $\mathcal{M}^{(+\rightarrow+++)}\left(k_{123} ; k_{(12)}, k_{3}\right)$ as $\mathcal{M}^{(+\rightarrow+++)}\left(k_{(12) 3}\right)$.

The relation represented by Eq. (240) defines new amplitude $\tilde{\mathcal{M}}$ which is gauge invariant off-shell amplitude which has helicities $\lambda_{1}, \ldots, \lambda_{N}$ for the on-shell gluons and the "polarization" vector $\mathfrak{e}_{1 \ldots N}$ for the off-shell gluon. The amplitude $\tilde{\mathcal{M}}$ is gauge invariant since the Ward identities are satisfied for all on-shell legs [34] (but not with respect to the Wilson line slope). To be precise one has

$$
\tilde{\mathcal{M}}_{\mathfrak{e}_{1} \ldots N}^{\left(\lambda_{1} \ldots k_{i} \ldots \lambda_{N}\right)}\left(k_{1 \ldots N}\right)=0 \text { for } i=1, \ldots, N .
$$

Above, the $k_{i}$ in the argument for helicities means that the amplitude has been contracted with the momentum vector $k_{i}$ in place of the polarization vector. Let us stress that the amplitude $\tilde{\mathcal{M}}$ is indeed gauge invariant only when $k_{1 \ldots N} \cdot \mathfrak{e}_{1 \ldots N}=0$.

Diagrammatically, one can draw the following expression for the amplitude $\tilde{\mathcal{M}}$

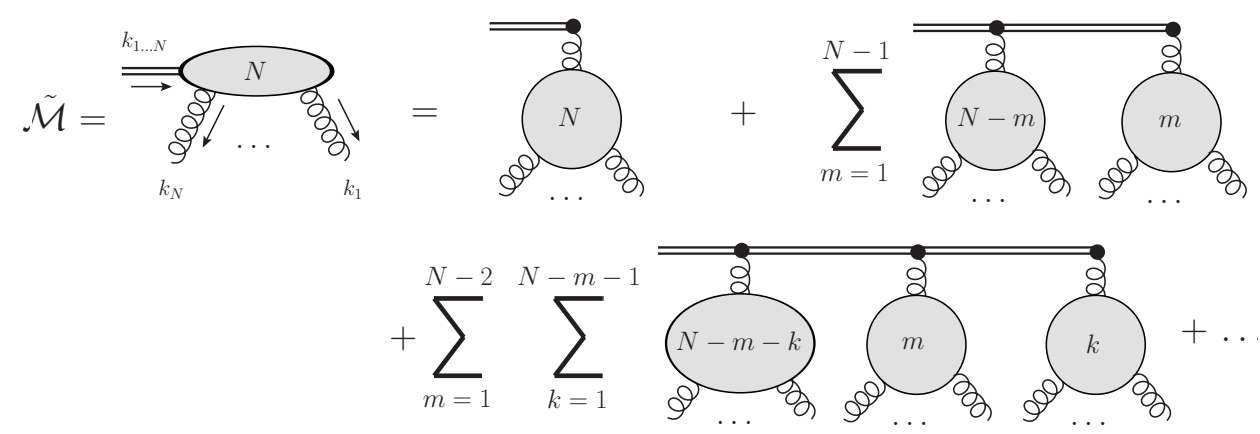

The double line in above figure represents the Wilson line in momentum space. Each Wilson line which is between two gluon attachments contributes the propagator $i / p \cdot \mathfrak{e}_{1 \ldots N}$, with $p$ being the momentum flowing through the line. The gluons couple to the Wilson line via an $i g \mathfrak{e}_{1 \ldots N}^{\mu}$ vertex. The details on the Feynman rules with Wilson lines can be found in [34]. The shaded blobs represent standard QCD amplitudes and the letters in the blobs are indicating the number of external on-shell legs. The 
first contribution on the right hand side in (243) is the off-shell amplitude defined in (236) (modulo $i g$ factor due to a coupling with the gauge link). In what follows we will denote this amplitude as $\overline{\mathcal{M}}^{\left(\mathfrak{e}_{1} \ldots N \rightarrow \lambda_{1} \ldots \lambda_{N}\right)}\left(k_{1 \ldots N}\right)$. It will contain the off-shell propagator and and a coupling to the Wilson line

$$
\overline{\mathcal{M}}^{\left(\mathfrak{e}_{1} \ldots N \rightarrow \lambda_{1} \ldots \lambda_{N}\right)}\left(k_{1, \ldots, N}\right)=i g \frac{-i}{k_{1 \ldots N}^{2}} i \mathcal{M}^{\left(\mathfrak{e}_{1} \ldots N \rightarrow \lambda_{1} \ldots \lambda_{N}\right)}\left(k_{1, \ldots, N}\right),
$$

where $\mathcal{M}$ is the standard QCD amplitude calculated from Feynman diagrams (with, however, off-shell kinematics). Let us underline one more time that the amplitude $\mathcal{M}$ (or $\overline{\mathcal{M}}$ ) itself does not satisfy the Ward identities, but they are restored thanks to the rest of the r.h.s of Eq. (243).

In short the Eq. (243) can be schematically rewritten as

$$
\tilde{\mathcal{M}}=\overline{\mathcal{M}}+\text { terms restoring gauge invariance . }
$$

The decomposition (243) can be rewritten in a more compact form by means of the following recursion

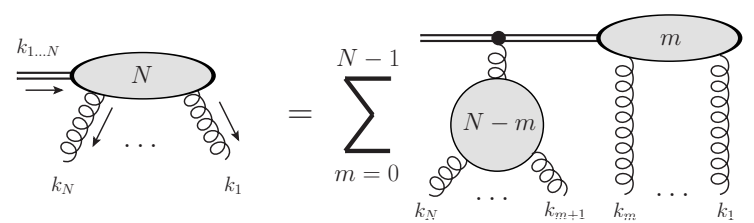

The algebraic form of this recursion relation can be written as [33]

$$
\tilde{\mathcal{M}}_{\mathfrak{e}_{1} \ldots N}^{\left(\lambda_{1} \ldots \lambda_{N}\right)}\left(k_{1 \ldots N}\right)=\sum_{m=0}^{N-1} \tilde{\mathcal{M}}_{\mathfrak{e}_{1} \ldots N}^{\left(\lambda_{1} \ldots \lambda_{m}\right)}\left(k_{1 \ldots m}\right) \frac{1}{k_{1 \ldots m} \cdot \varepsilon_{1 \ldots N}} \overline{\mathcal{M}}^{\left(\mathfrak{e}_{1 \ldots N} \rightarrow \lambda_{m+1} \ldots \lambda_{N}\right)}\left(k_{m+1, \ldots, N}\right),
$$

with

$$
\tilde{\mathcal{M}}_{\mathfrak{e}_{1 \ldots N}}^{\left(\lambda_{i}\right)}\left(k_{i}\right)=i^{2} g \mathfrak{e}_{1 \ldots N}^{*} \cdot \varepsilon_{i}^{\lambda_{i}}
$$

and $\tilde{\mathcal{M}}_{\mathfrak{e}_{1} \ldots N}^{\left(\lambda_{1} \ldots \lambda_{m}\right)}\left(k_{1 \ldots m}\right)=1$ for $m=0$. Let us emphasize an important difference between the amplitudes $\tilde{\mathcal{M}}$ which appear on both sides of Eq. (247). The amplitude on the left hand side of (247) satisfies property (242) and thus is gauge invariant. On the contrary, the amplitude $\tilde{\mathcal{M}}$ which appears on the right hand side of (247) is not gauge invariant. This stems from the fact that the replacement $\varepsilon_{i} \leftrightarrow k_{i}$ will lead to the non-vanishing result

$$
\tilde{\mathcal{M}}_{\mathfrak{e}_{1} \ldots N}^{\left(\lambda_{1} \ldots k_{i} \ldots \lambda_{m}\right)}\left(k_{1 \ldots m}\right) \neq 0 \text { for } i=1, \ldots, m .
$$

This is because the Wilson line slope defining $\tilde{\mathcal{M}}$ is not perpendicular to the off-shell momentum, $\mathfrak{e}_{1 \ldots N} \cdot k_{1 \ldots m} \neq 0$, as required by $(242)$.

\subsection{Relation of Wilson lines to the light-front ampliudes}

The recursion (247) illustrated in (246) relates two amplitudes $\tilde{\mathcal{M}}$, each of them having different number of external legs. That means that the object on the left hand side of this recursion can be obtained from the amplitude with lower number of legs. This is similar to the recursion (230) obtained within the lightfront formalism and therefore we shall show that it is possible to relate both recursions. It is tempting to identify immediately the amplitude $\tilde{\mathcal{M}}$ on the right hand side of Eq. (247) with the amplitude $M$ on the right-hand side of (230). As mentioned earlier the two objects which are on two sides of the recursion (247) are actually different as the one is gauge invariant and the other isn't. Therefore we need to find the transformation which will allow us to compare directly the two recursions. 
To this aim, we first have to choose the appropriate "polarization" vector $\mathfrak{e}_{1 \ldots N}$ for the off-shell gluon. One has to choose the same vector as in the light-front formalism to obtain (230)

$$
\mathfrak{e}_{1 \ldots N}^{\mu}=\varepsilon_{1 \ldots N}^{+\mu},
$$

where $\varepsilon_{1 \ldots N}^{+}$is defined by (53). We note that $\varepsilon_{1 \ldots N} \cdot k_{1 \ldots N}=0$, and $\tilde{\mathcal{M}}_{\varepsilon_{1 \ldots N}^{+}}^{\left(\lambda_{1} \ldots \lambda_{N}\right)}\left(k_{1 \ldots N}\right)$ is gauge invariant despite the fact that $k_{1 \ldots N}$ is off-shell. Since the light-front recursion was derived for $+\rightarrow-+\cdots+$ configuration of helicities one has to choose the same helicities, that is $\lambda_{1}=-, \lambda_{2}=\cdots=\lambda_{N}=+$ and the reference momenta for the polarization vectors are chosen to be equal to vector $\eta$. Using these substitutions one can write $(247)$ as

$$
\begin{aligned}
\tilde{\mathcal{M}}_{\varepsilon_{1 \ldots N}^{+}}^{(-+\cdots+)}\left(k_{1 \ldots N}\right)=\overline{\mathcal{M}}^{(+\rightarrow-\cdots+)}\left(k_{1, \ldots, N}\right) & \\
& +\sum_{m=2}^{N-1} \tilde{\mathcal{M}}_{\varepsilon_{1 \ldots N}^{+}}^{(-+\cdots+)}\left(k_{1 \ldots m}\right) \frac{1}{\tilde{v}_{(1 \ldots m)(1 \ldots N)}} \overline{\mathcal{M}}^{(+\rightarrow+\cdots+)}\left(k_{m+1, \ldots, N}\right) .
\end{aligned}
$$

Note that now the sum starts with the index $m=2$. For $m=1$ the term vanishes since according to Eq. (248) it is proportional to

$$
\mathfrak{e}_{1 \ldots N}^{*} \cdot \varepsilon_{1}^{-}=\varepsilon_{1 \ldots N}^{+*} \cdot \varepsilon_{1}^{-}=0 .
$$

In Eq. (251) new variable was introduced $\tilde{v}_{i j}$ defined as

$$
\tilde{v}_{i j}=\varepsilon_{j}^{-} \cdot k_{i}=k_{i}^{+} v_{j i},
$$

Relation (251) involves two amplitudes for two different sets of helicities, Therefore one first needs to compute the off-shell amplitude for the $+\rightarrow+\cdots+$ configuration and then this object can be used in the recursion above to compute the other amplitude. Thus in order to proceed, we have to find an explicit expression for the off-shell amplitude $\overline{\mathcal{M}}^{(+\rightarrow+\cdots+)}$. This can be done using the recursion (247), but now with the different choice of helicities, i.e. $\lambda_{1}=\cdots=\lambda_{N}=+$ and observing that

$$
\tilde{\mathcal{M}}_{\varepsilon_{1 \ldots N}^{+}}^{(+\cdots+)}=0 .
$$

This of course is related to the fact that the on-shell amplitude, which is obviously gauge invariant, vanishes for this set of helicities, see Sec. 3. The result for amplitude $+\rightarrow+\cdots+$ obtained from solving the Wilson line recursion [33] reads

$$
\overline{\mathcal{M}}^{(+\rightarrow+\cdots+)}\left(k_{1, \ldots, N}\right)=-g^{N} \frac{\tilde{v}_{(1 \ldots N) 1}}{\tilde{v}_{1(1 \ldots N)}} \frac{1}{\tilde{v}_{N(N-1)} \ldots \tilde{v}_{32} \tilde{v}_{21}} .
$$

It is quite remarkable that this expression is identical to the one obtained from the light-front approach (232), even though the two are (gauge dependent) off-shell amplitudes obtained within the two different frameworks. However, even with this encouraging result, the recursion (251) is still rather different then the one obtained within the light-front formulation. Indeed, this recursion does not involve the same object on the l.h.s and r.h.s of the equation, as we already discussed below equation (247). The recursion relation (251) can be, however, written entirely in terms of gauge invariant off-shell amplitudes. That means that indeed one can find the kernel $K_{m N}$ such that

$$
\begin{aligned}
\tilde{\mathcal{M}}_{\varepsilon_{1 \ldots N}^{+}}^{(-+\cdots+)}\left(k_{1 \ldots N}\right)=\overline{\mathcal{M}}^{(+\rightarrow-\cdots+)}\left(k_{1, \ldots, N}\right) & \\
& +\sum_{m=2}^{N-1} \tilde{\mathcal{M}}_{\varepsilon_{1 \ldots m}^{+}}^{(-+\cdots+)}\left(k_{1 \ldots m}\right) K_{m N} \overline{\mathcal{M}}^{(+\rightarrow+\cdots+)}\left(k_{m+1, \ldots, N}\right) .
\end{aligned}
$$


Let us underline the crucial difference with relation (251). Now, on the r.h.s., we encounter $\tilde{\mathcal{M}}_{\varepsilon_{1 \ldots m}^{+}}^{(-+\cdots+)}\left(k_{1 \ldots m}\right)$, which does satisfy the Ward identities since $\varepsilon_{1 \ldots m}^{+} \cdot k_{1 \ldots m}=0$. On the contrary, in (251) we had $\tilde{\mathcal{M}}_{\varepsilon_{1 \ldots N}^{+}}^{(-+\cdots+)}\left(k_{1 \ldots m}\right)$ (note the different Wilson line slope here), which is not gauge invariant, as discussed below equation (247).

The kernel $K_{m N}$ was derived in [33] after very non-trivial algebraic manipulations, but it turns out that remarkably it has a very compact form

$$
K_{m N}=\frac{z_{1 \ldots N}}{z_{m+1 \ldots N} \tilde{v}_{(1 \ldots m+1)(m+1)}}
$$

It is straightforward to check that (256) with (257) and (255) coincides exactly with (230) obtained within the light-front approach. To this end one only needs to redefine $\mathcal{M}$ and $M$ in (230) to include the energy denominators with appropriate $z_{i}$ 's forming in fact propagators. This means also, that the off-shell amplitude $M$ in (230) which has the form of the MHV amplitude is indeed gauge invariant since it corresponds to the gauge invariant $\tilde{\mathcal{M}}$ from the Wilson line approach. Similar observation about the off-shell amplitudes was made in the work [71] where the off-shell helicity amplitudes were constructed using the generalized version of the BCFW recursion for off-shell case and the off-shell gauge invariant amplitudes found were having also the the MHV form.

\subsection{The Ward identity for light-front amplitudes}

In the previous section it has been shown how the recursion relation for the light-front amplitudes is identical with the relation for the matrix elements of straight Wilson lines. The main point is that this recursion involves an off-shell amplitude which is defined to be a gauge invariant extension of the on-shell scattering amplitude. It should be also possible however, to verify the Ward identities directly from the light-front diagrams, without resorting to the approach which involves Wilson lines. It turns out that the evaluation of the Ward identities on the light-front requires an important modification of the rules for the perturbation theory. More details together with the derivation of the modified rules can be found in [33].

In order to illustrate the issue in the light-front, we shall first verify the identity for the lowest order amplitude $(+\rightarrow-++)$. It will become clear that one needs to modify the rules for the computation of the Ward identities, in order to guarantee the four-momentum conservation. As discussed in the first section, the standard light-front rules do not involve the minus components in the calculation, except for the energy denominators, where it is not conserved $[35,38,41]$. This is what gives the off-shell ness of the intermediate states in the light-front diagrams. However, for the Ward identity to hold, the minus components need to be taken care of in the vertices as well. Therefore, the procedure for the computation of this identity needs to be revised. This results in the additional instantaneous-like component in the rules for the light-front perturbation theory in the Ward identity evaluation [33]. After taking this term into account, the result of the calculation is linearly proportional to the energy denominator of the initial state and the Ward identity is satisfied on the light-front when the physical on-shell condition is set.

The Ward identities $[86,87]$ for a generic QCD amplitude $\mathcal{M}$ with external momenta $k_{i}$ on-shell and corresponding polarization vectors $\varepsilon_{i}$, read

$$
\left.\mathcal{M}\left(\epsilon_{1}, \epsilon_{2}, \ldots, \epsilon_{n}\right)\right|_{\varepsilon_{i} \rightarrow k_{i}}=0 \text { for any } i \text {. }
$$

These identities do not work for light-front amplitudes when applied directly and appropriate modifications must be performed to ensure that they are satisfied. Typically, when evaluating the light-front diagrams the minus light cone components are integrated out. This causes the problem for the Ward 
$\left(\mathcal{A}_{1}\right)$

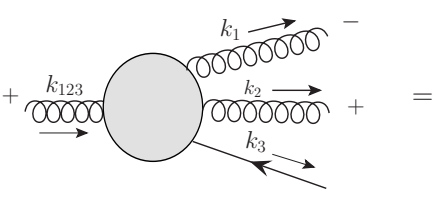

$\left(\mathcal{A}_{3}\right)$

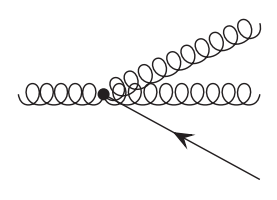

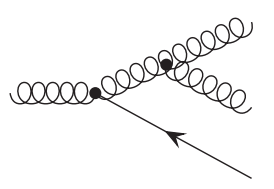

$\left(\mathcal{A}_{4}\right)$

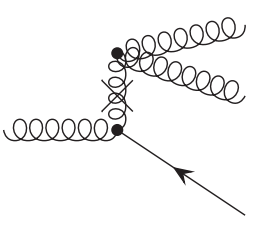

$\left(\mathcal{A}_{2}\right)$

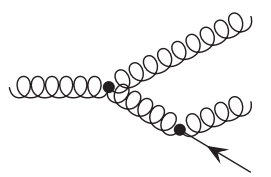

$\left(\mathcal{A}_{5}\right)$

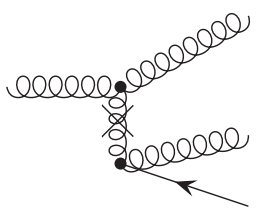

Figure 26: Diagrams for the Ward identity check for $1 \rightarrow 3$ light-front amplitude with helicity configuration $+\rightarrow-+ \pm$. The solid line with an arrow instead of a gluon line represents replacement of a polarization vector with the corresponding momentum. The two right-most diagrams contain standard instantaneous interactions of the light-front theory.

identity since in the latter case a minus light-cone component is inserted into the vertices after that integration when replacing a gluon polarization vector by its momentum.

The problem can be most readily illustrated by the following explicit low-order example. We shall consider here a light-front amplitude for the $1 \rightarrow 3$ process with the helicity configuration $(+\rightarrow-++)$ (first particle is off-shell and its momentum is incoming and the rest are outgoing). This is the lowest non-trivial MHV amplitude, see Sec. 3. We then replace the polarization vector of the third outgoing particle by the corresponding momentum. One can write

$$
\mathcal{M}_{1 \rightarrow 3}^{\left(+\rightarrow-+k_{3}\right)}=\mathcal{A}_{1}+\mathcal{A}_{2}+\mathcal{A}_{3}+\mathcal{A}_{4}+\mathcal{A}_{5}
$$

where $\mathcal{A}_{1}-\mathcal{A}_{5}$ are the contributions from the diagrams depicted in Fig. 26. Here, and below we use a notation for replacement $\epsilon_{i} \leftrightarrow k_{i}$ in the superscript, i.e. we replace the helicity label by the corresponding momentum. Using the rules of the light-front perturbation theory defined in Sec. 2.5 and color-ordered vertices shown in Table 2, adding the contributions from all the diagrams one finally gets the following result for the Ward identity

$$
\begin{aligned}
\mathcal{M}_{1 \rightarrow 3}^{\left(+\rightarrow-+k_{3}\right)}=-i g^{2}\left[2 \frac{z_{3}}{z_{12}}\left(z_{1} v_{1(123)}+z_{2} v_{13}\right)+\left(z_{23}+z_{2}\right)\right. & v_{(123) 1} \\
& \left.-\frac{z_{2} z_{3} z_{123}}{z_{1} z_{12}^{2} v_{12}^{*}}\left(z_{123}+z_{12}\right) v_{(123) 3} v_{(123) 3}^{*}\right] .
\end{aligned}
$$

The above result is obtained without the full four-momentum conservation. This is because, according to light-front perturbation theory rules, at each vertex there is a Dirac delta only for the plus and transverse components. There is no conservation of the minus components, which for each momentum are fixed by the on-shell condition. If the Ward identity was satisfied, the result above (260) should be proportional to the energy denominator $D_{(1,2,3)}$, which vanishes for physical on-shell partons. However this is not the case for (260) and this expression does not vanish when taken on-shell, i.e. $D_{(1,2,3)} \rightarrow 0$.

The above problem stems from the fact that the light-front diagrams in Fig. 26 were obtained assuming that there are no external minus components. Indeed, for an amplitude calculation, the polarization vectors (53) project only on plus and transverse components. This is evident from the form of the vertices in the light-front, which are listed in Table 2. The only minus components that flow inside diagrams were integrated out giving energy denominators and the instantaneous terms. However, 
for the above Ward identity check, the triple gluon vertex which appears, for example, in $\mathcal{A}_{1}$, reads

$$
\begin{aligned}
V_{3}^{+\rightarrow+k_{3}}\left(-k_{23}, k_{2}, k_{3}\right)=i g\left[-k_{3} \cdot\left(k_{2}+\right.\right. & \left.k_{23}\right)\left(\varepsilon_{23}^{+}\right)^{*} \cdot \varepsilon_{2}^{+}+\left(\varepsilon_{23}^{+}\right)^{*} \cdot\left(k_{2}-k_{3}\right) k_{3} \cdot \varepsilon_{2}^{+} \\
& \left.+\varepsilon_{2}^{+} \cdot\left(k_{23}+k_{3}\right)\left(\varepsilon_{23}^{+}\right)^{*} \cdot k_{3}\right]=i g \frac{z_{2} z_{3}\left(z_{23}+z_{2}\right)}{z_{23}} v_{23} v_{23}^{*} .
\end{aligned}
$$

The problematic term is the first one in the square bracket, since it contains combinations of the momenta which have minus components. Formally, we cannot write $k_{3} \cdot\left(k_{2}+k_{23}\right)=2 k_{2} \cdot k_{3}$ since we do not have full momentum conservation. We have to consider $k_{2} \cdot k_{3}$ and $k_{23} \cdot k_{3}$ as different scalar products and this causes the Ward identity to fail.

As already mentioned in the beginning of this section, in the correct procedure one should integrate out all the minus components. In (261) the internal minus component $k_{23}^{-}$appears in the numerator and thus leads to the additional instantaneous-like additional contribution to (259) (in addition to the terms indicated in Fig. 26). An explicit calculation of this term was performed in [33]. It turns out, that this additional term added to (261) gives

$$
\tilde{V}_{3}^{+\rightarrow+k_{3}}\left(-k_{23}, k_{2}, k_{3}\right)=2 i g z_{2} z_{3} v_{23} v_{23}^{*},
$$

and it effectively restores the full momentum conservation (in the numerator). Collecting all the terms one arrives at the following result

$$
\mathcal{M}_{1 \rightarrow 3}^{\left(+\rightarrow-+k_{3}\right)}=i g^{2} \frac{z_{2}}{z_{1}} \frac{D_{(1,2,3)}}{v_{12}^{*}}
$$

which obviously vanishes in the case of the on-shell amplitude, i.e. for $D_{(1,2,3)} \rightarrow 0$. Therefore after this modification the Ward identity on the light-front is satisfied as it should be.

Let us now come back to the recursion relation (230). In that relation the new amplitude $M$ that actually solves the recurrence, has exactly the form of the MHV amplitude. Therefore, once we impose the on-shell condition for the process, the result is equal to the MHV amplitude as expected. The amplitude $M$, however, as it stands in the recursion relation, is an off-shell object. It turns out however, that it has a remarkable property, namely it is gauge invariant, as it was demonstrated in the previous section using the Wilson line approach.

This can be also explicitly illustrated by taking (235) for $n=3$ and checking the Ward identity. Of course, one needs to take care of the issues discussed above and include the new instantaneous terms. The Ward identity check using the recursion gives

$$
M_{1 \rightarrow 3}^{\left(+\rightarrow-+k_{3}\right)}=\mathcal{M}_{1 \rightarrow 3}^{\left(+\rightarrow-+k_{3}\right)}-\frac{D_{(1,2,3)}}{D_{(12,3)}} \mathcal{M}_{1 \rightarrow 2}^{\left(+\rightarrow+k_{3}\right)} \frac{i}{z_{12} D_{(1,2)}} M_{1 \rightarrow 2}^{(+\rightarrow-+)}
$$

In the above expression we have replaced the polarization vector both in the $1 \rightarrow 3$ amplitude and $1 \rightarrow 2$ subamplitude. The second term can be simplified to

$$
\frac{D_{(1,2,3)}}{D_{(12,3)}}\left(2 i g z_{3} z_{123} v_{(123) 3} v_{(123) 3}^{*}\right) \frac{i}{z_{12} D_{(1,2)}}\left(2 i g z_{2} v_{(12) 1}\right)=i g^{2} \frac{z_{2}}{z_{1}} \frac{D_{(1,2,3)}}{v_{12}^{*}}
$$

where we have used the relations $z_{12} D_{(1,2)}=2 z_{1} z_{2} v_{12} v_{12}^{*}, z_{12} D_{(12,3)}=2 z_{123} z_{3} v_{(123) 3} v_{(123) 3}^{*}$ and $z_{1} v_{(12) 1}=$ $-z_{2} v_{12}$. We see that (265) precisely cancels the previously derived term (263), leaving $M_{1 \rightarrow 3}^{\left(+\rightarrow-+k_{3}\right)}$ equal to zero. Therefore $M_{1 \rightarrow 3}^{(+\rightarrow-++)}$ is the gauge invariant amplitude irrespectively whether the incoming leg is on-shell or off-shell. 
Using the results from the recursion relations one can thus conclude that the Ward identity for $M$ is satisfied in general, for arbitrary number of external legs

$$
M_{1 \rightarrow N}^{\left(+\rightarrow-+\ldots k_{i} \cdots+\right)}=0
$$

Thus the recursion (235) which has been proven entirely from the light-front diagrams, using algebraic methods, arises as a consequence of the gauge invariance.

\section{Conclusions}

In this review we have discussed the computational methods of different objects, like fragmentation functions, wave functions and scattering amplitudes, using the formalism of the light-front perturbation theory. The light-front formalism offers a lot of unique advantages, for example in terms of the simplicity of the vacuum and thus can be used to define the partonic content of the hadron by means of the Fock expansion. Part of this simplicity stems from the equivalence of the subgroup of the Poincare group to the 2-dimensional Galilean group of transformations when using the light-front formalism.

When computing the amplitudes there are number of differences between the standard formulation and the light-front formulation. Unlike the standard framework, which is covariant, the light-front theory is not explicitly covariant when computing partial results. One needs to sum over the different light-front times orderings of the vertices in the graphs, and this restores the covariance of the final result. The spinor variables which are so useful in the computation of the scattering amplitudes in the helicity formalism emerge naturally in the context of the light-front theory. They are related to the 'velocities' of the particles on the light front and are manifestation of the Galilean group as mentioned above. Working with off-shell amplitudes is relatively straightforward on the light-front. Since the particles are always on-shell on the light-front, the off-shellness comes from the energy denominators which are the differences in the minus components (the non-conservation of the minus-components). The on-shell object can be then restored by imposing the physical condition of the conservation of this component between the final and initial states. When computing the helicity amplitudes the standard formalism offers a little bit more flexibility due to the fact that one has the choice of the reference momenta for the polarization vectors. By a clever choice one can eliminate large set of the diagrams, simplifying the results. On the light-front the reference momentum is chosen to be equal to one particular light-like vector, the same for all particles and so this flexibility is lost.

Light-front formalism offers more physical interpretation in different contexts. For example recently [88], it has been demonstrated that in the equations which govern high energy evolution of the gluon density, the important corrections which stem from kinematics can be easily understood from the light-front perspective. Namely, such equations resum the subsequent gluon emissions, which in the light-front perturbation theory should be ordered in the light-front time. When this requirement is taken into account, the kinematical constraints follow naturally in this formalism.

We showed some very intriguing properties which arise on the light-front, for example the fact that there exists a new recurrence relation for the off-shell amplitudes which includes the gauge invariant amplitudes. Remarkably this recurrence relation can be shown to be identical to the recurrence relation which involves infinite straight Wilson lines. It certainly points to the fundamental importance of the gauge invariance property in any QCD applications.

For the future outlook, it would certainly be interesting to investigate whether some of these lightfront recursion relations can be extended for more complicated helicity configurations than considered so far. Recent work using the Wilson line framework, indicates that this is indeed possible, that is one can still find the similar relation which involves the gauge invariant off-shell amplitudes. Another possible extension is to compute the off-shell amplitudes which include quarks. Recurrence relations based on 
the factorization properties can be also constructed in the case of the fermions without fundamental complications.

In the present review we have limited the discussion of the amplitudes to the tree level case. As indicated in the introduction there has been an enormous progress to compute the on-shell amplitudes with loops which are relevant for the phenomenology. The off-shell amplitudes at the tree level which were discussed here, are nevertheless very important for the phenomenology as they can be used as part of the framework with the unintegrated parton distribution functions. This approach treats the kinematics of the final state much more accurately than the framework based on the on-shell amplitudes and the collinear factorization and can be important when considering exclusive final states in the hadronic collisions. Nevertheless, it would be certainly interesting and important to compute the higher order corrections to the off-shell amplitudes to estimate the relevance of loops and the convergence of the perturbative series. As an important step it would be interesting to explore whether the recurrence relations with off-shell amplitudes can be constructed and then used to efficiently compute the loop diagrams on the light-front. Another worthwhile line of investigation is to understand better the duality between the momentum space fragmentation functions and the wave-functions and the relations to the Wilson lines in both cases. In any case the light-front methods continue to provide a unique insight into the intricacies of the strong interactions and quantum field theory in general.

\section{Acknowledgments}

This work was supported in part by the DOE grant No. DE-SC-0002145, grant No. DE-FG0293ER40771 and by the Polish NCN grant DEC-2011/01/B/ST2/03915. Diagrams have been prepared with Jaxodraw [89].

\section{References}

[1] P. A. Dirac, "Forms of Relativistic Dynamics," Rev.Mod.Phys., vol. 21, pp. 392-399, 1949.

[2] H. Leutwyler and J. Stern, "Relativistic Dynamics on a Null Plane," Annals Phys., vol. 112, p. 94, 1978.

[3] L. Susskind, "Model of selfinduced strong interactions," Phys.Rev., vol. 165, pp. 1535-1546, 1968.

[4] J. B. Kogut and L. Susskind, "The Parton picture of elementary particles," Phys.Rept., vol. 8, pp. $75-172,1973$.

[5] A. Casher and L. Susskind, "Chiral magnetism (or magnetohadrochironics)," Phys.Rev., vol. D9, pp. 436-460, 1974.

[6] M. Burkardt, "Much ado about nothing: Vacuum and renormalization on the light front," hep$\mathrm{ph} / 9709421,1997$.

[7] M. Burkardt, "Much ado about nothing: Vacuum and renormalization in the light front framework," Nucl.Phys., vol. A670, pp. 72-75, 2000.

[8] T. Heinzl, "Light cone quantization: Foundations and applications," Lect.Notes Phys., vol. 572, pp. 55-142, 2001.

[9] S. Weinberg, "Dynamics at infinite momentum," Phys.Rev., vol. 150, pp. 1313-1318, 1966.

[10] K. Yamawaki, "Zero mode problem on the light front," 1998. 
[11] P. P. Srivastava and S. J. Brodsky, "A Unitary and renormalizable theory of the standard model in ghost free light cone gauge," Phys.Rev., vol. D66, p. 045019, 2002.

[12] S. R. Beane, "Broken Chiral Symmetry on a Null Plane," Annals Phys., vol. 337, pp. 111-142, 2013.

[13] S. J. Brodsky and R. Shrock, "On Condensates in Strongly Coupled Gauge Theories," Proc.Nat.Acad.Sci., vol. 108, pp. 45-50, 2011.

[14] S. J. Brodsky and R. Shrock, "Standard-Model Condensates and the Cosmological Constant," Proc.Nat.Acad.Sci., vol. 108, pp. 45-50, 2011.

[15] S. J. Brodsky, C. D. Roberts, R. Shrock, and P. C. Tandy, "Essence of the vacuum quark condensate," Phys.Rev., vol. C82, p. 022201, 2010.

[16] S. J. Brodsky, C. D. Roberts, R. Shrock, and P. C. Tandy, "Confinement contains condensates," Phys.Rev., vol. C85, p. 065202, 2012.

[17] S. J. Brodsky and G. F. de Teramond, "Light front hadron dynamics and AdS / CFT correspondence," Phys.Lett., vol. B582, pp. 211-221, 2004.

[18] A. H. Mueller, "Soft gluons in the infinite momentum wave function and the BFKL pomeron," Nucl.Phys., vol. B415, pp. 373-385, 1994.

[19] S. J. Parke and T. Taylor, "An Amplitude for $n$ Gluon Scattering," Phys.Rev.Lett., vol. 56, p. 2459, 1986.

[20] F. A. Berends and W. Giele, "Recursive Calculations for Processes with n Gluons," Nucl.Phys., vol. B306, p. 759, 1988.

[21] R. Britto, F. Cachazo, and B. Feng, "New recursion relations for tree amplitudes of gluons," Nucl.Phys., vol. B715, pp. 499-522, 2005.

[22] R. Britto, F. Cachazo, B. Feng, and E. Witten, "Direct proof of tree-level recursion relation in Yang-Mills theory," Phys.Rev.Lett., vol. 94, p. 181602, 2005.

[23] M. L. Mangano, M. Moretti, F. Piccinini, R. Pittau, and A. D. Polosa, "ALPGEN, a generator for hard multiparton processes in hadronic collisions," JHEP, vol. 0307, p. 001, 2003.

[24] T. Gleisberg and S. Hoeche, "Comix, a new matrix element generator," JHEP, vol. 0812, p. 039, 2008.

[25] R. Kleiss and G. van den Oord, "CAMORRA: a C++ library for recursive computation of particle scattering amplitudes," Comput.Phys.Commun., vol. 182, pp. 435-447, 2011.

[26] A. Cafarella, C. G. Papadopoulos, and M. Worek, "Helac-Phegas: A Generator for all parton level processes," Comput.Phys.Commun., vol. 180, pp. 1941-1955, 2009.

[27] L. J. Dixon, J. M. Henn, J. Plefka, and T. Schuster, "All tree-level amplitudes in massless QCD," JHEP, vol. 1101, p. 035, 2011.

[28] J. Alwall, M. Herquet, F. Maltoni, O. Mattelaer, and T. Stelzer, "MadGraph 5 : Going Beyond," JHEP, vol. 1106, p. 128, 2011. 
[29] L. Motyka and A. M. Stasto, "Exact kinematics in the small x evolution of the color dipole and gluon cascade," Phys.Rev., vol. D79, p. 085016, 2009.

[30] C. Cruz-Santiago and A. Stasto, "Gluon cascades and amplitudes in light-front perturbation theory," Nucl.Phys., vol. B869, pp. 1-30, 2013.

[31] S. J. Brodsky and C.-R. Ji, "Factorization Property of the Deuteron," Phys.Rev., vol. D33, p. 2653, 1986.

[32] C. Cruz-Santiago and A. Stasto, "Recursion relations and scattering amplitudes in the light-front formalism," Nucl.Phys., vol. B875, pp. 368-387, 2013.

[33] C. Cruz-Santiago, P. Kotko, and A. Stasto, "Recursion relations for multi-gluon off-shell amplitudes on the light-front and Wilson lines," Nucl.Phys., vol. B895, pp. 132-160, 2015.

[34] P. Kotko, "Wilson lines and gauge invariant off-shell amplitudes," JHEP, vol. 1407, p. 128, 2014.

[35] J. B. Kogut and D. E. Soper, "Quantum Electrodynamics in the Infinite Momentum Frame," Phys.Rev., vol. D1, pp. 2901-2913, 1970.

[36] J. Bjorken, J. B. Kogut, and D. E. Soper, "Quantum Electrodynamics at Infinite Momentum: Scattering from an External Field," Phys.Rev., vol. D3, p. 1382, 1971.

[37] S. J. Brodsky and G. P. Lepage, "Exclusive Processes in Quantum Chromodynamics," Adv.Ser.Direct.High Energy Phys., vol. 5, pp. 93-240, 1989.

[38] S. J. Brodsky, H.-C. Pauli, and S. S. Pinsky, "Quantum chromodynamics and other field theories on the light cone," Phys.Rept., vol. 301, pp. 299-486, 1998.

[39] G. P. Lepage, S. J. Brodsky, T. Huang, and P. Mackenzie, "HADRONIC WAVE FUNCTIONS IN QCD," pp. 83-141, 1982.

[40] R. Venugopalan, "Introduction to light cone field theory and high-energy scattering," Lect.Notes Phys., vol. 516, p. 89, 1999.

[41] G. P. Lepage and S. J. Brodsky, "Exclusive Processes in Perturbative Quantum Chromodynamics," Phys.Rev., vol. D22, p. 2157, 1980.

[42] R. Gastmans and T. Wu, "The Ubiquitous photon: Helicity method for QED and QCD," Int.Ser.Monogr.Phys., vol. 80, pp. 1-648, 1990.

[43] M. L. Mangano and S. J. Parke, "Multiparton amplitudes in gauge theories," Phys.Rept., vol. 200, pp. 301-367, 1991.

[44] L. J. Dixon, "Calculating scattering amplitudes efficiently," hep-ph/9601359, 1996.

[45] L. J. Dixon, "A brief introduction to modern amplitude methods," arXiv 1310.5353, 2014.

[46] Z. Bern, L. J. Dixon, and D. A. Kosower, "On-Shell Methods in Perturbative QCD," Annals Phys., vol. 322, pp. 1587-1634, 2007.

[47] R. Britto, "Loop Amplitudes in Gauge Theories: Modern Analytic Approaches," J.Phys., vol. A44, p. $454006,2011$.

[48] H. Ita, "Susy Theories and QCD: Numerical Approaches," J.Phys., vol. A44, p. 454005, 2011. 
[49] R. K. Ellis, Z. Kunszt, K. Melnikov, and G. Zanderighi, "One-loop calculations in quantum field theory: from Feynman diagrams to unitarity cuts," Phys.Rept., vol. 518, pp. 141-250, 2012.

[50] L. F. Alday and R. Roiban, "Scattering Amplitudes, Wilson Loops and the String/Gauge Theory Correspondence," Phys.Rept., vol. 468, pp. 153-211, 2008.

[51] J. Drummond, "Hidden Simplicity of Gauge Theory Amplitudes," Class.Quant.Grav., vol. 27, p. 214001, 2010.

[52] J. Bjorken and M. Chen, "HIGH-ENERGY TRIDENT PRODUCTION WITH DEFINITE HELICITIES," Phys.Rev., vol. 154, pp. 1335-1337, 1966.

[53] G. R. Henry, "Trident Production with Nuclear Targets," Phys.Rev., vol. 154, pp. 1534-1536, 1967.

[54] P. De Causmaecker, R. Gastmans, W. Troost, and T. T. Wu, "Helicity Amplitudes for Massless QED," Phys.Lett., vol. B105, p. 215, 1981.

[55] P. De Causmaecker, R. Gastmans, W. Troost, and T. T. Wu, "Multiple Bremsstrahlung in Gauge Theories at High-Energies. 1. General Formalism for Quantum Electrodynamics," Nucl.Phys., vol. B206, p. 53, 1982.

[56] F. A. Berends, R. Kleiss, P. De Causmaecker, R. Gastmans, W. Troost, et al., "Multiple Bremsstrahlung in Gauge Theories at High-Energies. 2. Single Bremsstrahlung," Nucl.Phys., vol. B206, p. 61, 1982.

[57] D. A. Kosower, "Light Cone Recurrence Relations for QCD Amplitudes," Nucl.Phys., vol. B335, p. 23, 1990.

[58] F. A. Berends and W. Giele, "The Six Gluon Process as an Example of Weyl-Van Der Waerden Spinor Calculus," Nucl.Phys., vol. B294, p. 700, 1987.

[59] M. L. Mangano, S. J. Parke, and Z. Xu, "Duality and Multi - Gluon Scattering," Nucl.Phys., vol. B298, p. 653, 1988.

[60] M. L. Mangano, S. J. Parke, and Z. Xu, "Dual Amplitudes and Multi - Gluon Processes," 1987.

[61] M. L. Mangano, "The Color Structure of Gluon Emission," Nucl.Phys., vol. B309, p. 461, 1988.

[62] P. Cvitanovic, P. Lauwers, and P. Scharbach, "Gauge Invariance Structure of Quantum Chromodynamics," Nucl.Phys., vol. B186, p. 165, 1981.

[63] Z. Bern and D. A. Kosower, "Color decomposition of one loop amplitudes in gauge theories," Nucl.Phys., vol. B362, pp. 389-448, 1991.

[64] G. 't Hooft, "A Planar Diagram Theory for Strong Interactions," Nucl.Phys., vol. B72, p. 461, 1974.

[65] G. 't Hooft, "A Two-Dimensional Model for Mesons," Nucl.Phys., vol. B75, p. 461, 1974.

[66] M. T. Grisaru, H. Pendleton, and P. van Nieuwenhuizen, "Supergravity and the S Matrix," Phys.Rev., vol. D15, p. 996, 1977.

[67] M. T. Grisaru and H. Pendleton, "Some Properties of Scattering Amplitudes in Supersymmetric Theories," Nucl.Phys., vol. B124, p. 81, 1977. 
[68] B. Feng and M. Luo, "An Introduction to On-shell Recursion Relations," Front.Phys., vol. 7, pp. 533-575, 2012.

[69] B. Truijen, "Britto-Cachazo-Feng-Witten Recursion: An Introduction," master thesis.

[70] N. Arkani-Hamed and J. Kaplan, "On Tree Amplitudes in Gauge Theory and Gravity," JHEP, vol. 0804, p. 076, 2008.

[71] A. van Hameren, "BCFW recursion for off-shell gluons," JHEP, vol. 1407, p. 138, 2014.

[72] M. Bury and A. van Hameren, "Numerical evaluation of multi-gluon amplitudes for High Energy Factorization," arXiv 1503.08612, 2015.

[73] A. van Hameren and M. Serino, "BCFW recursion for TMD parton scattering," arXiv 1504.00315, 2015.

[74] S. J. Brodsky and B. Chertok, "The Asymptotic Form-Factors of Hadrons and Nuclei and the Continuity of Particle and Nuclear Dynamics," Phys.Rev., vol. D14, pp. 3003-3020, 1976.

[75] S. J. Brodsky and B. Chertok, "The Deuteron Form-Factor and the Short Distance Behavior of the Nuclear Force," Phys.Rev.Lett., vol. 37, p. 269, 1976.

[76] C. A. Cruz-Santiago, "Gluon Wavefunctions and Amplitudes on the Light-Front," Acta Phys.Polon.Supp., vol. 6, pp. 201-206, 2013.

[77] S. J. Brodsky and G. F. de Teramond, "QCD and Light-Front Dynamics," PoS, vol. LC2010, p. 070, 2010.

[78] S. J. Brodsky, D. S. Hwang, B.-Q. Ma, and I. Schmidt, "Light cone representation of the spin and orbital angular momentum of relativistic composite systems," Nucl.Phys., vol. B593, pp. 311-335, 2001.

[79] A. Harindranath and R. Kundu, "On Orbital angular momentum in deep inelastic scattering," Phys.Rev., vol. D59, p. 116013, 1999.

[80] B.-Q. Ma and I. Schmidt, "The Quark orbital angular momentum in a light cone representation," Phys.Rev., vol. D58, p. 096008, 1998.

[81] F. Cachazo, P. Svrcek, and E. Witten, "MHV vertices and tree amplitudes in gauge theory," JHEP, vol. 0409, p. 006, 2004.

[82] A. van Hameren, P. Kotko, and K. Kutak, "Multi-gluon helicity amplitudes with one off-shell leg within high energy factorization," JHEP, vol. 1212, p. 029, 2012.

[83] A. van Hameren, P. Kotko, and K. Kutak, "Helicity amplitudes for high-energy scattering," JHEP, vol. 1301, p. 078, 2013.

[84] L. Lipatov, "Gauge invariant effective action for high-energy processes in QCD," Nucl.Phys., vol. B452, pp. 369-400, 1995.

[85] E. Antonov, L. Lipatov, E. Kuraev, and I. Cherednikov, "Feynman rules for effective Regge action," Nucl.Phys., vol. B721, pp. 111-135, 2005.

[86] J. C. Ward, "An Identity in Quantum Electrodynamics," Phys.Rev., vol. 78, p. 182, 1950. 
[87] Y. Takahashi, "On the generalized Ward identity," Nuovo Cim., vol. 6, p. 371, 1957.

[88] E. Iancu, J. Madrigal, A. Mueller, G. Soyez, and D. Triantafyllopoulos, "Resumming double logarithms in the QCD evolution of color dipoles," Phys.Lett., vol. B744, pp. 293-302, 2015.

[89] D. Binosi and L. Theussl, "JaxoDraw: A Graphical user interface for drawing Feynman diagrams," Comput.Phys.Commun., vol. 161, pp. 76-86, 2004. 